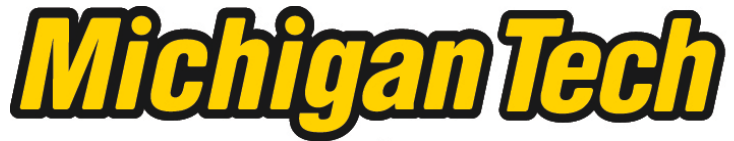 \\ Michigan Technological University Create the Future Digital Commons @ Michigan Tech
}

2014

\section{Thickness measurement of dynamic thin liquid films generated by plug-annular flow in non-wetting microchannels}

David C. Deisenroth

Michigan Technological University

Follow this and additional works at: https://digitalcommons.mtu.edu/etds

Part of the Mechanical Engineering Commons

Copyright 2014 David C. Deisenroth

\section{Recommended Citation}

Deisenroth, David C., "Thickness measurement of dynamic thin liquid films generated by plug-annular flow in non-wetting microchannels", Master's Thesis, Michigan Technological University, 2014.

https://doi.org/10.37099/mtu.dc.etds/772

Follow this and additional works at: https://digitalcommons.mtu.edu/etds

Part of the Mechanical Engineering Commons 
THICKNESS MEASUREMENT OF DYNAMIC THIN LIQUID FILMS GENERATED BY PLUG-ANNULAR FLOW IN NON-WETTING MICROCHANNELS

\author{
By
}

David C. Deisenroth

\begin{abstract}
A THESIS
Submitted in partial fulfillment of the requirements for the degree of MASTER OF SCIENCE

In Mechanical Engineering

MICHIGAN TECHNOLOGICAL UNIVERSITY

2014
\end{abstract}

Copyright (C) 2014 David C. Deisenroth 

This thesis has been approved in partial fulfillment of the requirements for the Degree of MASTER OF SCIENCE in Mechanical Engineering.

Department of Mechanical Engineering - Engineering Mechanics

Dissertation Advisor: Dr. Jeffrey S. Allen

Committee Member: Dr. Chang Kyoung Choi

Committee Member: Dr. Adrienne Minerick

Department Chair: Professor William W. Predebon 



\section{CONTENTS}

Table of Contents . . . . . . . . . . . . . . . . . . . . v v

List of Figures . . . . . . . . . . . . . . . . . . . . . . . . . . . . . . viii

Acknowledgments ....................... ix

Nomenclature ........................... xii

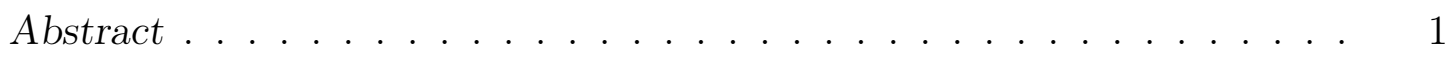

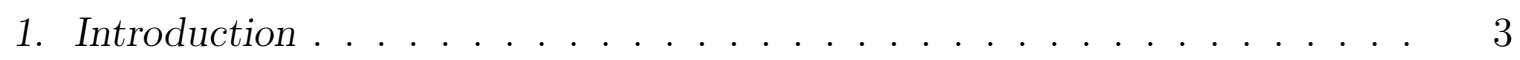

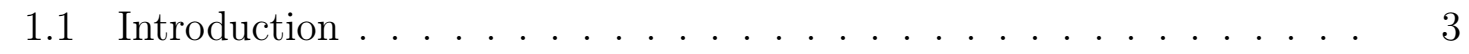

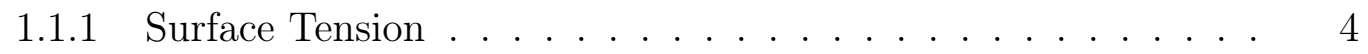

1.1 .2 Wettability . . . . . . . . . . . . . . . . . 4

$1.1 .3 \quad$ Contact Angle . . . . . . . . . . . . . . . . . . . . . . . . 5

1.1 .4 Scaling Parameters . . . . . . . . . . . . . . . . . . . . 6

1.1.5 Two-Phase Flow Regimes . . . . . . . . . . . . . . . . . . . . . . . 8

1.2 Two-Phase Flow Research . . . . . . . . . . . . . . . . . . . . . . . . 11

1.2.1 Liquid Film Deposition Behind a Receding Meniscus . . . . . 11

1.2.1.1 Wetting Film . . . . . . . . . . . . . . . . 11

$1.2 .1 .2 \quad$ Non-wetting Film . . . . . . . . . . . . . . . . . . . . 13

1.2 .2 Plug Flow . . . . . . . . . . . . . . . . . . . . . . . . . . . . . . . . . . . . . . . . . . . . .

1.2 .3 Plug-Annular . . . . . . . . . . . . . . . . . . . . . . . . . . . . . . . . . . . . . . . . 22

1.2.4 Annular . . . . . . . . . . . . . . . . . . . . . . 23

1.3 Summary . . . . . . . . . . . . . . . . . . . 26

2. Scope and Goal . . . . . . . . . . . . . . . . . . . . . . . . . 27

3. Liquid Film Thickness Measurement . . . . . . . . . . . . . . . . . . . . . 37

3.1 Current Research . . . . . . . . . . . . . . . . . . . . . . 37

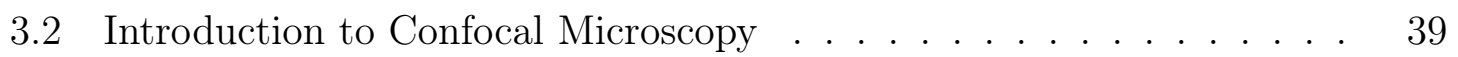

3.2.1 Optical Slice . . . . . . . . . . . . . . . . . . . . 41 
3.2 .2 Cross View-Field Intensity . . . . . . . . . . . . . . . . . . . . 43

3.2 .3 Thickness Measurement of a Known Medium. . . . . . . . . . 44

3.3 Experimental Setup . . . . . . . . . . . . . . . . . . . . . . . . . 48

3.3 .1 Test Section . . . . . . . . . . . . . . . . . . . . . . . . 49

3.3 .2 Imaging . . . . . . . . . . . . . . . . . . 51

3.3.2.1 Macro-View . . . . . . . . . . . . . . . 51

3.3.2.2 Confocal View . . . . . . . . . . . . . . . . . . 51

3.3.3 Experimental Procedure . . . . . . . . . . . . . . . . 52

3.4 Image Processing . . . . . . . . . . . . . . . . . . . . 53

3.5 Focus Depth Correction . . . . . . . . . . . . . . . . . . . . . . . 59

3.6 Uncertainty . . . . . . . . . . . . . . . . . . . . . 61

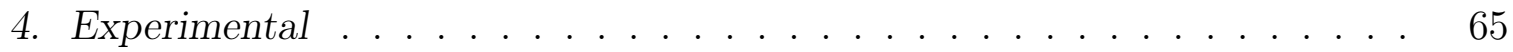

4.1 Test Section Properties . . . . . . . . . . . . . . . . . . . 65

4.1 .1 Refractive Index . . . . . . . . . . . . . . . . . . . 65

4.1 .2 Contact Angle and Surface Properties . . . . . . . . . . . . . . 66

4.2 Initial Observations . . . . . . . . . . . . . . . . . 66

$4.3 \quad$ Film Thickness Measurement Experimental Results . . . . . . . . . . 72

5. Conclusions . . . . . . . . . . . . . . . . . . . . . . . . . 79

References . . . . . . . . . . . . . . . . . . 88

\begin{tabular}{ll}
\hline Appendices & 89
\end{tabular}

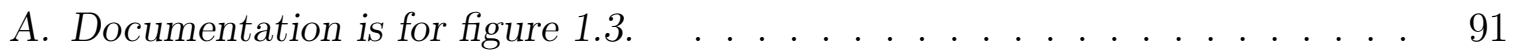

B. PrairieView Correspondence . . . . . . . . . . . . . . . . . . . . . 93

C. Intensity Data . . . . . . . . . . . . . . . . . . . . . . . . . . . . . . . 99 


\section{List OF FIGURES}

1.1 Illustration of apparent contact angle . . . . . . . . . . . . . . . . . 6

1.2 Illustration of flow regimes . . . . . . . . . . . . . . . . . . . . . . . . 9

1.3 Two-phase flow regimes . . . . . . . . . . . . . . . . . . . . . . . . . 10

1.4 Illustration of the Landau-Levich problem. . . . . . . . . . . . . . . . 12

1.5 Illustration of a bubble moving through a capillary tube. . . . . . . . 17

$2.1 \quad$ Plug-annular flow in a non-wetting microchannel . . . . . . . . . . . . 28

2.2 Shock structure formation in plug-annular regime . . . . . . . . . . . 29

2.3 Non-wetting film morphology . . . . . . . . . . . . . . . . . . . . . . 31

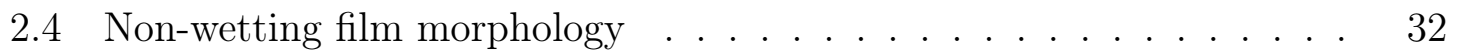

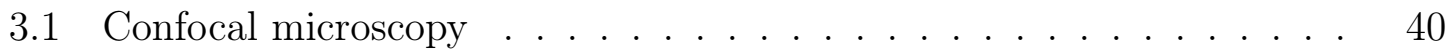

3.2 Optical slice thickness . . . . . . . . . . . . . . . . . . . . . . . . . . . . . . . . . . . .

3.3 Cross view-field intensity . . . . . . . . . . . . . . . . . . . . . . . 44

3.4 Cross view-field intensity along the x-axis . . . . . . . . . . . . . . . . . 45

3.5 Cross view-field intensity along the y-axis . . . . . . . . . . . . . . 46

3.6 Intensity profile of a cover slip . . . . . . . . . . . . . . . . . . . . . . . . . . 47

3.7 An overview of the experimental setup . . . . . . . . . . . . . . . . . 49

3.8 Test section construction . . . . . . . . . . . . . . . . 50

3.9 Location of plug-annular features . . . . . . . . . . . . . . . . . . . . . . . . . . . 54

3.10 The thick and thin film thicknesses . . . . . . . . . . . . . . . . . 55

3.11 Intensity profiles at multiple optical slice locations . . . . . . . . . . . 56

3.12 Location of plug-annular features . . . . . . . . . . . . . . . . . . . . . . 57

3.13 Intensity profile . . . . . . . . . . . . . . . . . . . . . 58

3.14 Beam path into the test section . . . . . . . . . . . . . . 60

3.15 Illustration of bit depth uncertainty . . . . . . . . . . . . . . 63

4.1 No shock is observed . . . . . . . . . . . . . . . . . . . . 67

4.2 Shock structure formation . . . . . . . . . . . . . . . . . . . . . . . . . . . 69

$4.3 \quad$ Film rupture in a non-wetting Taylor bubble . . . . . . . . . . . . . . 70

4.4 Location of shock rapidly changes after pinch off . . . . . . . . . . . . 71 
4.5 Film morphology becomes more dynamic and unstable . . . . . . . . 72

$4.6 \quad$ Reflection intensity of channel wall-air interface . . . . . . . . . . . . 73

4.7 Reflection intensity of thin film-air interface . . . . . . . . . . . . . . 75

4.8 Lorentzian fit of reflection intensity . . . . . . . . . . . . . . . . . . . 75

4.9 Reflection intensity of thick film-air interface . . . . . . . . . . . . . 76

4.10 Lorentzian fit of reflection intensity . . . . . . . . . . . . . . . . 76

4.11 Film thickness as a function of Capillary number . . . . . . . . . . . 77

3.1 Reflection intensity of channel wall-air interface . . . . . . . . . . . . 100

3.2 Lorentzian fit of reflection intensity . . . . . . . . . . . . . . . . . 100

3.3 Lorentzian fit of reflection intensity . . . . . . . . . . . . . . . . . . . 101

3.4 Reflection intensity of channel wall-air interface . . . . . . . . . . . . 101

3.5 Lorentzian fit of reflection intensity . . . . . . . . . . . . . . . . . . 102 


\section{ACKNOWLEDGMENTS}

I would like to sincerely thank Dr. Jeff Allen for his exceptional advising during the course of this accelerated Master's degree - helping with everything from technical challenges in research, to career and proposal writing advice, and support in organizing international collaborations. I would also like to thank Joe Hernandez for his experimental collaboration and support in using equipment necessary for this research. Thank you to all the members of the Microfluidics and Interfacial Transport Lab, my friends, and family for your support and encouragement. 



\section{NomenClature}

\begin{tabular}{ll}
$a$ & molecular size \\
$A$ & surface area \\
$A_{L L}^{[6]}$ & liquid/liquid Hamaker constant \\
$A_{M}$ & exposed area of a molecule \\
$A_{S L}^{[6]}$ & solid/liquid Hamaker constant \\
$B o$ & Bond number \\
$c^{*}$ & critical wave front velocity \\
$C a$ & Capillary number \\
$d_{B C}$ & length of dynamical meniscus \\
$D$ & self-diffusion coefficient \\
$E$ & molecular cohesion energy \\
$g$ & acceleration due to gravity \\
$G_{6}(\theta)$ & an angular correction function \\
$h$ & film thickness \\
$h_{\infty}$ & film thickness as $y \rightarrow \infty$ \\
$h_{f}$ & thickness of thick film region \\
$h_{L L}$ & Landau-Levich film thickness \\
$h_{m}$ & characteristic molecular thickness \\
$h_{n o n w e t t i n g}$ & height of non-wetting (thick) film \\
$h_{r}$ & thickness of flat film region \\
$\hat{H}$ & film thickness non-dimensionalized by $l_{c}$ \\
$\mathbf{I}$ & identity matrix \\
$k$ & light ray angle of incidence \\
$k_{B} T$ & measure of molecular thermal energy \\
$l$ & characteristic length of meniscus, or radius \\
$l_{s}$ & slip length \\
$l_{c}$ & capillary length \\
$n$ & refractive index \\
$\mathbf{n}$ & normal unit vector \\
$p$ & pressure \\
$P_{r}$ & fraction of light reflected \\
$P_{t}$ & fraction of light transmitted \\
& \\
& \\
\hline &
\end{tabular}


$q \quad$ liquid flux

$Q \quad$ liquid flux parameter

$r_{b} \quad$ beam radius

$r_{c} \quad$ channel radius

$r_{f} \quad$ film interior radius

$r_{w} \quad$ radius of beam waist

$R \quad$ radius of curvature

$S \quad$ spreading parameter of a solid-fluid-fluid group

$\mathrm{T}$ dimensionless velocity gradient matrix

$T_{n} \quad$ normal stress on bubble surface

$T_{t} \quad$ tangential stress on bubble surface

$u \quad$ velocity along $\mathrm{x}$-axis in Cartesian coordinates, $\theta$-axis in cylindrical coordinates

$U$ meniscus velocity

$U_{C L}$ contact line velocity

$U_{J} \quad$ capillary jump velocity

$v \quad$ velocity along $\mathrm{y}$-axis in Cartesian coordinates, $r$-axis in cylindrical coordinates

v velocity vector

$w \quad$ velocity along $\mathrm{z}$-axis

$\bar{w} \quad$ radially averaged velocity

$W \quad$ work required to bring molecules to a fluid-fluid interface

$W e \quad$ Weber number

$\nabla_{I I}$ del operator in transverse coordinates

$\delta \quad$ reduced Capillary number

$\theta \quad$ static contact angle

$\lambda \quad$ wavelength of light

$\lambda_{0} \quad$ wavelength of light in a vacuum

$\mu \quad$ dynamic fluid viscosity

$\rho \quad$ fluid density

$\tau \quad$ shear stress

$\sigma \quad$ surface tension between liquid and air

$\sigma_{S G}$ surface tension between solid and gas phase

$\sigma_{S L} \quad$ surface tension between solid and liquid

$\zeta \quad$ distance along beam axis 



\section{ABSTRACT}

THICKNESS MEASUREMENT OF DYNAMIC THIN LIQUID FILMS GENERATED BY PLUG-ANNULAR FLOW IN NON-WETTING MICROCHANNELS

David C. Deisenroth

Michigan Technological University, 2014

Advisor: Dr. Jeffrey S. Allen

Surface tension forces are significant at millimeter length-scales, causing profoundly different flow morphologies in microchannels than in macroscale flows. The existence and morphology of thin liquid films is particularly relevant for predicting performance and operational stability of devices containing microscale two phase flows. Analytical, computational, and experimental methods previously employed in the study of thin liquid films are discussed. Thicknesses before and after a novel film morphology, referred to as a 'shock,' are measured with a novel film thickness measurement technique that uses confocal microscopy. Film thicknesses predicted by previous work are compared to experimental results. Methods for increasing the accuracy of the confocal film thickness measurement technique are discussed. 



\section{INTRODUCTION}

\section{$1.1 \quad$ Introduction}

Microscale gas-liquid flow is utilized for many applications that require small channels with interior diameters less than $1 \mathrm{~mm}$. At this scale, surface tension forces are significant, which causes profoundly different flow morphologies in microchannels than in macroscale flows. The relative flow rates of gas and liquid in the channel determine the flow regime, which can transition from discrete bubbles to a annular film coating on the inside of the channel wall.

New applications of microscale two-phase flow are being developed, including small scale chemical reactors, biological research devices, and portable detection devices. The relative flow rates of gas and liquid affect the morphology of the liquid film generated in each regime. Particular flow regimes may produce the most desirable performance for each type of small-scale device. Discrete gas bubbles and liquid plugs are useful for small-scale chemical reactors [29]. A liquid film lining the channel wall with a gas core flowing through the center may be desirable for small scale heat exchangers [46]. Film thickness and morphology within PEM fuel cell passages also strongly affect performance [6]. Flow regimes may evolve through transient or start-up conditions within devices. Understanding film thickness and morphology in the large 
transition region between the annular and segmented regimes will aid in defining and characterizing performance limits of small scale devices, performance during startup conditions, and operational stability.

\subsubsection{Surface Tension}

Surface tension in at a gas-liquid interface arises because molecules at the surface lose half of their cohesive attractions [12]. This undesirable energy state is the reason molecules rearrange and form the smallest possible surface area. A molecule with cohesion energy $E$ is forced to a state of $E / 2$. If the molecule has an exposed area of $A_{M}^{2}$, the surface tension is of order $\sigma \cong E /\left(2 A_{M}^{2}\right)$ [12]. Surface tension is mechanically described by a quantity proportional to the number of molecules brought to the surface to increase surface area. The amount of work $(\delta W)$ required is proportional to the increase in surface area $(\delta A)$, as shown in equation $1.1[12]$.

$$
\delta W=\sigma \cdot \delta A
$$

This relation produces surface tension in SI units of $\mathrm{mJ} / \mathrm{m}^{2}$, which represents work per surface area. The relation is commonly reduced to force per unit length (tension), producing $\mathrm{mN} / \mathrm{m}$.

\subsubsection{Wettability}

Surface wettability is a product of a the molecular interaction of liquid with a solid substrate and the surrounding fluid phase. Wettability describes the tendency of a liquid to spread or repel from a surface. A perfectly wetting liquid tends to spread 
across an entire surface and a non-wetting liquid tends to form a discrete drop. Wettability changes with each combination of liquids, surfaces, and surrounding fluids. A spreading parameter, $S$, describes the wettability of a fluid with a solid, surrounded by another fluid (typically air). The spreading parameter of a drop of liquid on a flat solid surface surrounded by air is found with equation $1.2[12]$.

$$
S=\sigma_{S G}-\left(\sigma_{S L}+\sigma\right)
$$

where $\sigma_{S G}$ is the surface tension between the solid and gas phase, $\sigma_{S L}$ is the surface tension between the solid and liquid, and $\sigma$ is the surface tension between the liquid and air. A liquid is totally wetting when $S>0$ and partially wetting when $S<0$ $[12$.

\subsubsection{Contact Angle}

A sufficiently small drop of partially wetting or non-wetting liquid forms a drop in the shape of a spherical-cap on the surface. The angle at which the surface of the drop appears (macroscopically) to intersect the flat surface is defined as the static contact angle $(\theta)$. A drop with $\theta \leq \pi / 2$ said to be "partially wetting" and $\theta>\pi / 2$ is said to be "non-wetting." Contact angle is measured internal to the liquid relative to the substrate, as illustrated in figure 1.1.

Contact angle is related to the spreading parameter $(S)$ by the Law of YoungDupré, as shown in equation $1.3[12]$.

$$
S=\sigma(\cos \theta-1)
$$




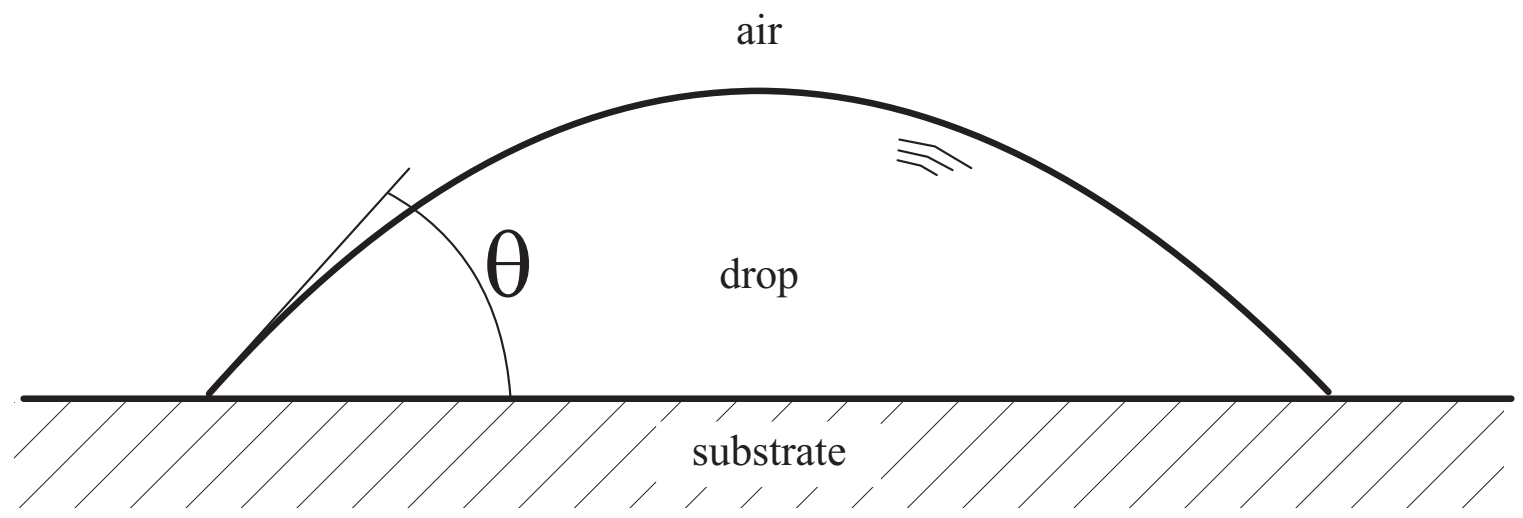

Figure 1.1. Illustration of apparent contact angle of a partially wetting liquid drop.

Contact angle also characterizes the angle of intersection of a meniscus inside a capillary. Contact angle changes when the intersection of the solid, liquid, and gas phase (contact line) is moving, as occurs in a translating drop, a growing/shrinking drop, or a moving meniscus. An advancing contact line is driven forward by the fluid behind it ("behind" relative to the direction of motion); a receding contact line is withdrawn by the fluid ahead of it. An advancing contact angle tends to be larger than $\theta$ and a receding contact angle tends to be smaller than $\theta$. Wettability can be reversed if a surface is pre-coated with the liquid, i.e. a wetting liquid can be changed to non-wetting and vise versa [12].

\subsubsection{Scaling Parameters}

With small length scales, the magnitude of surface tension forces can be significant compared to other forces. Several common scaling parameters are used to characterize the relative magnitude of fluid properties in two-phase flows.

The magnitude of gravitational forces relative to surface tension is characterized by the Bond number $(B o)$, as shown in equation 1.4 . 


$$
B o=\frac{\Delta \rho g l^{2}}{\sigma}
$$

where $\Delta \rho$ is density difference across the interface, $g$ is acceleration due to gravity, $l$ is a characteristic length scale (typically channel diameter or drop radius), and $\sigma$ is surface tension. A Bo much less than one indicates that surface tension determines interface shape, and is relatively unaffected by gravity. Low Bo regimes in channels are generally axisymmetric, even in horizontal orientations.

The capillary length $\left(l_{c}\right)$ is closely related to the $B o$, as shown in equation 1.5$][12]$.

$$
l_{c}=\sqrt{\frac{\sigma}{\Delta \rho g}}
$$

If the characteristic length scale is smaller than the capillary length $\left(l>l_{c}\right)$, surface tension is the dominant factor determining interface shape.

Capillary number $(\mathrm{Ca})$ shows the relative magnitude of viscous forces compared to surface tension, as shown in equation 1.6 .

$$
C a=\frac{U \mu}{\sigma}
$$

where $U$ is meniscus velocity, $\mu$ is liquid viscosity, and $\sigma$ is surface tension. Viscous forces become important and begin to distort a moving meniscus when $\mathrm{Ca}>10^{-3}$ 32 .

The relative magnitude of inertial forces compared to surface tension is characterized by Weber number $(W e)$, as shown in equation 1.7 .

$$
W e=\frac{\rho U^{2} l}{\sigma}
$$


where $\rho$ is liquid density, $U$ is meniscus velocity, $l$ is a characteristic length scale (typically meniscus/drop radius), and $\sigma$ is surface tension. A $W e$ much less than one indicates that inertial forces are insignificant.

\subsubsection{Two-Phase Flow Regimes}

Microscale two-phase flow regimes can be categorized by flow morphology, in which similar morphologies are separated into regimes. The primary flow regimes of interest here are plug, plug-annular, and annular. Plug flow consists of discrete plugs of liquid separated by gas bubbles. At sufficiently high velocity, the gas bubbles are encapsulated with a liquid film lining the channel wall. Annular flow consists of a gas core flowing through the center of the channel with an annular film coating the length of the channel. Therefore, plug-annular flow is a combination of plug and annular flow, consisting of a "wavy" annular film coating the channel wall with periodic liquid plugs. Waves in the annular film are sometimes referred to as "lobes." The plugannular regime is generated by growth of lobes until the gas core will "pinch-off" to form a liquid plug. This process may occur periodically, generating periodic plugs. The general appearances of the three flow regimes considered here are illustrated 1.2 .

Plug, slug, and annular will be the only regimes considered here, and the "slug" regime will henseforth be refered to as "plug-annular." Capillaries of $1.6 \mathrm{~mm}$ inside diameter were used in the experiments performed by [5]. These somewhat larger capillaries may produce less axisymmetric flow than capillaries with $500 \mu \mathrm{m}$ inside diameter. The transition between plug and annular flow may appear "slug-like," which is likely why the regime was labeled as such. In the experimental setup used by [5], $B o \cong 3.5$, indicating that gravitational forces are significant compared to surface 

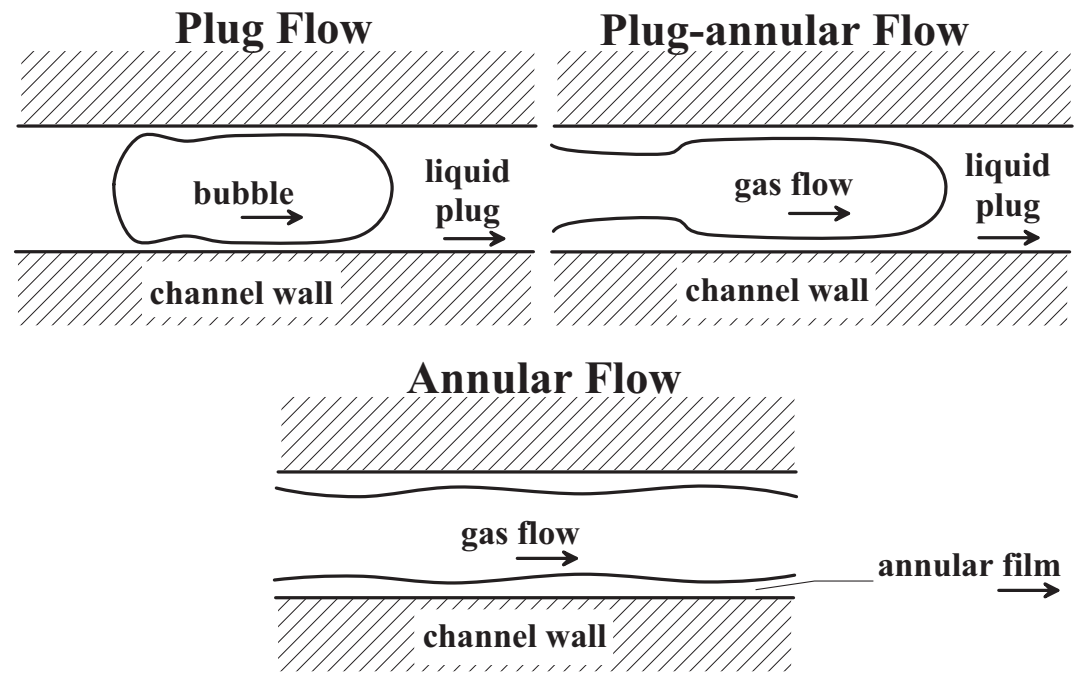

Figure 1.2. Illustrations of the plug, plug-annular (slug), and annular flow regimes.

tension, and may produce less axisymmetry in the unstable plug-annular regime than lower Bo flows.

In low $B o$ flows, morphology is most strongly affected by fluid viscosities and surface tension. In addition, contact angle has significant implications for flow morphology and flow regime transitions, particularly when the liquid film ruptures [26, 56]. Contact angle effects have also been studied indirectly for cases of contamination or textured surfaces [48, 57]. Figure 1.3 describes one categorization of these flow regimes for varying gas/liquid flow rates and contact angles [5].

In figure 1.3 , the contact angle $(\theta)$ is observed to affect the transitions between flow regimes. The plug-annular flow regime represents a large portion of the possible flow regimes for low Bond number two-phase flow. Therefore, a better understanding of the flow regime will aid in developing devices utilizing microscale two-phase flow. The thickness of liquid films existing within each regime may be used compare experimental data with mathematical models which predict film thickness and morphology. 


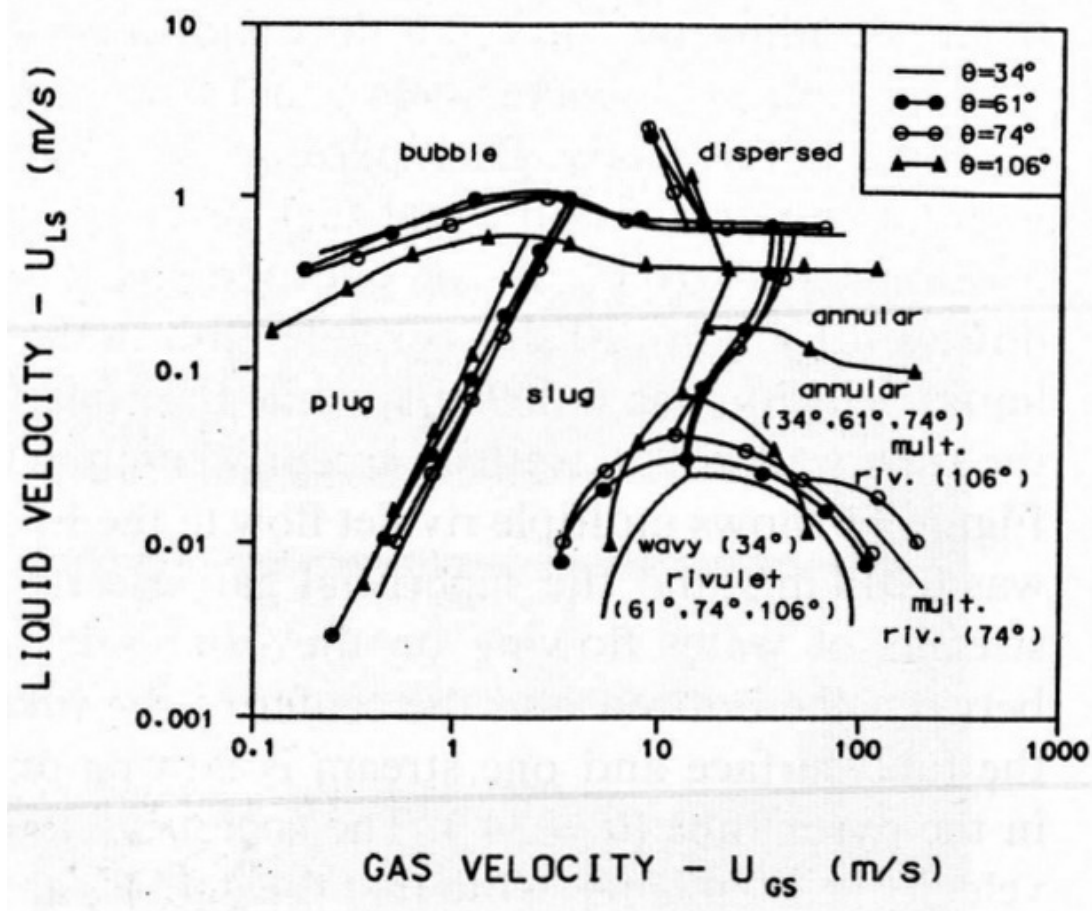

Figure 1.3. Two-phase flow regimes as a function of liquid velocity, gas velocity, and contact angle [5]. Reprinted by permission, copyright agreement attached in Appendix A. 


\subsection{Two-Phase Flow Research}

Film thicknesses generated by gas-liquid flows have been studied dating back to the early 1900's. Study methods include experiments, analytical modeling, and computational modeling. Film thickness generated by plate withdrawal from a liquid bath was an early topic of interest, and has been studied on various wettability substrates at a variety of withdrawal speeds. Gas-liquid flows in channels has also been studied at a variety of gas and liquid flow rates. Understanding the dynamics of plug-annular flow in non-wetting microchannels requires an introduction to the research topics involved, including plug flow, annular flow, contact line dynamics, and dewetting films.

\subsubsection{Liquid Film Deposition Behind a Receding Meniscus}

A receding liquid meniscus (meniscus is withdrawn relative to a substrate by liquid behind it) may deposit a liquid film or the contact line may move with the meniscus [31]. For the case of film deposition, the liquid is said to be "entrained." Entrainment and film morphology depends on $C a$ and the static contact angle.

\subsubsection{Wetting Film}

A wetting film entrained on a substrate does not require analysis of the contact line region, because in theory a contact line does not exist as $\theta=0$. Experiments have shown that a wetting drop will produce a very thin film that quickly spreads along a static substrate, and will increase the spreading rate of the bulk film [13], indicating $\theta=0$. The study of these very thin liquid films is generally done on 


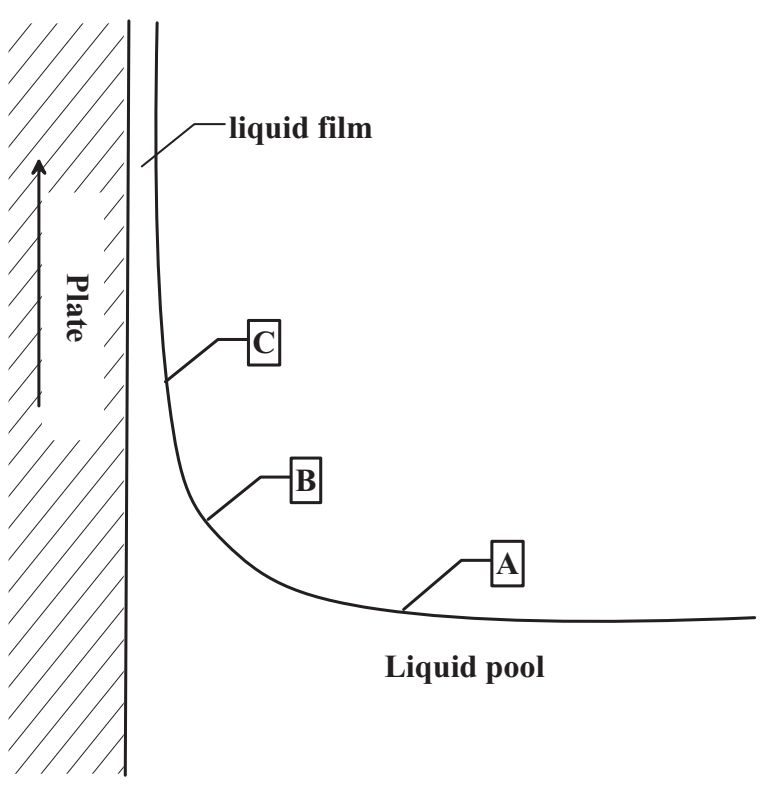

Figure 1.4. Illustration of the Landau-Levich problem.

the molecular level because continuum assumptions break down. Very thin liquid films (nm thickness) behave differently than thin liquid films ( $\mu m$ thickness) because intermolecular forces become important 61].

Landau and Levich were some of the first to suggest that film thickness behind a receding meniscus is a function of $\mathrm{Ca}$. The Landau-Levich [36] problem consists of a plate withdrawn vertically from a pool of liquid at low $C a$, as illustrated in figure 1.4 .

It is assumed that $\theta=0$ and gravity is negligible. As the plate is withdrawn, the static meniscus (A to B) smoothly transitions into the film via a region termed the "dynamic meniscus" (B to $\mathrm{C}$ ). The curvature of the dynamic meniscus slowly decreases moving from B to C. A curved surface under tension manifests as Laplace pressure, which is defined in equation $1.8[12$.

$$
\Delta p=\sigma\left(\frac{1}{R_{1}}+\frac{1}{R_{2}}\right)
$$


where $\Delta p$ is the change in pressure across the interface, $R_{1}$ and $R_{2}$ are the orthogonal curvatures of the interface. It is assumed that the curvature of the dynamic meniscus and the static meniscus are approximately equal. Equilibrium between Laplace pressure and viscous forces is represented by equation 1.9 [12].

$$
\frac{\mu v}{h^{2}} \cong \frac{\sigma}{d_{B C} l_{c}}
$$

where $\mu$ is viscosity, $v$ is the velocity of the plate, $h$ is the film thickness, $d_{B C}$ is the length of the dynamic meniscus, and $l_{c}$ is the capillary length. The length $d_{B C}$ is proportional to the other two length scales in the problem, as shown in equation 1.10 [12.

$$
d_{B C} \propto \sqrt{h l_{c}}
$$

Which simplifies to the final form of the Landau-Levich film thickness relation, as shown in equation 1.11 [12].

$$
h \cong 0.94 C a^{2 / 3}
$$

The assumptions in the derivation of this expression limit the applicability of this model to $\mathrm{Ca}<10^{-3}[12$.

\subsubsection{Non-wetting Film}

A drop deposited on a substrate with $\theta>0$ produces a visible and static contact line region. A contact line region also exists with a receding meniscus. Depending on the speed of recession, the contact line may move with the meniscus to generate 
a static meniscus shape or the contact line may move more slowly than the meniscus to generate a film region [31]. The contact line region a dynamic film adds a level of complexity to modeling the film thickness. Non-wetting films may develop complex morphology due to contact line dynamics.

[32] noted there is a stress and pressure singularity at a moving contact line when modeling with the no-slip boundary condition. This singularity is one of the challenges in modeling films with $\theta>0$. The mechanism which makes the singularity nonphysical is molecular reordering very near the contact line, requiring a parameter or boundary condition to accurately model this nanoscale phenomena on a macroscopic scale. [11] used a slip length based on a macroscopic length scale. The Navier slip condition is another way to remove contact line singularity, and is illustrated in equation 1.12 [54.

$$
\left.u\right|_{z=0}=l_{s} \frac{\partial u}{\partial z}
$$

where the $z=0$ is the location of the wall and $l_{s}$ is the slip length. The slip length for liquids has been derived from statistical molecular theory as equation 1.13 [54].

$$
l_{s} \sim \frac{\mu D a k_{B} T}{\left[\sigma a^{2}\left(1+\cos \theta_{e}\right)\right]^{2}}
$$

where $a$ is molecular size, $D$ is a self-diffusion coefficient, $k_{B} T$ is a measure of thermal energy, and $\theta$ is the static contact angle [7, 54]. The Navier slip condition and slip length provide parameter to eliminate the contact line singularity. This definition of the slip length is not universally agreed upon, and the definition of the slip length can significantly influence macroscale film morphology [37. [31] observed that an impossibly small slip parameter would have to be used for the author's model to 
agree with experimental results pertaining to the critical speed of film deposition, which is discussed below. The slip length was also shown to have a significant impact on the model prediction of the critical capillary number at which the contact line moves with the substrate indefinitely [9].

Molecular dynamics at the contact line profoundly affect film morphology. 53] used a mean field continuum description to show that contact angles are selected by intermolecular forces. Contact angle hysteresis (changes in contact angle due to drop volume changes) were also shown with this molecular treatment. [14] used asymptotic matching to find a solution incorporating the local contact line solution with the farfield meniscus for both an advancing and receding meniscus on a plate.

Dynamic contact angles represent another way of describing the shape of a contact line region without much consideration of molecular dynamics. [32] suggested the dynamic contact angle is influenced by a function based on the static contact angle. [32] also noted that contact angle decreases with a receding contact line, and increases with an advancing contact line.

At a critical capillary number the contact line will move along the substrate more slowly than the traveling meniscus, forming an entrained liquid film. [43] experimentally showed that receding contact line velocity may be a function of equilibrium contact angle for liquids/substrate combinations with $\theta>0$. [14] showed that a static solution vanishes above a critical capillary number, indicating the contact line is moving away from the static meniscus of a plate withdrawn from a liquid reservoir. As reported by [14], a commonly adopted model for describing the transition to entrainment is a reduced capillary number $(\delta)$ developed by [40], shown in equation 1.14 . 


$$
\delta=3 \frac{C a}{\theta^{3}}
$$

It is found that this wetting transition often under predicts the speed at which entrainment occurs, and may be attributed to contact angle hysteresis occurring on real surfaces [14].

[52] re-derived a relation for film thickness without the classical lubrication approximation used for small contact angles. Above approximately $\theta \geq 30^{\circ}$, the model predicts much thicker films than predicted with lubrication theory.

\subsubsection{Plug Flow}

The film deposited behind a receding meniscus in a capillary tube is very similar to that of a plate withdrawn from a liquid pool. As the meniscus recedes, a film is deposited on the substrate. At sufficiently high $\mathrm{Ca}$, the film connects two consecutive plugs forming a bubble encapsulated in liquid film, as illustrated in figure 1.5 .

In general, the bubble is characterized by several regions. Region A to B closely matches a meniscus which is unaffected by viscous forces, and is therefore termed the "static meniscus." Region B to C is again the transition from the static menisus to the

film, and again is termed the "dynamic meniscus." Region $\mathrm{C}$ to $\mathrm{D}$ is an approximately the flat film region. Between $\mathrm{D}$ and $\mathrm{E}$ is a characteristic undulation near the rear meniscus.

[8] developed an accurate and widely accepted analytical model of plug flow in microchannels. Many characteristics of the bubbles were determined as a function of $C a$, including bubble profile and internal pressure. Film thickness as a function of $C a$ will be the only parameter discussed here. 


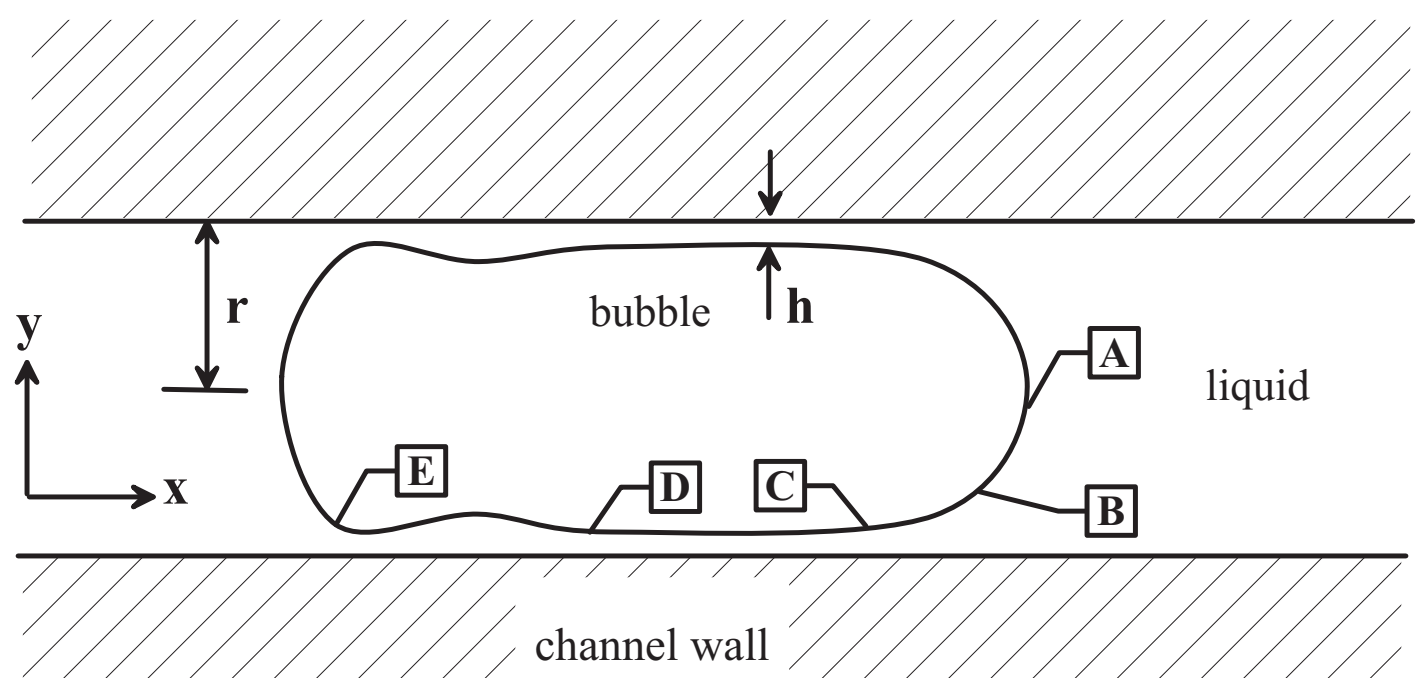

Figure 1.5. Illustration of a bubble moving through a capillary tube.

The first assumptions are that $\theta=0$, the ratio of film thickness to channel radius $(h / r)$ is small, gravity is negligible, and inertial effects are negligible. Next, it is assumed that $d x / d y<<1$ in the region $\mathrm{B}$ to $\mathrm{C}$, meaning that the dynamic meniscus is modeled as a flat film. For this reason, $u_{x}$ and $u_{x x}$ can be neglected. Under these conditions, the Navier-Stokes equation is [8]:

$$
u_{y y}=\frac{1}{\mu} \frac{d p}{d x}
$$

The pressure condition at the surface of the bubble is taken as equation 1.16 [ 8 .

$$
p=-\sigma \frac{d^{2} y}{d x^{2}}-\frac{\sigma}{r}
$$

The no-slip boundary condition means that $u=0$ when $y=0$. After several integrations and substitutions, equations 1.15 and 1.16 are simplified to the final form of the film thickness relation, equation 1.17 [8]. 


$$
\frac{h}{r_{c}}=1.34 C a^{2 / 3}
$$

Due to the assumptions made in the derivation of this relation, the applicability is limited to $C a<10^{-3}[8]$.

A notable extension of Bretherton's work uses an arclength-angle formulation [42]. In this derivation, azimuthal flow is neglected, as are gravity and inertia. The scaled Navier-Stokes equations are presented as:

$$
\begin{gathered}
C a \nabla_{I I}^{2} w=\frac{\partial p}{\partial z} \\
\nabla_{I I} p=0
\end{gathered}
$$

where $\nabla_{I I}$ is the del operator in transverse coordinates. No-slip boundary conditions of the liquid on the channel and pressure normal to the bubble surface are imposed. A detailed asymptotic expansion and eigenfunction analysis developed film thickness and bubble profiles for a range of $\mathrm{Ca}$. The solution, expressed in terms of eigenvalues, matches Bretherton's solution up to $C a=3 \times 10^{3}$ and is more accurate up to approximately $C a=1$ for low $W e$.

The effect of inertia was examined later. By eliminating the assumptions of no inertia and $h>>r$, [4] reworked the Bretherton problem and developed the expression shown in equation 1.20 .

$$
\frac{h}{r_{c}} \cong \frac{C a^{2 / 3}}{1+C a^{2 / 3}-W e}
$$

The model expresses the relative magnitude of inertial forces in film thickness.

Another approach to determining film thickness behind a receding meniscus uses 
the film surface evolution equation [45]. The full evolution equation is presented as equation 1.21 .

$$
\left(h-h_{\infty}\right)=\left(\frac{h^{3}-h_{\infty}^{3}}{3}\right)\left(\frac{\rho g}{\mu v}\right)-\frac{h_{m}^{2}}{h}\left(\frac{\sigma}{\mu v}\right) \frac{d h}{d x}-\frac{h^{3}(\sigma / \mu v)}{3} \frac{d^{2} \sin \theta}{d x^{2}}=0
$$

where $h_{\infty}$ is the value the film thickness when $y \rightarrow \infty$ and

$$
h_{m}^{2}=\frac{A_{L L}^{[6]} G_{6}(\theta)-A_{S L}^{[6]}}{6 \pi \sigma}
$$

$A_{L L}^{[6]}$ is the liquid/liquid Hamaker constant, $A_{S L}^{[6]}$ is the solid/liquid Hamaker constant for a truncated [6-12] Lennard-Jones potential, and $G_{6}$ is an angular correction function [45]. The full evolution equation is then nondimensionalized and augmented, neglecting gravity, forming equation 1.23 .

$$
\frac{d^{3} \hat{H}}{d \hat{X}^{3}}-\frac{3 A}{\hat{H}^{4}} \frac{d \hat{H}}{d \hat{X}}+C a\left(\frac{\hat{H}-\hat{H}_{\infty}}{\hat{H}^{3}}\right)=0
$$

where $\hat{H}=h / l_{c}, \hat{X}=x / l_{c}$, and $A=h_{m}^{2} / l_{c}^{2}$. In equation 1.23 the first term represents capillary components, the second term represents molecular components, and the third term represents the viscous component. The expression is numerically solved and film thickness is plotted as a function of $C a$ for $10^{-8}<C a<10^{-6}$. At low $C a$ (approximately $C a<10^{-6}$ ), the model predicts thicker a film thickness which becomes independent of $C a$. The constant value of film thickness at low $C a$ depends on the magnitude of the intermolecular force [45. This constant film thickness departs from the Landau-Levich model, which approaches 0 as $\mathrm{Ca} \rightarrow 0$.

The relative complexity of analytically modeling two-phase plug flows makes com- 
putational modeling appealing for research and characterization. An early effort to solve the "Bretherton" problem of plug flow used the Navier-Stokes equations, simplified as follows in equation 1.24 through 1.26 [50].

$$
\begin{gathered}
\frac{\partial u}{\partial z}+\frac{1}{r} \frac{\partial}{\partial r}(r v)=0 \\
\rho \frac{\partial u}{\partial t}=-\frac{\partial p}{\partial z}-\left(\frac{1}{r} \frac{\partial}{\partial r}\left(r \tau_{r z}\right)-\frac{\partial \tau_{z z}}{\partial z}\right) \\
\rho \frac{\partial v}{\partial t}=-\frac{\partial p}{\partial r}-\left(\frac{1}{r} \frac{\partial}{\partial r}\left(r \tau_{r r}\right)-\frac{\tau_{\infty}}{r}+\frac{\partial \tau_{r z}}{\partial z}\right)
\end{gathered}
$$

where $\tau_{z z}=-2 \mu \partial u / \partial z, \tau_{r r}=-2 \mu \partial v / \partial r, \tau_{\infty}=-2 \mu v / r$, and $\tau_{z r}=\tau_{r z}=-\mu(\partial u / \partial r+$ $\partial v / \partial z)$. Shear stress is denoted by $\tau$, and subscripts indicate direction. The velocity components $u$ and $v$ are in the $z$ and $r$ directions, respectively. The free surface (bubble surface) components are $\mathbf{T}=\mathbf{T}_{n} \mathbf{n}+\mathbf{T}_{t} \mathbf{t}$ and the boundary conditions are as follows [50]:

Streamlines at bubble surface:

$$
u \frac{\partial r}{\partial z}=v
$$

No tangential stress on the bubble surface:

$$
T_{t}=0
$$

Normal stress on the bubble surface due to pressure:

$$
T_{n} C a=\frac{1}{r\left[1+\left(\frac{\partial r}{\partial z}\right)^{2}\right]^{1 / 2}}-\frac{\frac{\partial^{2} r}{\partial z^{2}}}{\left[1+\left(\frac{\partial r}{\partial z}\right)^{2}\right]^{1 / 2}}
$$

The equations are then solved with quadrilateral nodes and isoparametric map- 
ping. Velocity field plots are generated for the nose of the bubble, and more importantly, the film thickness is reported as a function of capillary number for $5 \times 10^{-3}<$ $C a<1 \times 10^{-1}$. The film thickness calculations matches experimental data well for the entire $C a$ range. Inertial, but non-turbulent, flow fields partially explain the accuracy of the model in this $C a$ range.

Further studies of plug flow by computational methods were carried out later. A generalized and nondimensionalized Navier-Stokes equation is presented as follows in equation 1.31 through 1.32 [16].

$$
\begin{gathered}
R e \mathbf{v} \cdot \nabla \mathbf{v}=\nabla \cdot \mathbf{T} \\
\mathbf{T}=-\frac{p}{C a} \mathbf{I}+\left[\nabla \mathbf{v}+(\nabla \mathbf{v})^{T}\right] \\
\nabla \cdot \mathbf{v}=0
\end{gathered}
$$

The boundary conditions are set as no slip at the channel wall, symmetry about the channel centerline, and parabolic velocity profile far upstream from the bubble. The boundary conditions for the bubble surface include pressure normal to the surface and pressure due to surface tension curvature as follows [16]:

$$
\begin{gathered}
\mathbf{v} \cdot \mathbf{n}=0 \\
\mathbf{T} \cdot \mathbf{n}=\frac{1}{C a}\left(\frac{1}{R_{1}}+\frac{1}{R_{2}}\right) \mathbf{n}
\end{gathered}
$$

The equations are solved with Galerkin/finite element discretization [16].

Steamlines and bubble profiles are solved and film thickness is reported as a function of both Re and $C a$ for $5 \times 10^{-5}<C a<10$. The model is said to be comparable 
to Bretherton's for low $C a$ and good agreement with other work for higher $C a$. One of the biggest contributions of this work is development of a model which is accurate for a large $C a$ range and detailed discussion of flow properties ahead of the leading bubble meniscus. Using the same numerical technique, the authors also extended this work to the trailing bubble meniscus [17]. A more detailed simulation of streamlines and detached recirculations was done by $[23]$.

It is reported by [4] that [60] and 8] were the first to experimentally and quantitatively study plug flow in low Bond number (1mm diameter channels) systems. These two pioneering experimentalists set the standard for experimentation with, and measurement of, microscale plug flow. It can be assumed that all experiments are carried out with nonvolatile liquids or evaporation mitigation techniques are used, all experiments are in controlled temperature, glass channels are clean and dry for each test run, and the working liquid is uncontaminated. In these early experiments, two phase flow was photographed, and typically the photos were physically measured to convert the images into numerical data. In similar experiments, [10] encapsulated the working glass capillary with the same liquid as the working fluid to reduce distortion caused by refractive index step changes.

\subsubsection{Plug-Annular}

[2] observed the transition from annular flow to plug flow with water and oil. Rayleigh instabilities develop in the oil film under certain conditions. A critical wavelength is developed which predicts the growth of lobes in the film. At this critical wavelength, the lobes will grow until they pinch off and generate an oil plug. Periodic lobe formation along the length of the film was observed experimentally, and was shown 
to be in good agreement with the theory developed. It was also observed that lobes did not form in close proximity to the receding meniscus.

[25] studied the effect of surface wettability on annular film morphology with a liquid plug driven out of a channel. It was observed that a thicker film was deposited in channels with higher contact angles. Higher contact angles $\left(\theta=105^{\circ}\right)$ also produced less stable films which quickly rupture due to Rayleigh instabilities. A theory was developed, suggesting the film thickness in non-wetting channels is predicted by

$$
\frac{h}{r_{c}} \cong \frac{C a^{2 / 3}}{1-\theta^{2} / 2}
$$

which appear to hold true for moderate contact angles $\left(\theta<36^{\circ}\right)$ in the range of $10^{-4}<C a<10^{-} 2$. For high contact angles, it was shown that film thicknesses depart from the film thickness predicted by [8].

\subsubsection{Annular}

An early model of annular flow of a viscous, incompressible fluid flowing through another viscous, incompressible fluid (of different density and viscosity) was presented by [30]. The simplified Navier-Stokes equation for the core flow is presented in equation 1.36 and 1.37 [30].

$$
\begin{gathered}
\frac{\partial p}{\partial r}=0 \\
\frac{\partial p}{\partial z}-\rho g=\mu\left(\frac{\partial^{2} w}{\partial r^{2}}+\frac{1}{r} \frac{\partial w}{\partial r}\right)
\end{gathered}
$$

where $z$ is the axial direction and $w$ is the core fluid velocity in the axial direction. The differential system governing stability is presented as [30]: 


$$
\begin{gathered}
u_{t}+u u_{r}+\frac{v}{r} u_{\theta}+w u_{z}-\frac{v^{2}}{r}=-\frac{1}{\rho} p_{r}+\frac{\mu}{\rho}\left(\nabla^{2} u-\frac{u}{r^{2}}-\frac{2}{r^{2}} v_{\theta}\right) \\
v_{t}+u v_{r}+\frac{v}{r} v_{\theta}+w v_{z}-\frac{u v}{r}=-\frac{1}{\rho r} p_{\theta}+\frac{\mu}{\rho}\left(\nabla^{2} v-\frac{2}{r^{2}} u_{\theta}-\frac{v}{r^{2}}\right) \\
w_{t}+u w_{r}+\frac{v}{r} w_{\theta}+w w_{z}=-\frac{1}{\rho} p_{z}+g+\frac{\mu}{\rho}\left(\nabla^{2} w\right) \\
\frac{1}{r}(r u)_{r}=\frac{1}{r} v_{\theta}+w_{z}=0
\end{gathered}
$$

where $(u, v, w)$ are velocities in the $(r, \theta, z)$ directions, respectively, and subscripts indicate partial differentiation. Appropriate boundary conditions, including no-slip at the channel wall, are applied and this set of equations is solved with perturbation methods with asymmetric and axisymmetric perturbations. It is found that the system is stable with axisymmetric perturbations, but unstable with asymmetric perturbations, indicating that lobe formations will grow exponentially with asymmetric pressure/velocity disturbances in the system. The approach used in this analysis is linear, meaning that it only holds for very small lobe formations and is not applicable to severe lobe geometry.

More recently, models have also been developed for application to severe lobe topology, including film rupture. In a notable example, the film surface evolution equation was used to model an annular film, resulting in a generalized equation similar to that in equation 1.23 , which was numerically solved [33. In this model, the capillary is weakly curved, which results in non-axisymmetric flows. This curvature along the axis of the tube also resembles the effects of gravity because of curvature induced Laplace pressure. Axial perturbations were used to promote lobe growth and study the maximum wavelengths which promote lobe growth. This model allows for 
film rupture and drop formation, requiring the boundary conditions of the problem to be redefined. With redefined boundary conditions, growth and drainage of a single drop with $\theta=0$ was studied.

[34] modeled lobe formation fairly extensively, first solving the governing equations with linear perturbation theory, then by discretizing and solving the equations with finite element methods. The equations of motion are presented as follows [34]:

$$
\begin{gathered}
\frac{\partial r_{f}^{2}}{\partial t}=\frac{\partial}{\partial z}\left\{\bar{w}\left(r_{c}^{2}-r_{f}^{2}\right)\right\} \\
\frac{r_{c}^{2}-r_{f}^{2}}{2} \frac{\partial \bar{w}}{\partial t}+\frac{3 \mu r_{c} \bar{w}}{\rho\left(r_{c}-r_{f}\right)}=-\frac{r_{c}^{2}-r_{f}^{2}}{2} \frac{\partial}{\partial z}\left(\frac{\bar{w}^{2}}{2}+\frac{p}{\rho}\right)
\end{gathered}
$$

where $r_{f}$ is the film interior radius, $r_{c}$ is the tube interior radius, $\bar{w}$ is the radially averaged velocity, so that $w(r, z, t)=\bar{w}(z, t) f(r, z, t)$. Pressure due to surface tension curvature is:

$$
p(z, t)=\sigma\left\{-\frac{\gamma}{r_{f}}+\frac{\partial}{\partial z}\left(\gamma \frac{\partial r_{f}}{\partial z}\right)\right\}, \gamma=\left\{1+\left(\frac{\partial r_{f}}{\partial z}\right)^{2}\right\}^{-1 / 2}
$$

The solutions focus primarily on timescales of lobe growth and collapse, which are presented as a function of Re. The time to "liquid bridge," or closure of the capillary by convergent lobes is also determined. The time of lobe conversion marks the end of the applicability of the model due to severe morphology changes by surface reordering. The model was developed for researching lungs. Interestingly, this model is limited by the assumption that lobe perimeter does not change due to pulmonary surfactant. The surfactant changes surface tension and therefore contact angle of the working liquid on the substrate, requiring a morphology change and a nonzero contact angle to be integrated into the model. 
The stability of a thin annular film with gas a core at low $R e$ was experimentally observed by [3]. The test section consisted of a $54 \mu \mathrm{m}$ diameter glass tube and the working fluid was glycerol, a highly viscous fluid. The test section was encapsulated in glycerol to minimize optical distortions caused by refractive index step changes at interfaces. The lobe growth rate was reported as a function of dimensionless wave number. It was also observed that as lobes continue to grow they will merge at the center of the channel and form a plug. Secondary lobe formations were also observed, which are not predicted by mathematical models.

\subsection{Summary}

Film deposition on a perfectly wetting flat substrate is one of the earliest studies of film thickness. The plug flow regime has been studied extensively analytically, computationally, and experimentally. Annular flows have also been studied analytically, computationally, and experimentally. However, the plug-annular flow regime has been studied primarily experimentally, likely due to the difficulty in modeling film surface reordering in this regime. The effects of contact angle on film thickness and morphology are a topic of continuing study. 


\section{SCOPE AND GOAL}

Static contact angle has a significant affect on film thickness and morphology in microchannels. [24] observed a novel film morphology in microchannel plug-annular flow. The phenomena is referred to as a 'shock,' because a thin film jumps to a thicker film in a very short distance, as illustrated in figure 2.1. The objective of this research project is to measure the thickness of the thick and thin liquid films with confocal microscopy.

The film observed by 24 includes a receding contact line (A), a capillary ridge (A to $\mathrm{B}$ ), a thick film region (B to $\mathrm{C}$ ), and a thin film region (D to E). The shock between (C) and (D) appears to be a new phenomena which occurs in non-wetting channels. The evolution of the film morphology is shown in figure 2.2 .

The evolution of the plug-annular flow region is a repeating process. The receding contact line of a liquid plug moves more slowly than the meniscus causing a film region to develop. A capillary ridge occurs near the receding contact line. Within the film, the shock appears to move at a speed slower than the meniscus, but faster than the ridge. As the film region grows in length, the ridge grows in height and width to eventually pinch off and form a plug. The process then repeats. The physical

phenomena governing the film morphology is a complex combination of contact line dynamics, surface tension, viscosity, and inertia. 


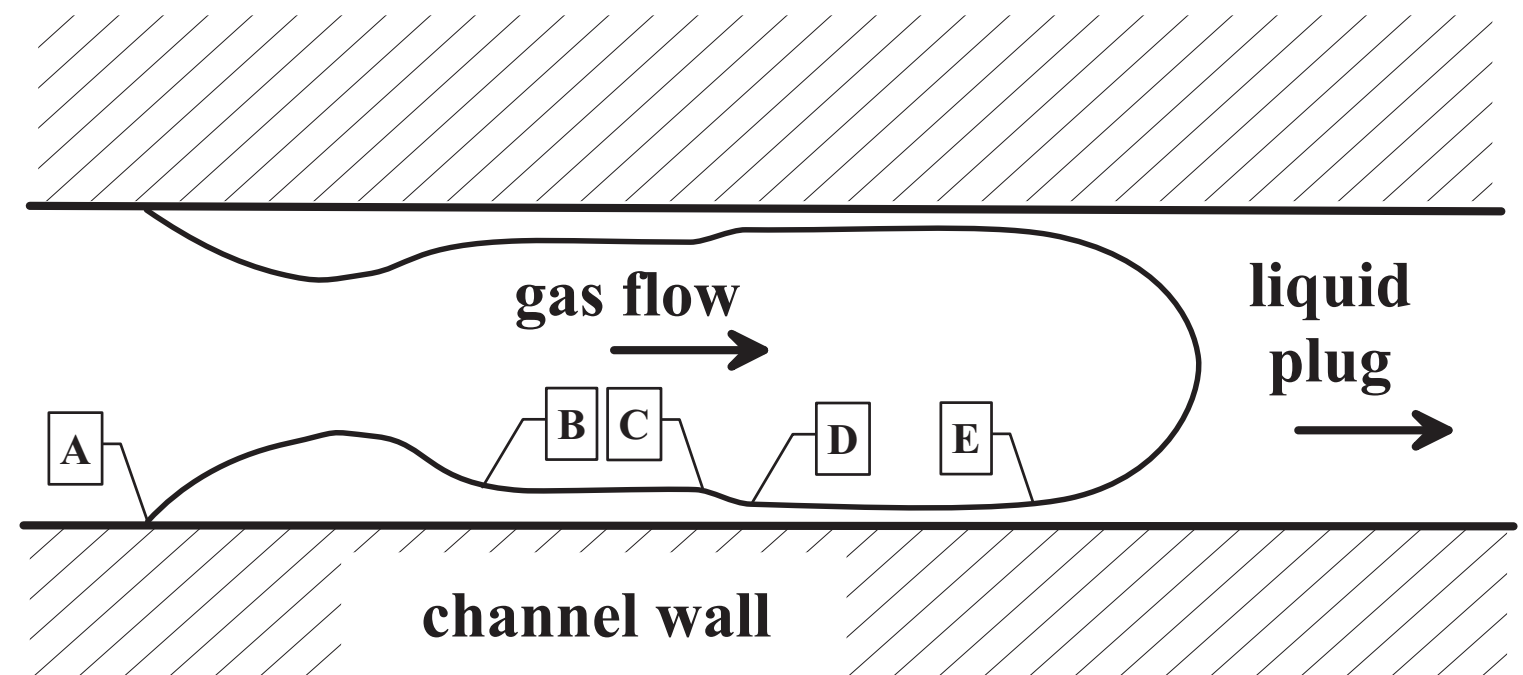

Figure 2.1. Plug-annular flow in a non-wetting microchannel. The receding contact line $(\mathrm{A})$ has $\theta>0$. The region between $(\mathrm{A})$ and $(\mathrm{B})$ is a capillary ridge, characteristic of a receding film on a partially-wetting or non-wetting substrate. The region between $(\mathrm{B})$ and $(\mathrm{C})$ is a thick film, thicker than the classical Bretherton film. Between (C) and (D) exists the shock, with a thin (Bretherton) film region between (D) and (E). 


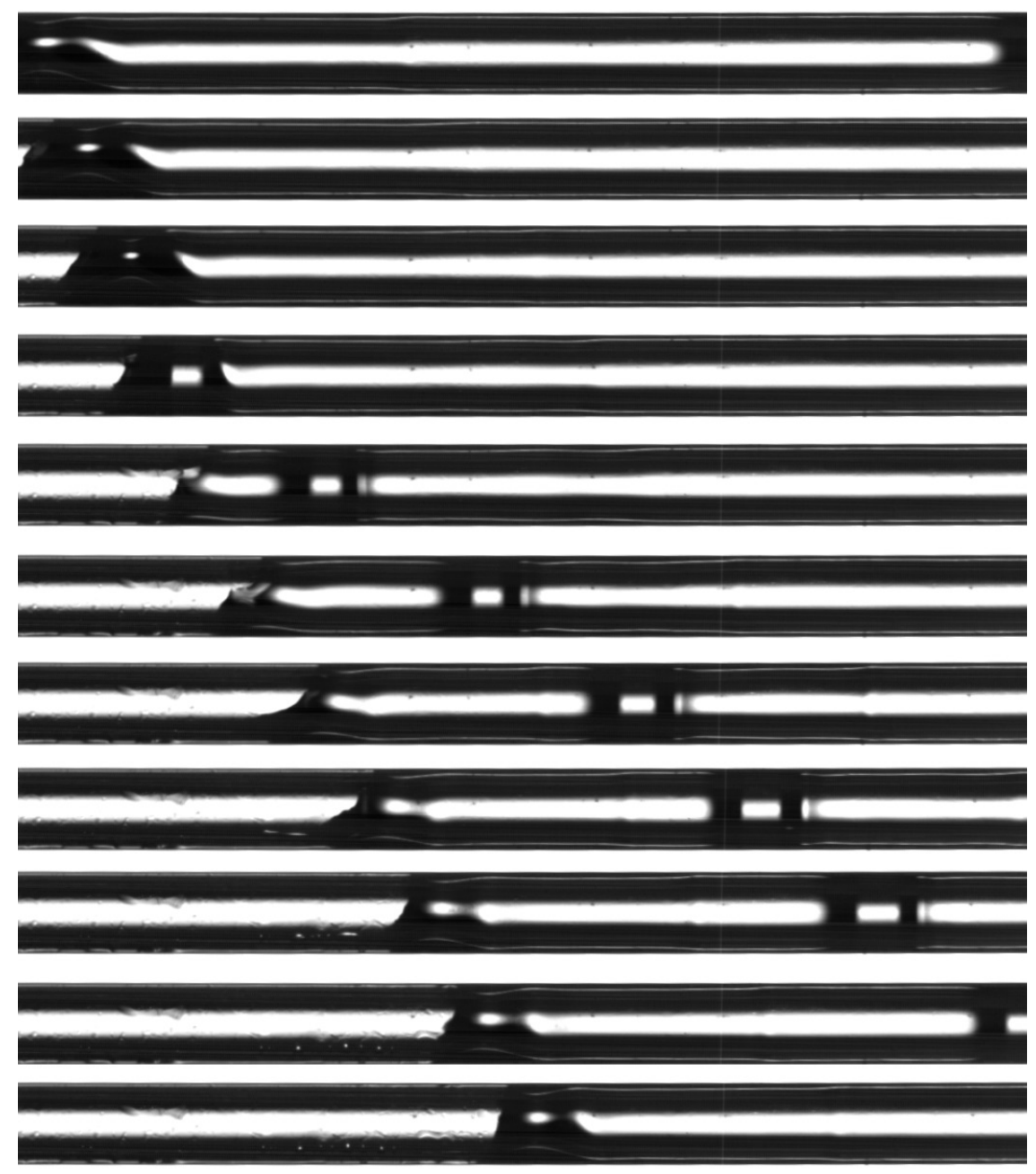

Figure 2.2. Shock structure formation in plug-annular regime with $C a=0.0172$. Image sequence is with $2 \mathrm{~ms}$ between frames and the channel length shown is $20 \mathrm{~mm}$ 
The capillary ridge phenomena (region A to B) has been observed with receding contact lines on non-wetting substrates. A hole nucleated in a non-wetting film will grow with time, which naturally produces a receding contact line. The contact angle will decreased from the static value to a constant value and the contact line will recede at a constant speed [43]. The capillary ridge is produced in the vicinity of the contact line region. The ridge is taller than the film height and the width of the ridge increases as the hole grows [43]. The shape of the ridge may be calculated with lubrication theory, as was done by [55]. A parabolic fit of the ridge constrained by the apparent contact angle was asymptotically matched to a Levich-Landau film.

[56] found a shock structure formation leading to a short flat film region near a receding contact line on a partially wetting plate withdrawn from a liquid bath. The working fluid was silicon oil and the substrate was a silicon wafer, generating $\theta=51.5^{\circ}$. Figure 2.3 illustrates the phenomena.

The film is characterized with several well-defined velocities: the substrate velocity, the contact line velocity, and the capillary jump (shock) velocity. The contact line velocity maintains a constant value relative to the moving plate, which is related to the critical speed of entrainment. Lubrication theory and dimensional analysis generate the relation shown in equation 2.1 for the thick film region [56].

$$
\frac{\mu}{\sigma} U_{j}=C a-\frac{h_{r}^{2}+h_{r} h_{L L}+h_{L L}^{2}}{3 l_{c}^{2}}
$$

where $U_{j}$ is the capillary jump velocity, $h_{r}$ is the flat film height, $h_{L L}$ is the LandauLevich film thickness, and $l_{c}$ is the capillary length. [56] suggests the thickness of the thick film region (the experimentally determined parameter $h_{r}$ ) may be a function of the equilibrium contact angle. It should be noted that this film morphology is not 


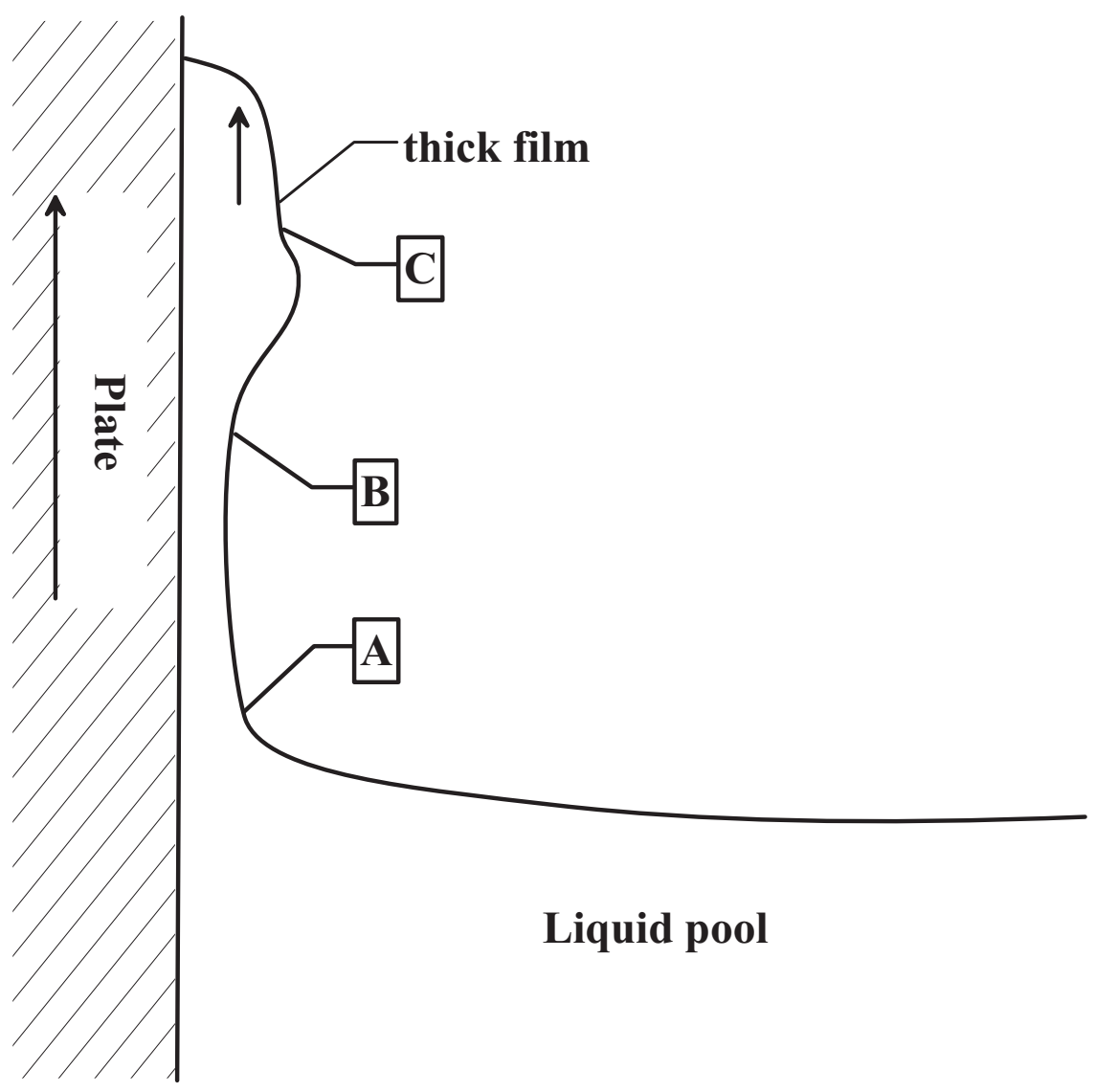

Figure 2.3. Non-wetting film morphology. [56] characterizes this film morphology as a Landau-Levich film between (A) and (B), a capillary jump between (B) and $(\mathrm{C})$, and a thick film region near the receding contact line 


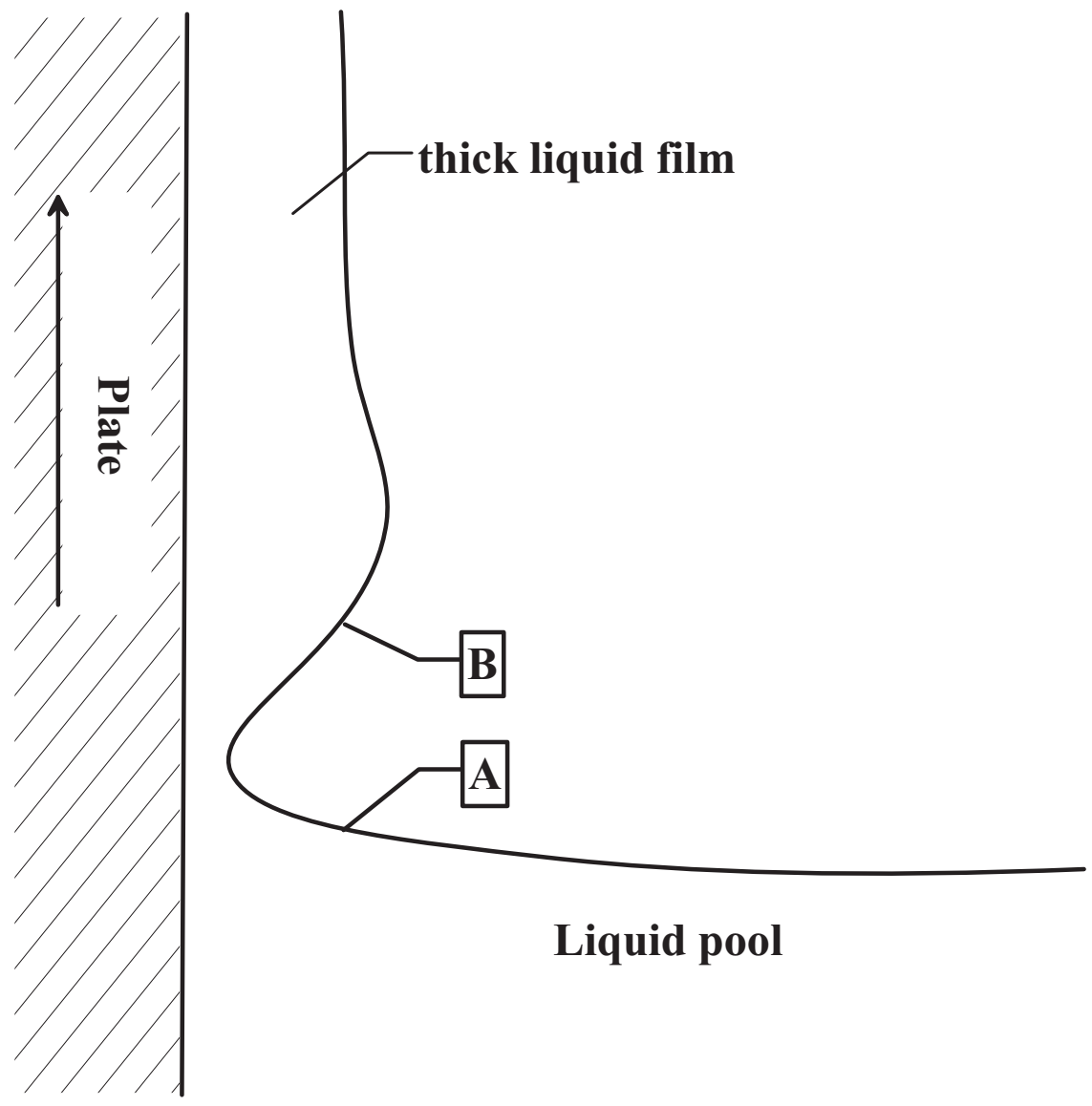

Figure 2.4. Non-wetting film morphology. [51] characterizes this film morphology describing the region between (A) and (B) as a dimple, with a thick film region above.

low Bond number and is significantly influenced by gravitational drainage.

The study of this thick viscous film region was later extended with the same liquid and substrate, but at a capillary number just above the critical value of contact line speed [51]. The effort was focused on the steady state region of the film, i.e. excluding the contact line region. The characteristic morphology of the experiment and model is illustrated in figure 2.4 .

The dimple region between (A) and (B) evolves to a Landau-Levich film at higher capillary numbers [51, 56]. The lubrication equation for free surface flows is used to 
develop equation 2.2 for the thick film region [56].

$$
h_{f} \cong(3 C a)^{1 / 2}-\frac{1}{2} Q C a^{2 / 3}
$$

where $h_{f}$ is the thickness of the thick liquid film, and $Q=q C a^{-5 / 3}$, and $q$ is the liquid flux along the plate. Good agreement between the model and experiment indicate that $h_{f} \sim C a^{1 / 2}$ for the thick film region entrained by a flat plate vertically withdrawn from a bath of partially-wetting liquid. Similar film morphologies involving a sharp capillary jump on a flat substrate have been modeled for the case of countercurrent gas-liquid flows [47] and for thermocapillary driven flows [58]. In both cases, the flows have a gravitational component.

One should note that substrate curvature in the direction perpendicular to liquid motion may have significant implications on film morphology, particularly for partially wetting or non-wetting substrates [9]. [9] used asymptotic matching to determine the maximum speeds of contact line motion for partially-wetting fibers of several orders of magnitude in diameter. The results show azimuthal curvature of the substrate may influence the critical speed of the contact line and the film morphology.

The primary interest of this research is to use the film thickness measurement technique developed by [28] to measure the film thicknesses generated in the plugannular flow regime in non-wetting microchannels and to compare the experimental results to the shock theory developed by [24]. [24] found that the shock observed in non-wetting channels $\left(\theta=105^{\circ}\right)$ does not occur in partially wetting channels $\left(\theta=30^{\circ}\right)$

[24] also observed that the hydraulic jump also only occurs above a critical capillary number, $C a=0.00357$. In addition, the the difference in the contact line speed 
and the receding meniscus speed must exceed a critical value. The criteria is

$$
\frac{U-U_{C L}}{c^{*}} \gtrsim 1
$$

where $U$ is the meniscus velocity, $U_{C L}$ is the contact line velocity, and $c^{*}$ is defined by

$$
c^{*}=\frac{1}{r_{c}-h} \sqrt{\frac{\sigma h}{\rho}}
$$

where $h$ is the thickness of the thick film region [24]. This model was developed based on wave front velocity and conservation of mass. The thickness of the thick film region is predicted to be

$$
\frac{h_{\text {nonwetting }}}{r_{c}}=7\left(\frac{h}{r_{c}}\right)^{\frac{4}{3}}
$$

where $\frac{h}{r}$ is the dimensionless Bretherton film thickness, which is also the thickness of the thin film region before the shock. The error in film thickness measured by [24 is estimated to be $20 \%$, which caused difficulty in testing the theory. The film thickness measurement technique developed by [28] may decrease the error in film thickness measurement to about 5\%, giving a more accurate analysis of the shock theory developed by [24].

The novel film thickness measurement technique uses confocal microscopy. By analyzing the reflection intensity of a film interface as a function of the objective lens location, film thickness can be precisely determined. The scanning speed of the Reflectance Swepth-Field Confocal (RSFC) microscope limits the speed at which film interfaces may be imaged, and therefore the number of images of a moving interface. 
Currently, the RSFC is limited to 200 frames per second, which allows for one image of the thick and thin film interfaces to be analyzed for each test run. An increased scanning speed would allow for multiple images of the thick and thin film interfaces to be averaged, and would therefore increase accuracy of the reflection intensity value. The information for increasing the scanning speed (potentially up to 3000 frames per second) of the RSFC provided by provided by Prairie Technologies, Inc. is included in Appendix B.

Increasing the imaging speed further may be accomplished by eliminating the physical motion of the scanning galvometer and therefore not scanning at all. With this method only a line the width of the laser would be imaged, which may be acceptable if only reflection-intensity data is required, and the speed could potentially be as fast as a high speed camera would allow. The repeatability of each test is also limiting because it introduces error in the Capillary number (the range of Capillary number observed) for which the test is valid. 



\section{Liquid Film Thickness MeAsurement}

\subsection{Current Research}

Two-phase flow systems represent a complex analytical problem, which is likely the reason little progress has been made in analytically modeling two-phase flows. Commonly used analytical methods generally require the redefinition of a problem to model flow regime changes. Finite element methods are useful for modeling complex morphologies and flow regime transitions. The current state of research in finite element two-phase flow modeling is in use of adaptive mesh to accommodate flow regime transitions [44].

Experimentation is used to characterize two-phase flow, particularly the transitions between stable flow regimes. Optical techniques are the most common method of measurement and characterization of microscale two-phase flows. A difficulty coupled with optical techniques is that light is refracted at refractive index step changes, particularly at liquid surfaces and menisci. Currently, techniques are in development to mitigate the interfacial lens effect to accurately measure properties of microscale flows and film thicknesses [38].

Front lighting, or epi-illumination, can be used to illuminated the interface from the same point it is viewed, reducing the lens effect which occurs when light is trans- 
mitted though the medium. Epi-illumination can be used with white light, florescence microscopy, and confocal microscopy. Confocal microscopy filters out-of-focus light, producing high-quality images without noise from out-of-focus image planes. Confocal microscopy has been adapted for film thickness measurement [28]. Magnetic resonance imaging has also been adapted for microscale liquid film thickness measurement [38].

Interferometry is a method which uses the constructive and destructive interference of light to measure distances between refractive index step changes. This method can also be used to characterize topology of surfaces for annular flows [39].

Commercially available Laser Focus Displacement meters (LFD) have been used to measure film thicknesses in micro-scale flows under a variety of conditions [59]. This method measures the intensity of reflected light to locate refractive index step changes, and therefore, interface locations. This method may also be used to develop surface topologies at lower flow speeds. To accurately measure film thickness with a LFD, a geometric optics correction is required. In annular flows, wave height and wave frequency have been reported as a function of Reynolds number [59]. The LFD technique has also been adapted to document bubble profiles at various Capillary numbers [19, 22]. The LFD technique has further been adapted to document film profiles of plug flow in square microchannels [21]. Finally, the effect of bubble acceleration on liquid film thickness profile has been explored with the LFD technique [20].

The power spectra of a laser reflection from a liquid film in annular flow has also been used to determine wave frequencies as a function of Re [1]. This work also detected secondary, short lived, wavy structures in the annular film, which have not been predicted computationally [1]. 


\subsection{Introduction to Confocal Microscopy}

Confocal microscopy is commonly used in biological research applications for its ability to filter light from out-of-focus image planes, effectively creating and "optical slice." Light from the sample is transmitted through a barrier filter and into an imaging device. Barrier filters may be pinhole-shaped for spinning disk confocal units or slit-shaped for swept-field confocal units. Microscopes come in several illumination configurations, primarily transmittance and reflectance. In transmittance illumination, light is transmitted from an external light source through the sample and into the objective lens. In reflectance illumination, or epi-illumination, light is transmitted from the objective lens, reflected from the sample, then back into the objective lens. This experiment utilizes a Reflectance Swept-Field Confocal (RSFC) microscope. The RSFC microscope uses swept-field illumination, meaning that a thin line of laser light is swept across the camera chip as it records. Confocal scanning and camera imaging are synchronized. Illumination light is $488 \mathrm{~nm}$ in wavelength. Figure 3.1 illustrates how confocal out-of-focus light is filtered out with confocal microscopy.

The current experiment uses reflectance swept-field confocal microscopy to measure the reflection intensity from a refractive index step change as a function of objective lens location. The optical slice location changes with the location of the objective lens, which changes the intensity of light imaged by the camera attached to the RSFC. The highest reflection intensity locates the RI step change, and therefore the channel wall and film interface. It should also be noted that light is also refracted at each change in refractive index along the beam path; refraction is not shown in figure 3.1 for simplicity. A detailed discussion of accounting for refraction is detailed in section 3.5 . 


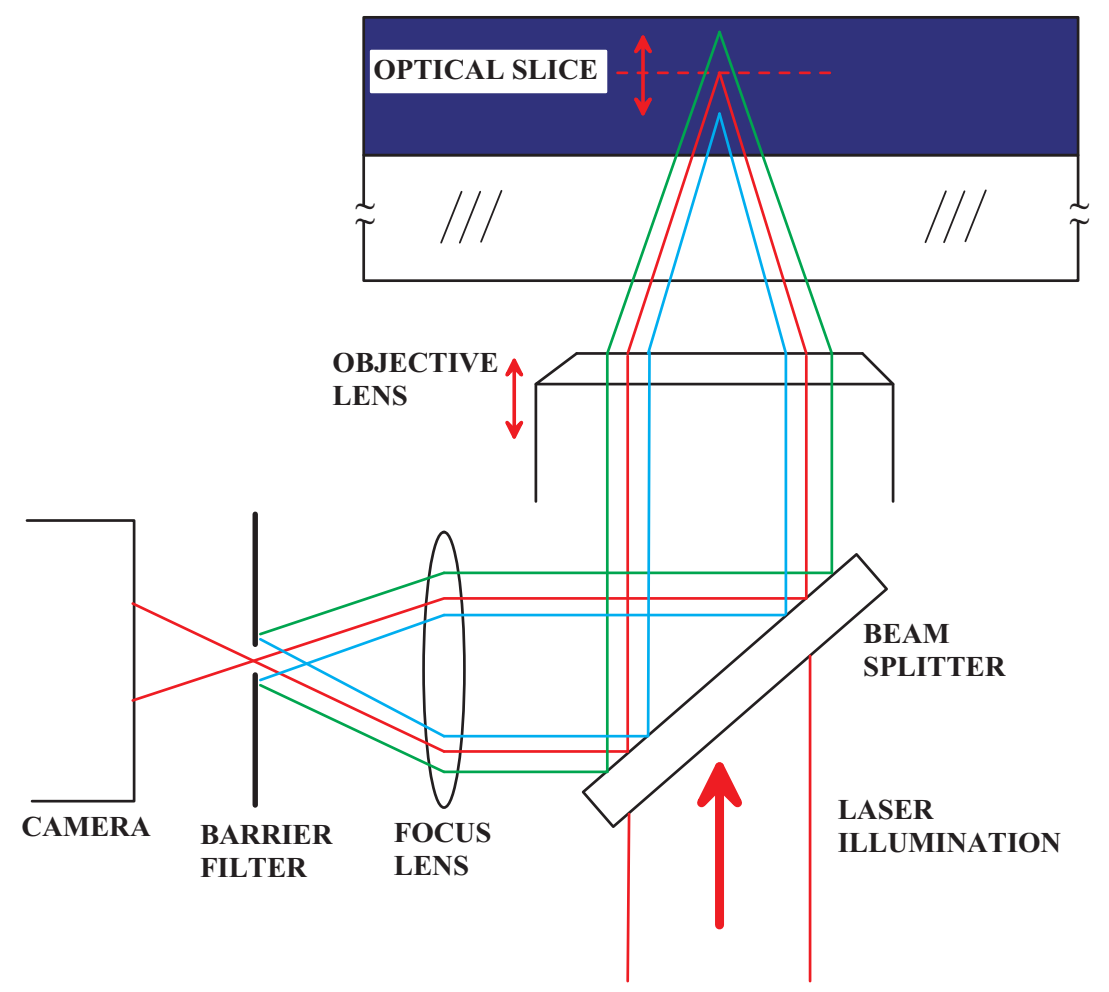

Figure 3.1. Confocal microscopy uses a barrier filters out-of-focus light generating an optical slice 


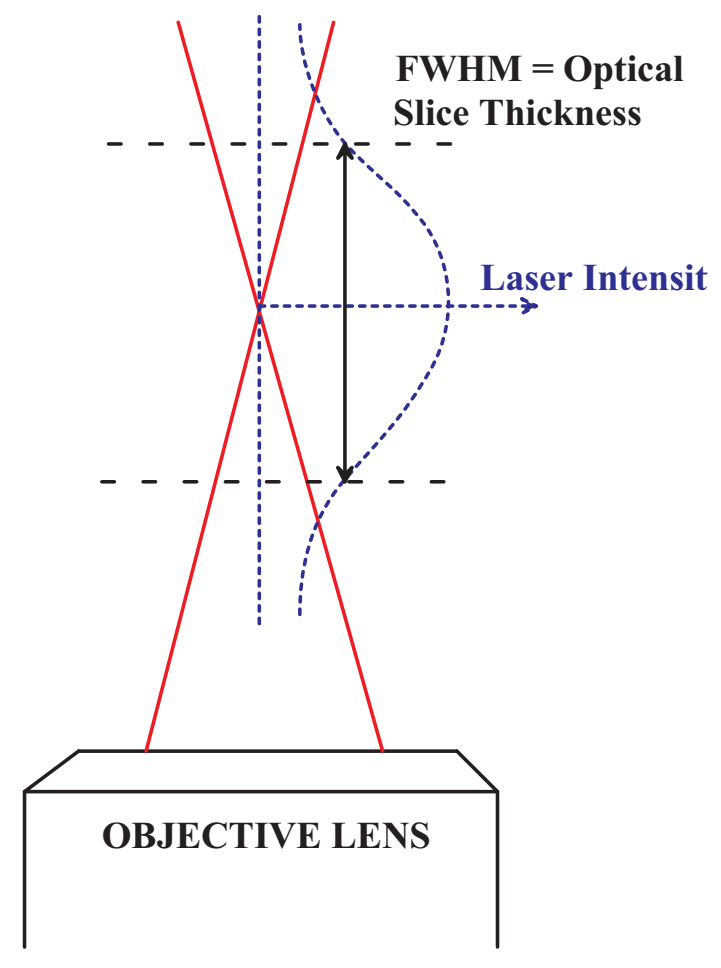

Figure 3.2. Optical slice thickness is determined by the full width of a Lorentzian curve at one-half the maximum intensity

\subsubsection{Optical Slice}

The optical slice is a volume of light which is thin in the axial direction and fills the camera view field. This volume of light is does not have ridged boundaries, but instead the light intensity fades along the laser axis in a Lorentzian distribution [49]. Figure 3.2 illustrates an approximation of the laser beam path, along with a qualitative description of the laser intensity along the beam path.

By convention, the thickness of the optical slice is taken as the full width of a Lorentzian distribution of the intensity profile at one-half the maximum intensity, referred to as full-width, half maximum (FWHM) [18]. For many application of confocal microscopy, optical slice thickness (FWHM) is a useful parameter used for experiment 
design and analysis. It should be noted that light from outside the FWHM optical slice thickness may be detected with fading intensity. The optical slice thickness is determined by the objective lens, confocal barrier filter, and excitation wavelength. The optical slice thickness has been tabulated by [27] and is $9.84 \mu \mathrm{m}$ with the $20 \times$ objective lens with a $35 \mu \mathrm{m}$ slit barrier filter, and excitation wavelength of $488 \mathrm{~nm}$. The $10 \times$ objective generates an optical slice thickness of approximately $86.6 \mu \mathrm{m}$ with the same settings.

The thickness of the optical slice relative to film thickness has implications for the reflection intensity detected by an imaging device. At each refractive index step change, light is both reflected and transmitted into the medium. The power transmitted and reflected at a refractive index step change normal to a beam path may be be approximated by

$$
\begin{gathered}
P_{t}=\frac{2 n_{1}}{n_{1}+n_{2}} \\
P_{r}=\frac{n_{1}-n_{2}}{n_{1}+n_{2}}
\end{gathered}
$$

where $P_{t}$ and $P_{r}$ are the fractions of the original power which are transmitted and reflected, respectively. The refractive index of the transmission medium is $n_{1}$ and the incident medium is $n_{2}$. Reflection of light at each refractive index step change causes a decrease in laser intensity transmitted into each new medium. This fact becomes particularly relevant when the optical slice incorporates two refractive index step changes, such as the channel wall-water interface and the water-air interface of the liquid film. Light reflecting from the channel wall-water interface increases the total reflection intensity observed by the imaging device, which is particularly noticeable 
for thin films and relatively thick optical slices. For cases in which the optical slice thickness is low compared to the film thickness, light reflected from the channel wall may not be detected.

\subsubsection{Cross View-Field Intensity}

The optical slice may be a non-planar shape because focused light travels with a spherical wavefront. For this reason, the optical slice detected herein may represent a curved volume. The effect of wave-front curvature is minimized by cropping images to an area which represents the highest $5 \%$ of reflection intensity with the optical slice centered on the interface. This way, a small nearly planar area of the spherical wavefront is analyzed.

The cross view-field intensity profile is closely related to the optical slice shape. To better understand this intensity profile, a first-surface mirror was imaged at multiple optical slice locations. An image with the optical slice centered on the surface of the mirror is shown in figure 3.3 .

The intersection of the areas of interest in the $\mathrm{x}$ and $\mathrm{y}$ direction represent the area of interest for measuring film thicknesses, i.e. all images of film interfaces are cropped to this region. It may be observed that the intensity profiles in the $\mathrm{x}$ and $\mathrm{y}$ directions are not perfectly flat. Intensity profiles in the region of interest in the confocal view field are plotted in figure 3.4 and figure 3.5 .

It may be observed that the intensity profiles at different optical slice locations (is below the mirror surface, + is above the mirror surface) maintain similar profiles, but with differing intensity. The peak intensity is observed at $0 \mu \mathrm{m}$, indicating the optical slice is centered on the reflective surface. The changes in intensity across the 


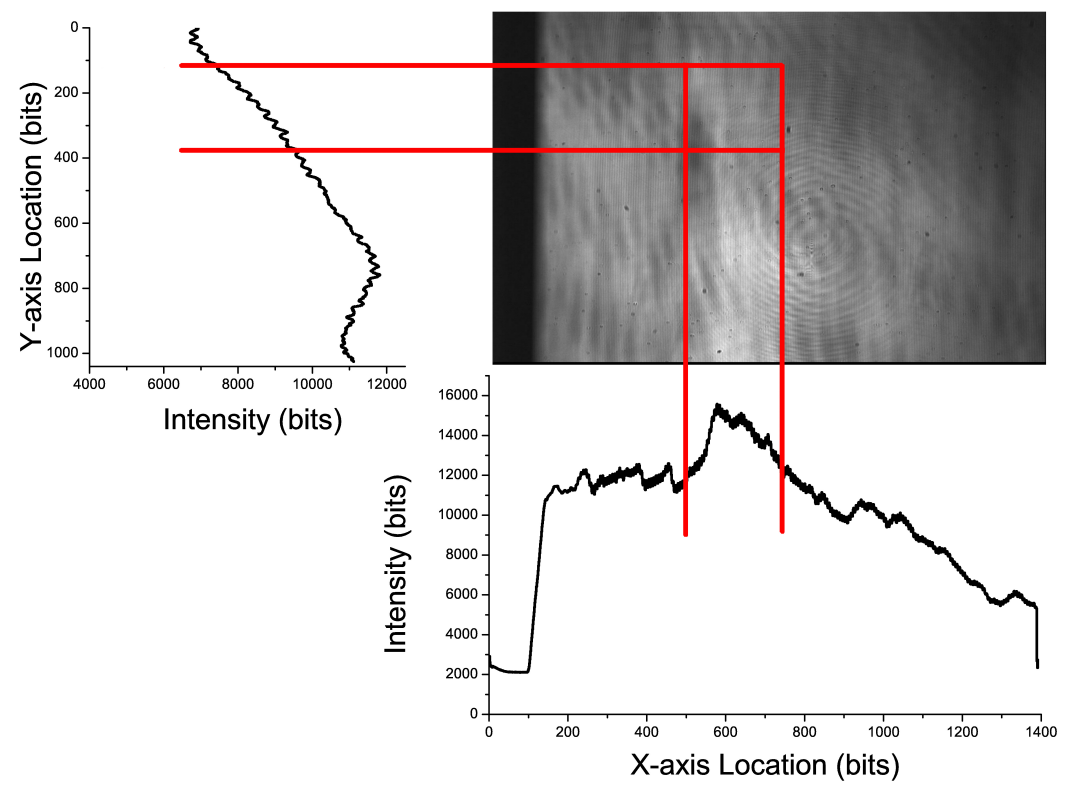

Figure 3.3. Cross view-field intensity of the confocal imaging system

confocal view field may be attributed to the alignment of internal optical components, and possibly the shape of the optical slice.

\subsubsection{Thickness Measurement of a Known Medium}

To demonstrate the viability of the technique, the thickness of a known medium was measured. The medium used was a borosilicate cover slip, $150 \mu \mathrm{m}$ in thickness. Images were gathered in $0.25 \mu \mathrm{m}$ increments ranging from below the bottom surface to above the top surface. The intensity profile as a function of objective lens $(10 \times)$ location is shown in figure 3.6 .

It may be observed that the peak reflection intensity from the bottom of the cover slip is higher than the peak intensity from the top surface. This change in intensity is partially due to the fact that the light reflected from the bottom surface is not 


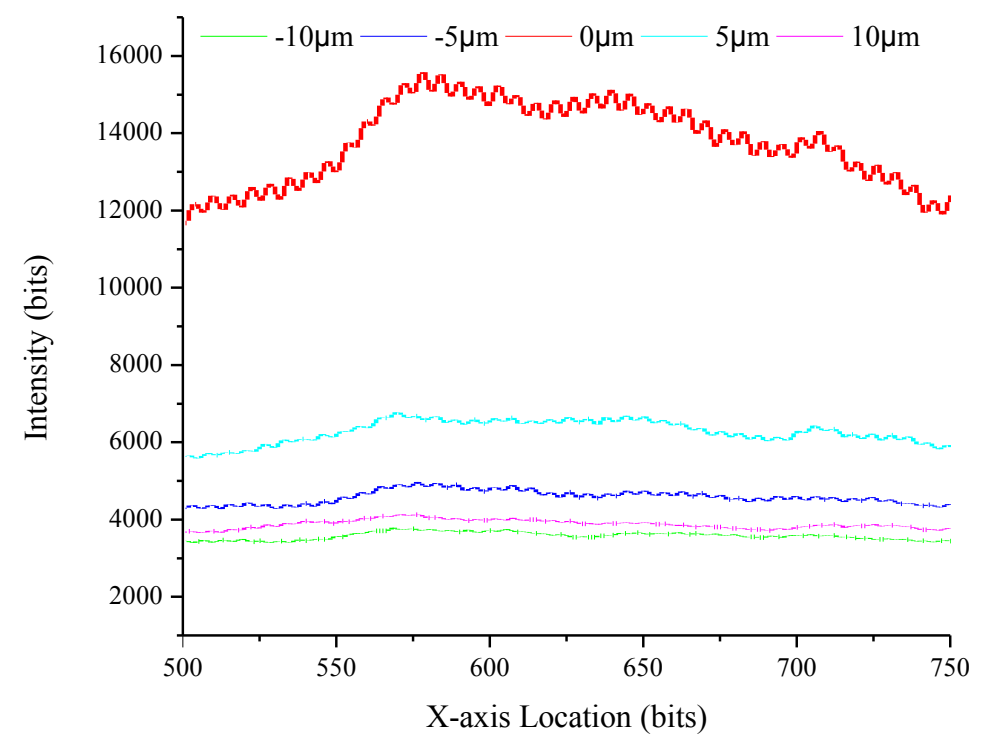

Figure 3.4. Cross view-field intensity along the x-axis in the region of interest. The optical slice location of $0 \mu \mathrm{m}$ indicates the optical slice is centered on the mirror, thus the brightest intensity profile. 


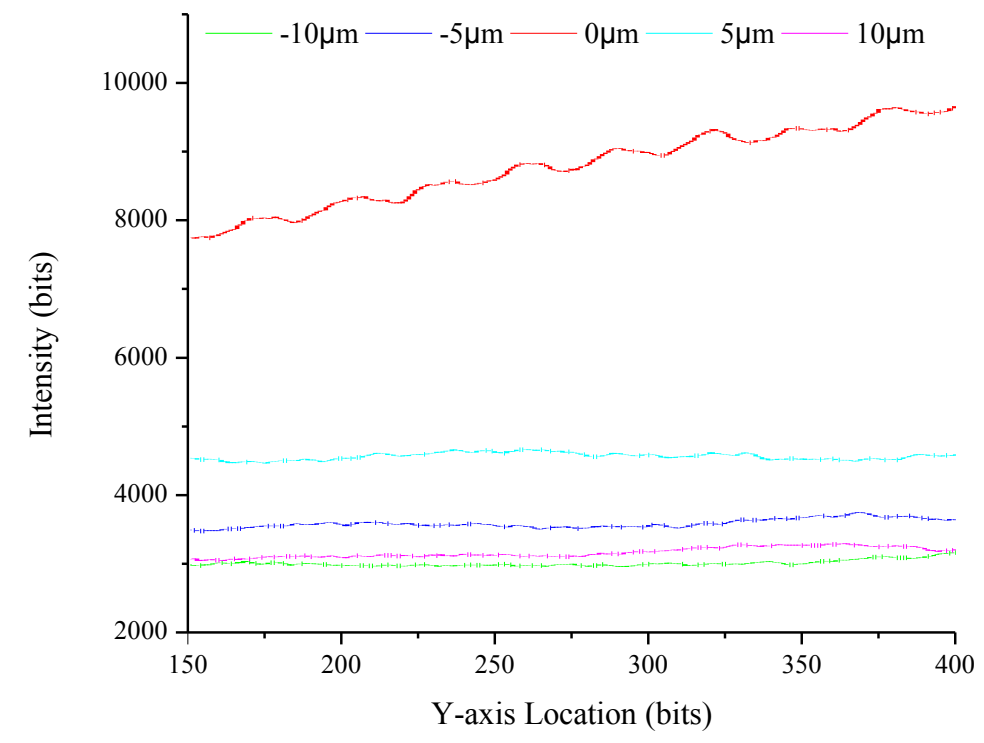

Figure 3.5. Cross view-field intensity along the y-axis in the region of interest. The optical slice location of $0 \mu \mathrm{m}$ indicates the optical slice is centered on the mirror, thus the brightest intensity profile. 


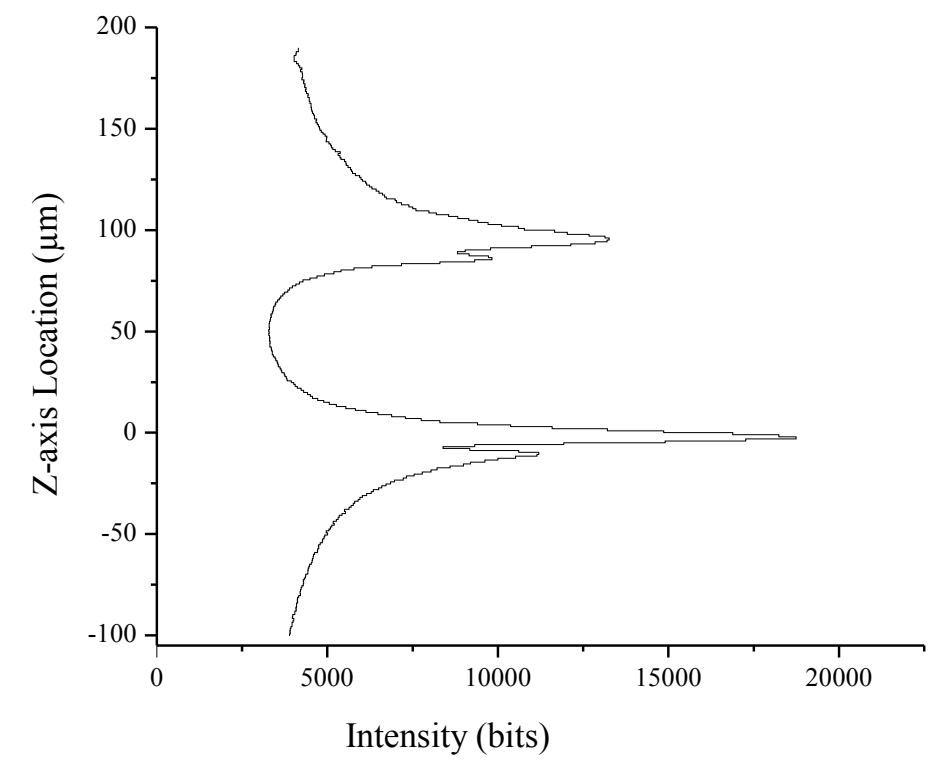

Figure 3.6. Intensity profile imaged by stepping the $10 \times$ objective lens upward through a $150 \mu \mathrm{m}$ thick borosilicate cover slip.

transmitted to the top surface, generating a weaker reflection from the top surface.

The peak-to-peak intensity represents the cover slip thickness as a function of objective lens position. The objective lens position is then correlated with the optical slice change in position with geometric optics. Using the small-angle approximation, the geometric optics correlation factor is 1.474, indicating a cover slip thickness of $154.3 \mu \mathrm{m}$. The error between the cover slip thickness and the measured value are within the uncertainty in the thickness of the cover slip. The accurate thickness measurement of a known thickness sample indicates that interfaces may be located by reflection intensity with confocal microscopy. 


\subsection{Experimental Setup}

The experimental setup consists of a cylindrical test section which contains the two-phase flow of interest. The test section tubing is encapsulated with Sigma-Aldrich glycerol, which is refractive index matched to borosilicate glass with $\mathrm{RI}=1.474$. Refractive index matched liquid minimizes refraction and fringe patterns generated by curved refractive index step changes in the radial direction.

A Nikon TE-2000U inverted microscope outfitted with a Prairie Technologies swept-field confocal unit for this experiment; this setup will be referred to as the RSFC microscope. The RSFC microscope transmits light into the test section where it is reflected from RI step changes, also known as Fresnel reflection. Interfacial reflections were imaged with a high speed camera and flow morphology was imaged with a macro-view camera located above the test section. The intensity of Fresnel reflections are then used to locate the interior channel wall and gas-liquid film interface, effectively measuring film thickness. Figure 3.7 illustrates the experimental setup.

Standard wide-field imaging is used to view the test section from above to capture a macroscopic view of the flow morphology. The flow is assumed to be axi-symmetric, justified by $B o=0.34$, although in the plug-annular regime sometimes can vary from axi-symmetric. Water is driven out of the test section by pressurized ultra high-purity nitrogen. The outlet of the test section runs into a hydrostatic head of approximately 3 inches, which acts as a stabilizing mechanism. A laser filter (laser-gog lens) is used to shield the macro-view camera from the laser light which is transmitted through the test section. 


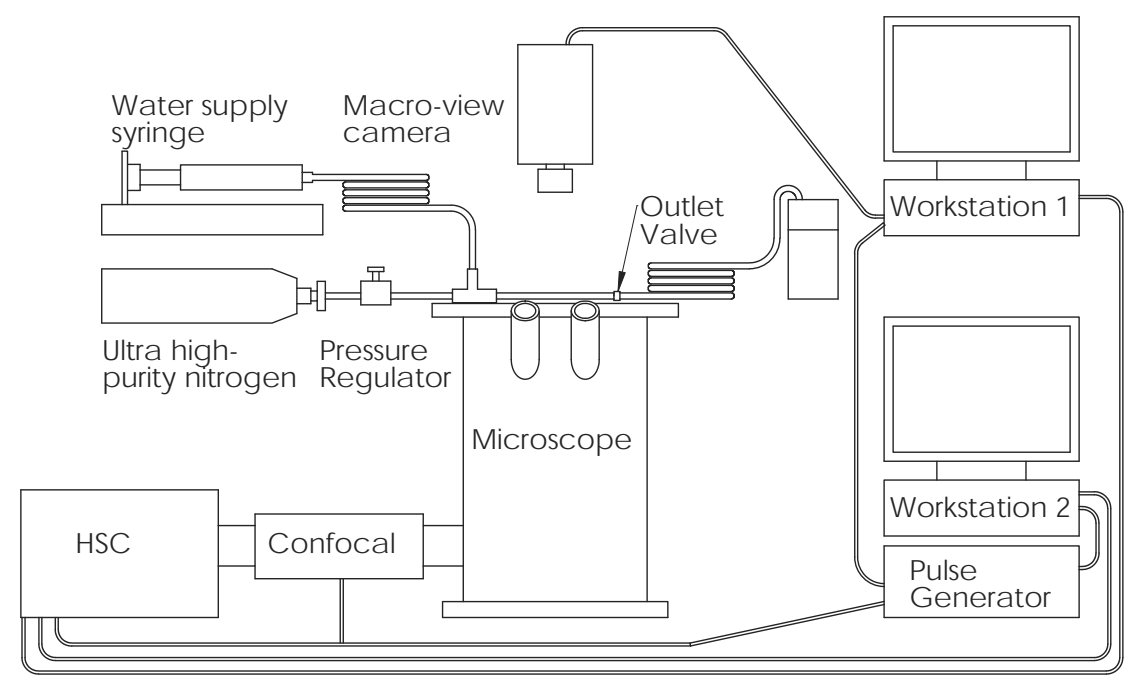

Figure 3.7. An overview of the experimental setup: interfacial reflections are imaged with the confocal microscope, which is synchronized with the attached high-speed camera, and controlled through workstation 1. Macro-view images are gathered with a camera located above the test section. The macro-view camera computer triggers the confocal high-speed camera image capture and macro-view image capture.

\subsubsection{Test Section}

A Vitrocom CV5070-100 borosilicate glass tube was used as a test section. The tubing is $0.5 \mathrm{~mm}$ inside diameter, $0.7 \mathrm{~mm}$ outside diameter, and $10 \mathrm{~cm}$ long. The inside of the tubing was coated with Rain-X®, a polydimethylsiloxane based hydrophobic glass coating. To coat the interior of the capillary, a method developed by [24], was used. The capillary was dipped in a drop of the coating and wicked into the capillary to its capillary length, approximately $2 \mathrm{~cm}$. The capillary was then overturned and the plug traveled to the other end due to gravity. The coating was then dried by passing ultra high-purity nitrogen through the test section at approximately 10psig for 3 minutes. This process was repeated 3 times. Finally, the test section was rinsed with $60 \mathrm{~mL}$ of distilled water. This coating generates $\theta=105 \pm 5^{\circ}[24$. 


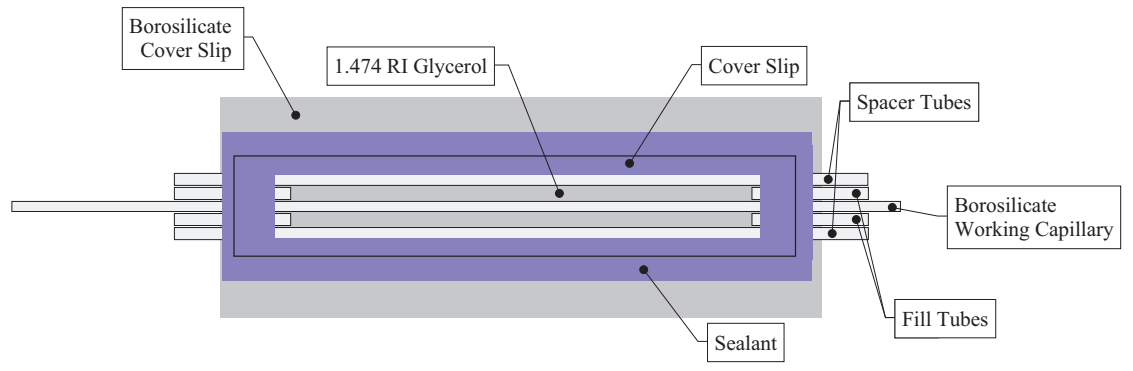

Figure 3.8. Test section constructed to reduce refraction of the beam path from the bottom of the cover slide to the channel wall

The non-wetting test section was then encapsulated with glycerol to create a continuous refractive index beam path from the bottom of the borosilicate cover slide to the channel wall-liquid interface. The construction of the test section is illustrated in figure 3.8 .

The encapsulated test section reduces spherical aberration and constructive interference due to the exterior channel wall, increasing the accuracy geometric optics relations (detailed below) and reducing noise with in the image data.

Plug-annular flow was generated by filling the working capillary with distilled water, then driving it out with pressure-regulated ultra high-purity nitrogen gas. The nitrogen flow pressure was regulated with a Bellofram Type 70 0-2psig pressure regulator. A Wikai 0-3psig pressure gauge was used in conjunction with the pressure regulator. Pressures of $0.60 \mathrm{psig}, 0.75 \mathrm{psig}$, 0.80psig driving the liquid plug $7.5 \mathrm{~cm}$ from the entrance of the test section generated the flow regime of interest at, $C a=0.00419$ $C a=0.00702$, and $C a=0.01015$, respectively. 


\subsubsection{Imaging}

\subsubsection{Macro-View}

A Jai Pulnix TM1325CL 10-bit camera was located directly above the test section, equipped with Fujinon TV 1:1.4/16 lens for tests with $C a=0.00702$ for a macroview. Spacers were used between the lens and camera to reduce the depth of focus to approximately $10 \mathrm{~cm}$. The macro-view camera was used to determine flow morphology and the capillary number of the leading meniscus of the plug-annular region. At 30 frames per second, the macro-view camera captures approximately 4 images of the meniscus as it travels through the $4 \mathrm{~cm}$ view field. A laser filter was located between the macro-view camera and the test section to avoid direct exposure of the camera imaging chip. The test section was illuminated from above with a Cuda model I-150 light source, which provided enough light for a $1 / 4000$ s shutter speed.

A Photron high speed camera with a Edmund Industrial Optics $2.5 \times$ long-working distance microscope was used for the macro-view camera for tests with $C a=0.00419$ and $C a=0.01015$. The frame rate was 400 frames per second, and the light intensity provided by the external light source allowed for a shutter speed of $1 / 2000$ s. The view field diameter was $7 \mathrm{~mm}$.

\subsubsection{Confocal View}

Confocal images were gathered with a Photron APX RS high-speed camera (HSC). A $2 \times$ magnification lens was located between the confocal output and the high-speed camera to better fill the large HSC imaging chip. Confocal scanning and image capture were synchronized by triggering each device with a $200 \mathrm{~Hz}$ signal with a 0.80 
duty cycle from a National Instruments BNC-2110 data acquisition device combined with a custom LabView program. The start of image capture for the macro-view camera and HSC was triggered by setting the strobe settings of the macro-view camera to output upon trigger. This output was delivered via a trigger card in the macroview camera computer to the general input of the HSC, which was then set as the triggering mechanism.

\subsubsection{Experimental Procedure}

The first experimental step is finding the z-axis location (referring to the objective lens location) of the interior channel wall with confocal imaging. With an empty (dry) test section, the approximate channel wall location can be found with inspection. The objective lens is then moved down $10 \mu \mathrm{m}$. One image is gathered, then the objective lens is moved upward $1 \mu \mathrm{m}$ and the process is repeated. The channel wall is imaged at twenty z-axis locations. The images are then processed (detailed in section 3.4) to precisely locate the channel wall.

The experimental procedure used here is similar to the method the author codeveloped with [28]. The channel is initially filled with water and pressurized ultra high-purity nitrogen drives the liquid out of the tube. The flow is imaged $7.5 \mathrm{~cm}$ from the inlet of the test section, where the meniscus is initially held stationary. The optical slice (referring to the imaging plane location governed by geometric optics, detailed in section 3.5 is next located at the channel wall and the test section is then filled with water. The test section is pressurized with the nitrogen supply while the valve at the outlet is closed. The cameras are triggered (the macro-view camera triggers the HSC as soon as it begins recording) and subsequently the outlet valve 
is opened. The image data from both cameras is saved. Next, the objective lens is moved upward $(0.5-1 \mu \mathrm{m}$ z-axis locations near the thin film interface and $1-2 \mu \mathrm{m}$ near the thick film interface) and the process is repeated. With this procedure, images of the film interface are gathered ranging from the channel wall to approximately $60 \mu \mathrm{m}$ above the channel wall.

\subsection{Image Processing}

Images are captured continuously as water is driven out of the test section by gas. For this reason, the images of interest must be identified. The macro-view camera captures at 30 frames per second (FPS), generally capturing one image of the meniscus before it passes the confocal view field and one image after. The location of the meniscus and of the trailing ridge are determined with Spotlight, an image analysis software developed by NASA [35]. The images are scaled with a known dimension,

and time/position coordinates of the areas of interest are output. Since both cameras have the same time 0, the absolute time of each image can be obtained. Offsetting the time so that the leading meniscus frame is time 0 , a plot of the meniscus location can be generated. Based on the location of the meniscus in the macro-view and the location of the objective lens in the macro-view, a plot of meniscus location relative to the confocal view imaging rate can be generated, as illustrated in figure 3.9 .

It can be readily observed that the contact line region (and therefore ridge) move more slowly than the meniscus. By plotting the location of the meniscus and ridge relative to the confocal view frame rate, images of the thick and thin film regions crossing the confocal view field can be selected for processing. This process is only 


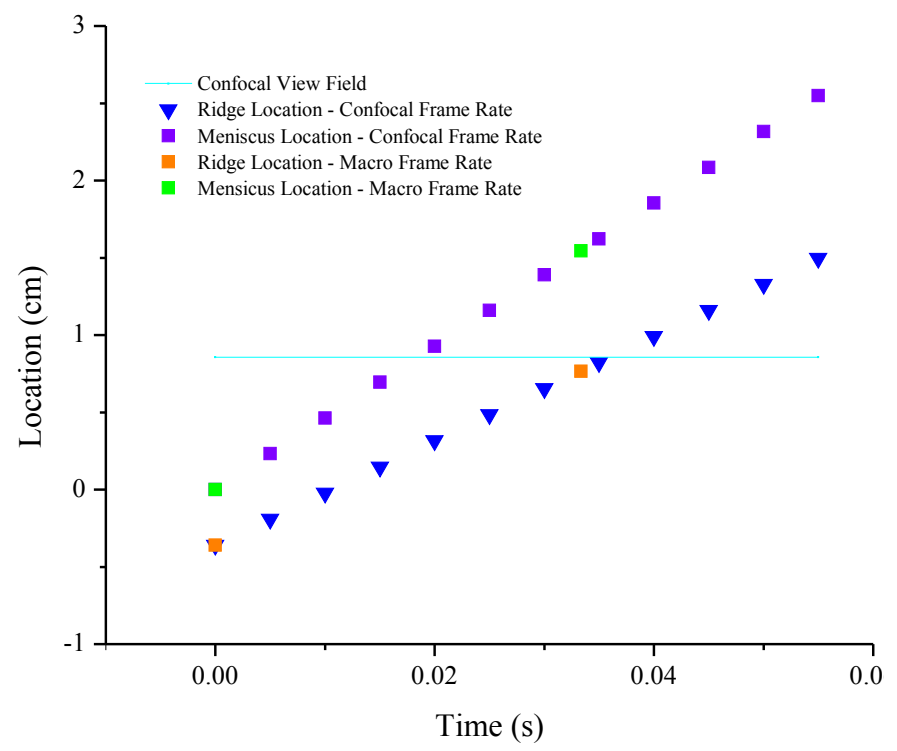

Figure 3.9. Location of plug-annular features relative to the macro-view camera frame rate and the confocal view frame rate

required for test runs with the Jai macro-view camera. No interpolation was required with the Photron HSC as the macro-view camera, because macro-view images were gathered at twice the rate of confocal images.

Thick and thin film regions exist within both the plug-annular region and within each discrete bubble. By measuring the bubble diameter (in pixels) in the thick and thin film regions, it was determined that the film thicknesses are equal for both the thick and thin film regions within the plug-annular film and bubble film. Several test runs were analyzed, and a representative image is shown in figure 3.10 .

This measurement method is not suitable for determining actual film thicknesses because of the severe optical distortion caused by the channel wall. Considering the distortion is equal within each image, an equal distance measured in pixels correlates with an equal physical distance; the film thicknesses must then be equal within the 


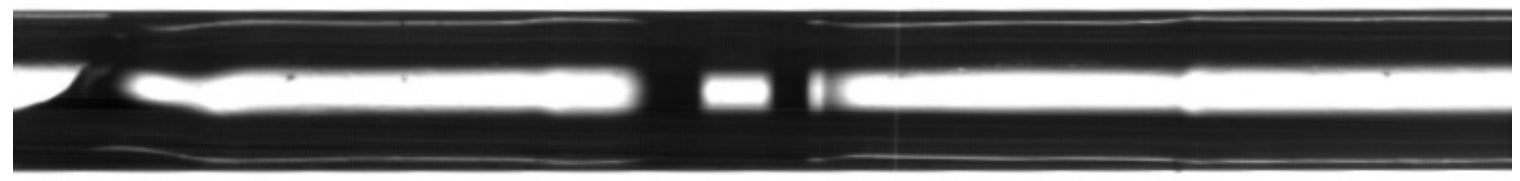

Figure 3.10. The thick and thin film thicknesses appear to be equal, allowing several images to be processed from each test run

uncertainty of the pixel-measurement technique. Multiple images from thick and thin film regions may then be extracted from the image data of each test run, which allows for a larger sample of intensity data for each objective lens position, increasing the certainty in intensity data for a given position.

The film thickness measurement technique used here is based on the mapping the reflection intensity of the film interface. The optical slice location is controlled with the objective lens location. Images are gathered with at optical slice locations ranging from below the film interface to above the film interface, as illustrated in figure 3.11

Interface reflection intensity reaches a maximum when the optical slice is located at the film interface, as illustrated in figure 3.12 B. Reflection intensity is low when the optical slice is located below the film interface, shown by figure 3.12 C. A split reflection is generated when the optical slice is located above the film interface, shown by figure 3.12 A.

The selected images are processed with a MATLAB code co-developed with [27] and used for [28]. Test runs outside the $C a$ range of interest, test runs which contained excess noise, and test runs which did not capture the film interface are not processed. A smoothing function is applied to the images to reduce noise due to fringe patterns in the reflection images. The selected images are cropped to a size selected by the user to analyze the intensity of the flattest part of the optical slice and to eliminate 

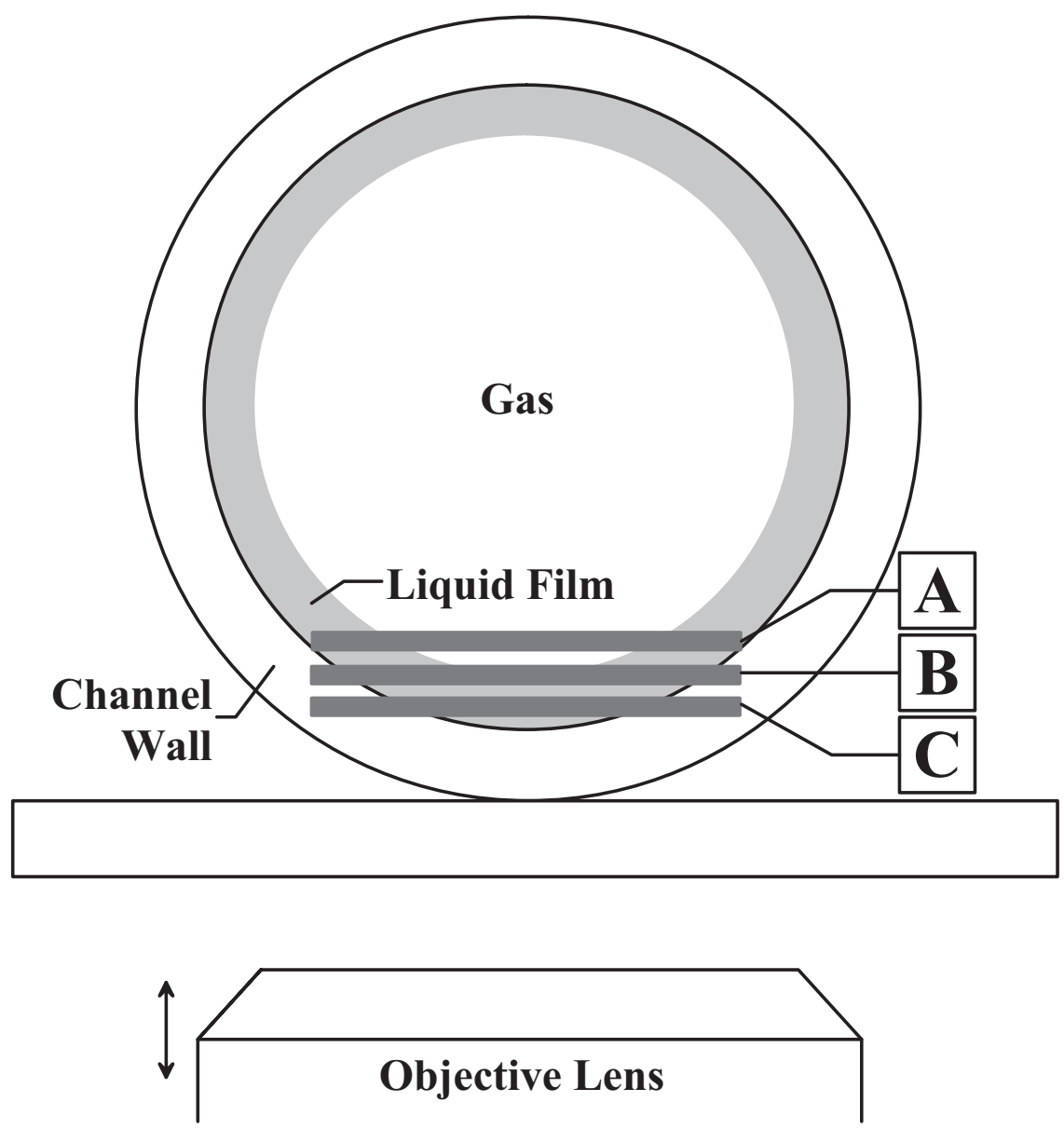

Figure 3.11. Intensity profiles at multiple optical slice locations: the optical slice positioned above the film interface (A), at the film interface (B), and below the film interface $(\mathrm{C})$ 

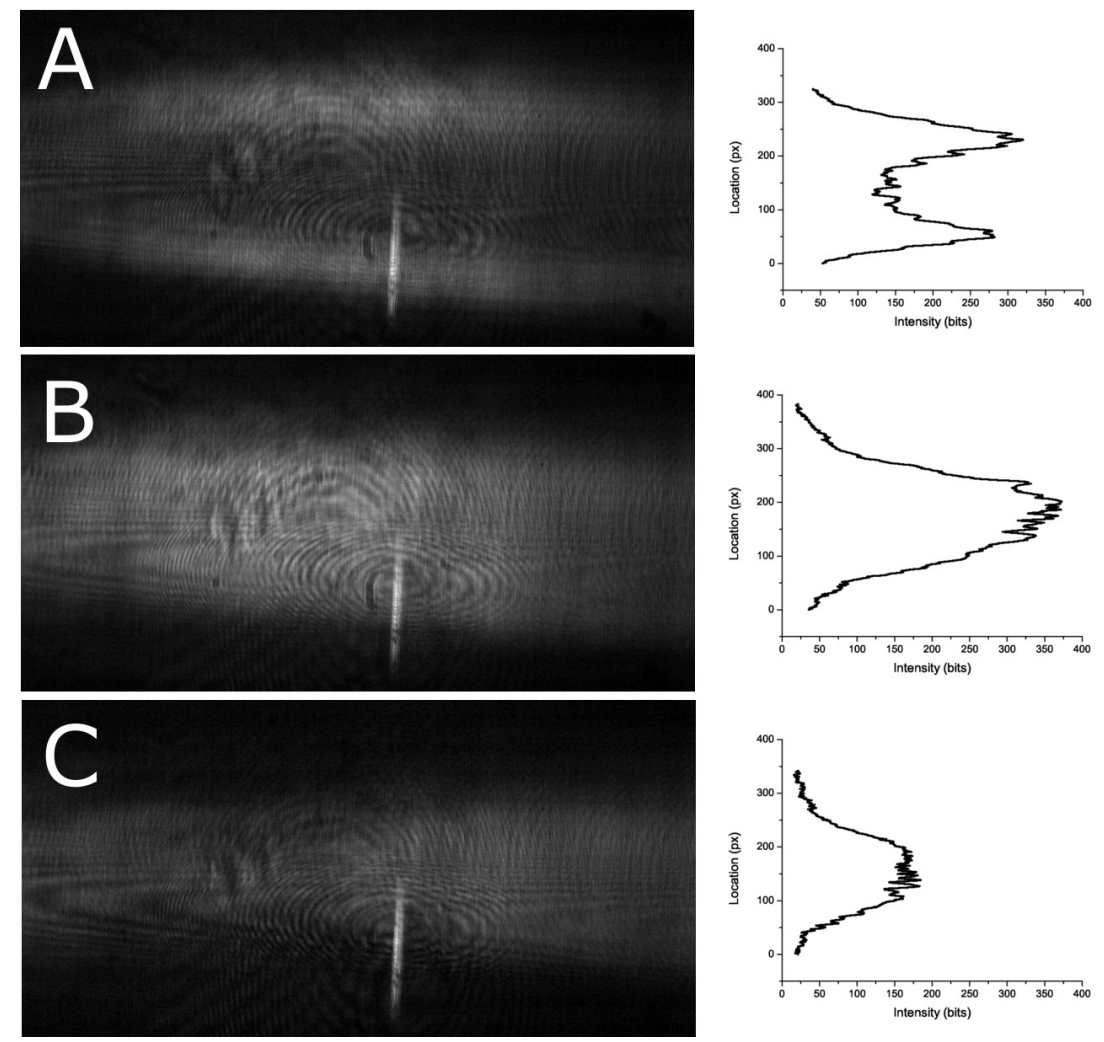

Figure 3.12. Location of plug-annular features relative to the macro-view camera frame rate and the confocal view frame rate 


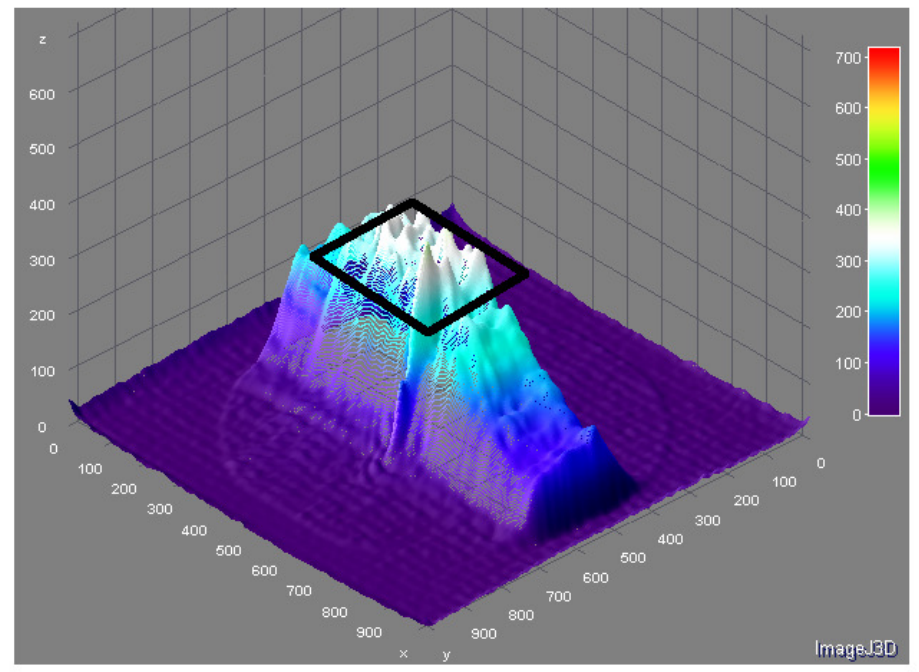

Figure 3.13. Intensity profile of an image with the optical slice located on the film interface

intensity data which may skew the average. Figure 3.13 is an intensity plot of an image with the optical slice located at the film interface.

Each image is cropped to the sized illustrated. This image size contains approximately the highest $5 \%$ of reflection intensity for an image gathered with the optical slice located at the film interface. Cropped to this size, the images contain information from the flattest part of the optical slice. In addition the intensity peaks generated with the optical slice located above the interface (figure 3.12 A) are eliminated.

The cropped images are then averaged across all pixels to generate an intensity value for each optical slice location. The intensity is then plotted as a function of z-axis location. The Lorentz function represents the intensity of a focused laser beam 
[49], so a Lorentz curve fit in OriginPro is used to precisely determine the peak reflection intensity location. The peak location combined with the location of the channel wall indicates the change in location of the objective lens. This distance is then correlated with the change in optical slice location via geometric optics.

\subsection{Focus Depth Correction}

Changes in refractive index along the beam path cause refraction at each change in optical medium. For this reason, a change in the objective lens position does not translate to the exact same change in optical slice position. Geometric optics predict the location of the optical slice based on the objective lens position. Snell's law determines the angle of refraction $\left(k_{2}\right)$ by the refractive indices the material $\left(n_{1}, n_{2}\right)$

on each side of the interface and the angle of incidence $\left(k_{1}\right)$, as shown in equation 3.3 .

$$
n_{1} \sin k_{1}=n_{2} \sin k_{2}
$$

The beam path into and out of the test section is illustrated in figure 3.14. The acceptance angle of the objective lens $\left(17.46^{\circ}\right.$ for the $10 \times$ objective and $26.76^{\circ}$ for the $20 \times$ ) is assumed to be the angle at which light enters and exits the planar bottom surface of the test section. The angle of refraction is calculated at each interface; angle measures with a prime (') indicate the reference is the normal vector of the curved channel wall. Between the planar bottom and the channel wall-water interface, the beam path is assumed to be perfectly linear as the media though which it travels (borosilicate and glycerol) are refractive index matched to three decimal points, $n_{s}=$ 1.474. The beam path is refracted at the channel wall-water interface; the refractive 


\section{Channel End View Channel Side View}
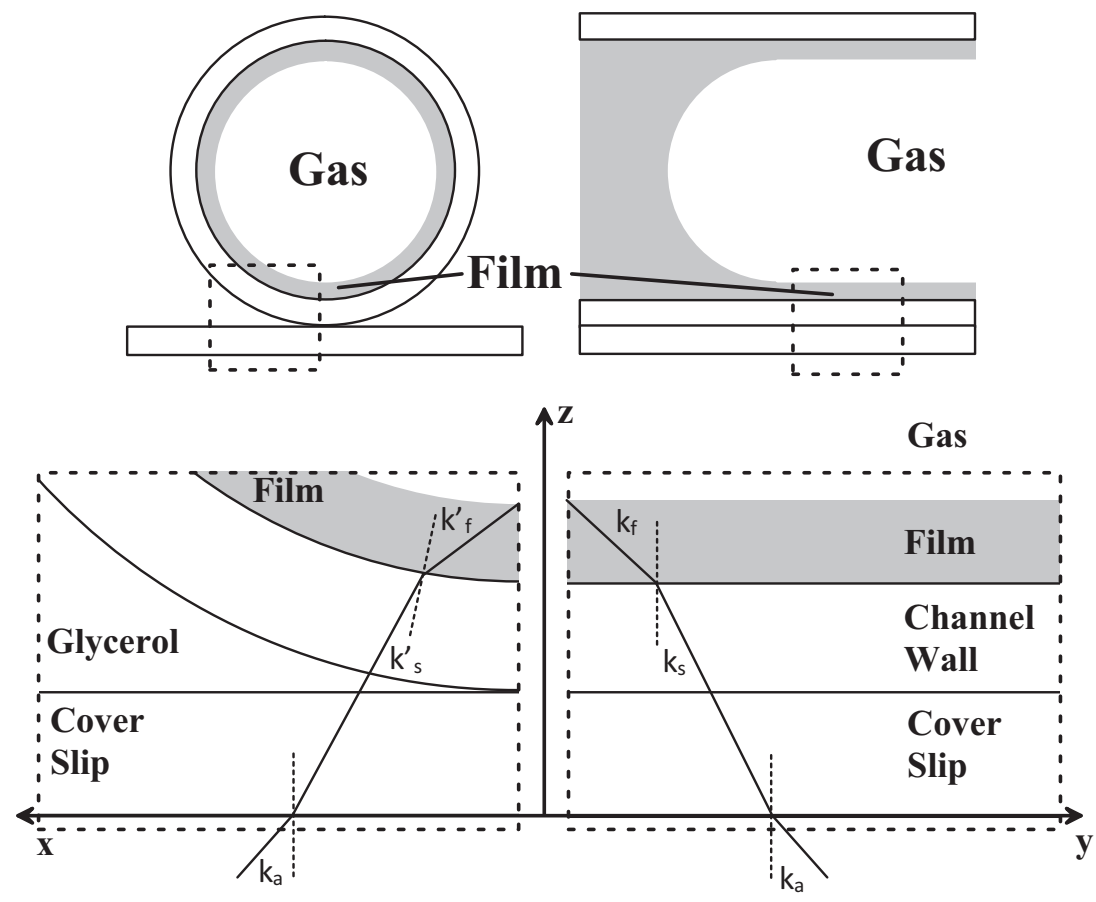

Figure 3.14. Beam path into the test section, as determined by Snell's Law 
index of water is assumed to be $n_{f}=1.33$.

A number of methods for developing a relation between the objective lens location and the optical slice locations exist. The small angle approximation is commonly used to simplify the calculation, but induces error. The method chosen here was graphical plotting and geometric relations in SolidWorks, a solid modeling software. The geometry shown in figure 3.14 was drawn to scale and Snell's law (without the small angle approximation) was constrained to the normal vector at each change in beam path. The virtual objective lens was then moved in increments, and the location of the optical slice was determined based on the curved channel wall geometry and linear channel wall geometry. The average of the correlation values is then the final correlation value. A linear correlation (linear because of the small change in location compared to the curvature of the channel wall) of 1.377 was found for th $10 \times$ objective lens, and 1.425 for the $20 \times$ objective lens. This method also allows for changing $\theta_{a}$ to test the assumption that the acceptance angle of the objective lens is an appropriate value to determine the optical slice location.

\subsection{Uncertainty}

The primary causes of uncertainty with this film thickness measurement technique are from Capillary number fluctuations between test runs, optical slice step size, and reflection intensity uncertainty generated by the combination of the illumination laser beam waist and camera bit-depth. The uncertainty in Capillary number is taken as the range observed within the number of test runs required to generate a film thickness measurement. The uncertainty due to optical slice step size is taken as 
half the distance between consecutive steps. The z-axis focus motor is repeatable to $0.01 \mu \mathrm{m}$, making uncertainty in the z-axis location negligible.

Basic geometric optics indicates that a focused laser beam (such as the one used for confocal illumination) crosses itself at infinitely focused point. In reality, the beam generates a "waist" around at the point it crosses itself, which is sometimes referred to as a Gaussian beam. The intensity of the beam reaches its axial maximum at the waist, and the radial maximum is at the axis of the beam waist. The radius of the beam along the axis is [15]

$$
r_{b}=\frac{\lambda}{\pi k_{b}}\left[1+\left(\zeta k_{b}\right)^{2}\right]^{1 / 2}
$$

where $\lambda$ is the wavelength of light in the medium the waist is generated in (water in this case), $k_{b}$ is the angle of beam convergence, and $\zeta$ is the axial location within the beam, with $\zeta=0$ at the beam waist. The wavelength of light in water is found with

$$
\lambda=\frac{\lambda_{0}}{n_{f}}
$$

where $\lambda_{0}$ is the wavelength of the beam in a vacuum (488nm) and $n_{f}$ is the refractive index of water. For this case $\lambda=367 \mathrm{~nm}$. The time-averaged normalized intensity distribution, a Lorentzian intensity profile along the beam axis, is described by [49]

$$
I\left(r_{b}, \zeta\right)=\frac{2}{\pi r_{w}^{2}} e^{2\left(r_{b} / r_{w}\right)^{2}}
$$

The change in intensity detectable with a 10-bit imaging device, such as the HSC attached to the RSFC, is limited by the bit depth. The intensity change detection limit is $\left(2^{10}-1\right) / 2^{10}=0.9990$. Using the intensity value of $0.9990 I(0,0)$, the distance 


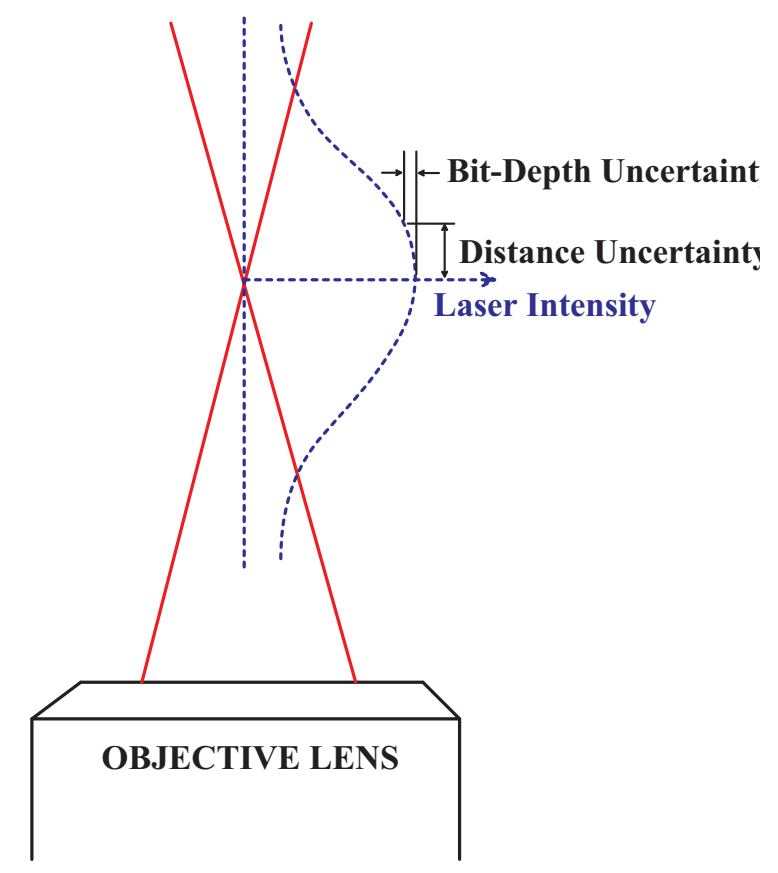

Figure 3.15. Illustration of bit depth uncertainty combined with laser intensity profile

$(\zeta-0)$ between two points of sufficient intensity change for detection can be found. The bit depth limitation then correlates to a physical distance about the beam focal point, as illustrated in figure 3.15 .

The uncertainty in intensity detection with the $10 \times$ objective is $\pm 0.10 \mu \mathrm{m}$ and $\pm 0.07 \mu \mathrm{m}$ with the $20 \times$ objective. The sum of all uncertainties is then the total uncertainty in film thickness measurement. 



\section{EXPERIMENTAL}

\subsection{Test Section Properties}

\subsubsection{Refractive Index}

Initially, fluorinated ethylene propylene (FEP) tubing was used for the working capillary of the experimental test section. The primary advantage of the polymer is a refractive index $\left(n_{F E P}=1.341\right)[41]$ which nearly matches that of water $\left(n_{\text {water }}=1.33\right)$. With nearly matching refractive indices, refraction and reflection at the channel wallwater interface are minimized. Minimized refraction means a geometric optics correction factor based on the curved channel wall-water interface may have been small enough to neglect. Less reflection at the channel wall-water interface allows more power to be transmitted into and out of the test section, providing increased reflection intensity at the film interface. Rain-X®coated borosilicate capillaries have $n_{\text {borosilicate }}=1.474$, making the optical properties of this type of test section less desirable. 


\subsubsection{Contact Angle and Surface Properties}

The shock condition has only been observed in non-wetting capillaries, so the capillary test section must have a high contact angle. FEP capllaries are non-wetting with $\theta \cong 95^{\circ}$, as are Rain-X®coated borosilicate capillaries with $\theta \cong 105^{\circ}$ [24].

The use of FEP test sections was ultimately abandoned due to surface imperfections along the interior of the channel wall, likely caused by the manufacturing process of the plastic tubing. The surface imperfections consisted of helical scratches of various length along the interior of the tubing. The helical scratches were observed to affect the symmetry of the contact line about the axis due to contact line pinning upon contact with a scratch.

\subsection{Initial Observations}

Although the interior surfaces of the FEP capillaries were non-uniform, behavior similar to that observed by [24] with Rain-X®coated borosilicate capillaries was

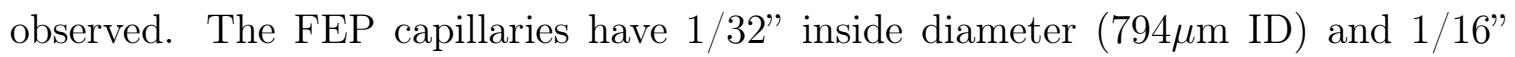
outside diameter. Figure 4.1 illustrates flow morphology in the plug-annular region at a speed below the critical Capillary number. Similar to [24], no shock is observed at low speeds in a non-wetting channel.

Exceeding the critical Capillary number detailed by [24], the shock is observed with FEP test sections, shown in figure 4.2. Capillary number is not the only factor affecting film morphology produced by driving liquid out of a capillary with gas. The existence of two gas bubbles near entrance of the, otherwise liquid-filled, capillary generated long Taylor bubbles in the flow. The length of the bubbles allowed 

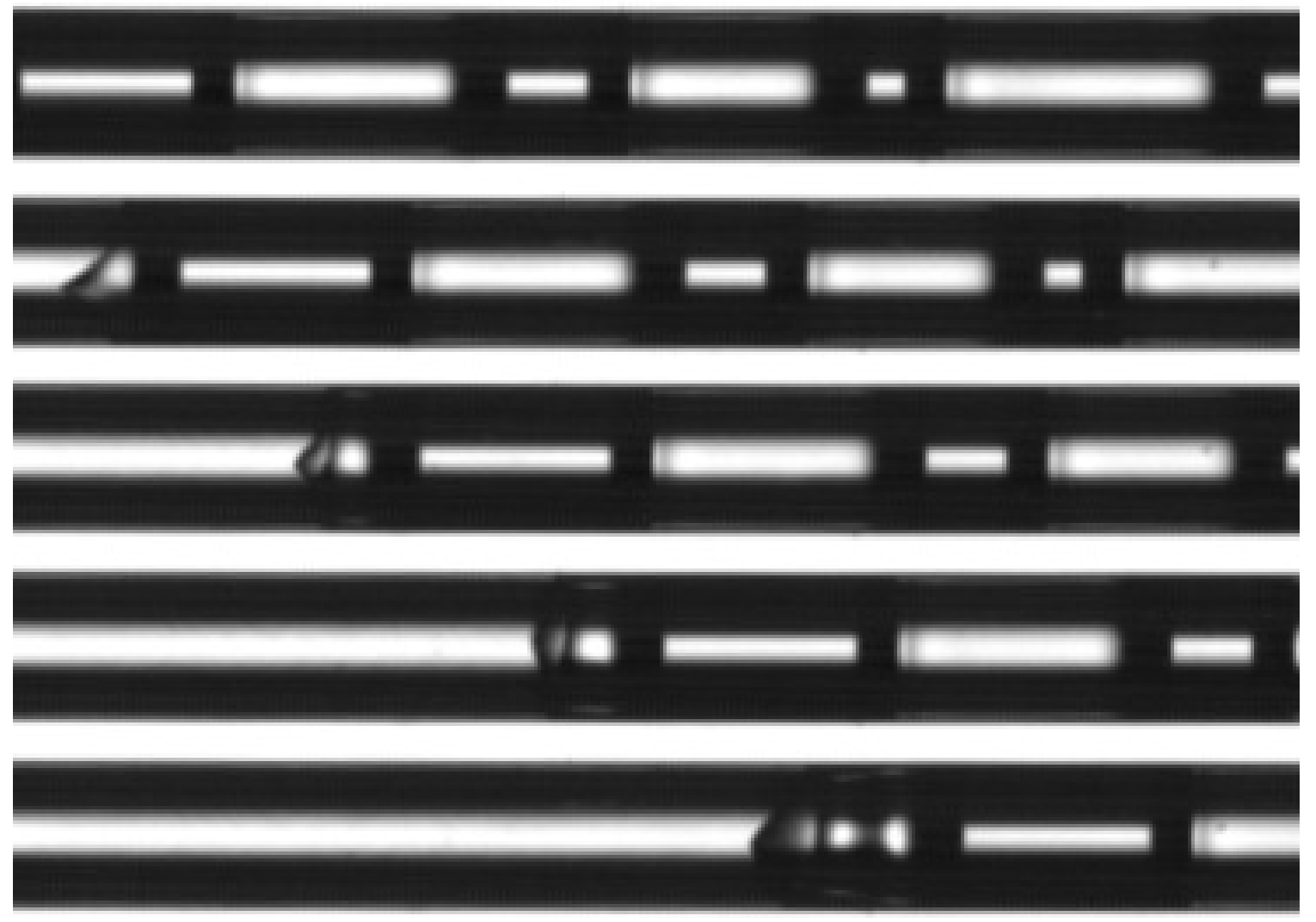

Figure 4.1. No shock is observed with film evolution of plug-annular region of FEP test section with $C a=0.00353$. The image sequence is with $10 \mathrm{~ms}$ between frames and the channel length shown is $14.5 \mathrm{~mm}$. 
instabilities to grow, which ultimately lead to film rupture, as illustrated in figure 4.3 .

Upon film rupture, the non-wetting substrate causes the film to dewet the surface. As dewetting occurs, the contact line moves toward the nearest plug. As the contact line moves, the film continues rupturing, which deposits drops on the channel wall. The shock phenomena was observed somewhat consistently in the Taylor bubbles before film rupture.

The robustness of the shock phenomena is demonstrated by the fact that it appears in adverse conditions, such as with rough channel walls and within long Taylor bubbles. The optical properties of FEP and the natural contact angle of $\sim 95^{\circ}$ make FEP an attractive material for this experiment; if FEP tubing with very smooth channel is available, it may be a useful material for future experimentation.

Test runs with $500 \mu \mathrm{m}$ ID Rain-X®coated borosilicate capillaries were used for all quantitative film thickness measurement experiments. The highest speed test run used for film thickness measurement had an average $C a=0.01015$. This test run, with relatively large magnitude viscous forces compared to surface tension, showed unique behavior in the thick film region, shown in figure 4.4 .

The high speed of the test run caused the length of the thick film region to decrease when the ridge pinches off to form a plug, shown in image 4 to 5 of figure 4.4 . The short length of the thick film region and the high velocity of the flow precluded consistent image capture with the confocal unit at 200 frames per second. The thin film region was imaged consistently for $C a=0.01015$, allowing for comparison of the thin film measurement to the theory. At even higher speeds, with $C a=0.0158$, a departure from uniform film thickness in the thick film region was also observed and is illustrated in figure 4.5 .

With $C a=0.0158$, a dimple appears between the capillary ridge and the thick 

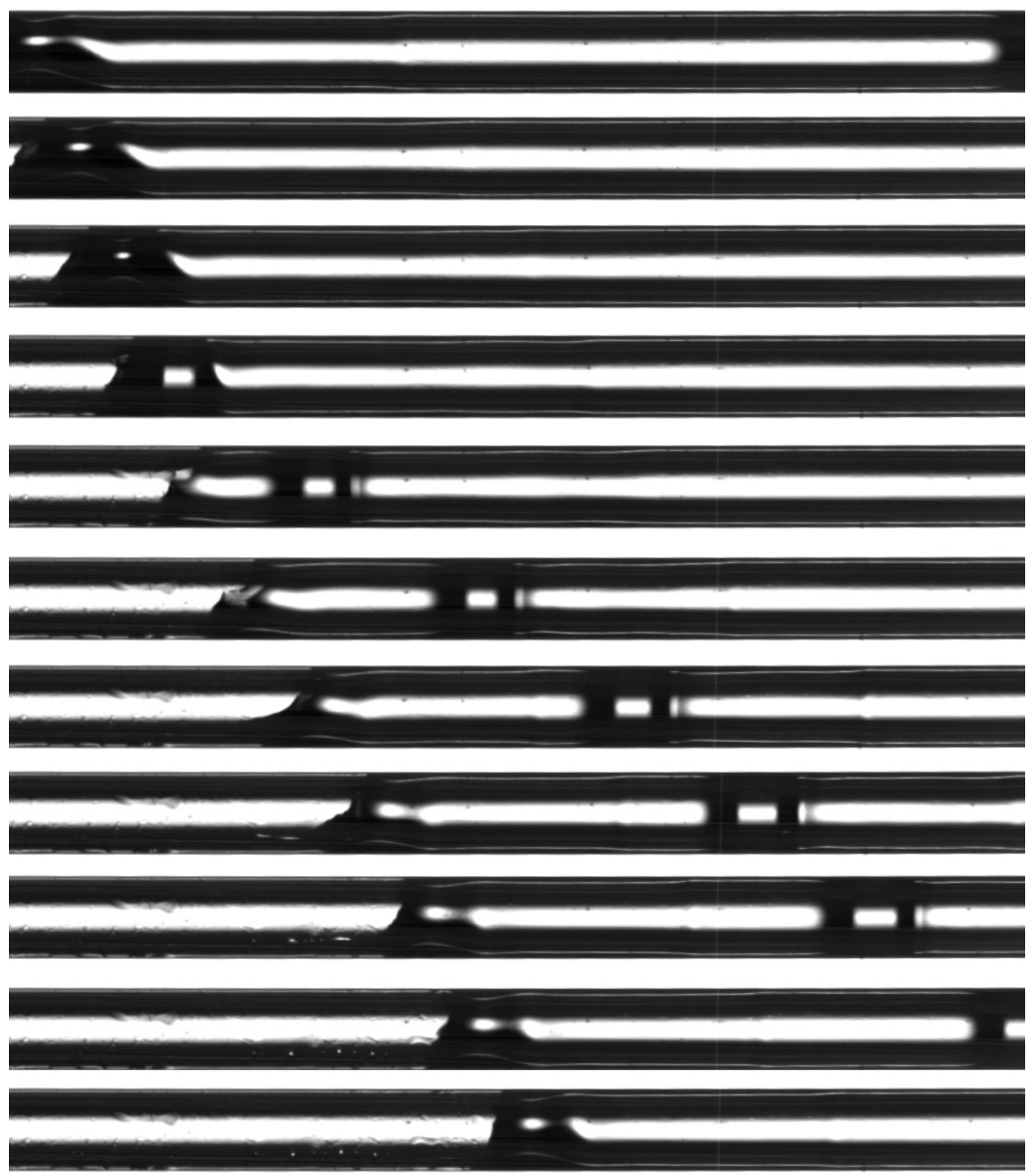

Figure 4.2. Shock structure formation in plug-annular regime with $C a=0.0172$. Image sequence is with $2 \mathrm{~ms}$ between frames and the channel length shown is $20 \mathrm{~mm}$. 


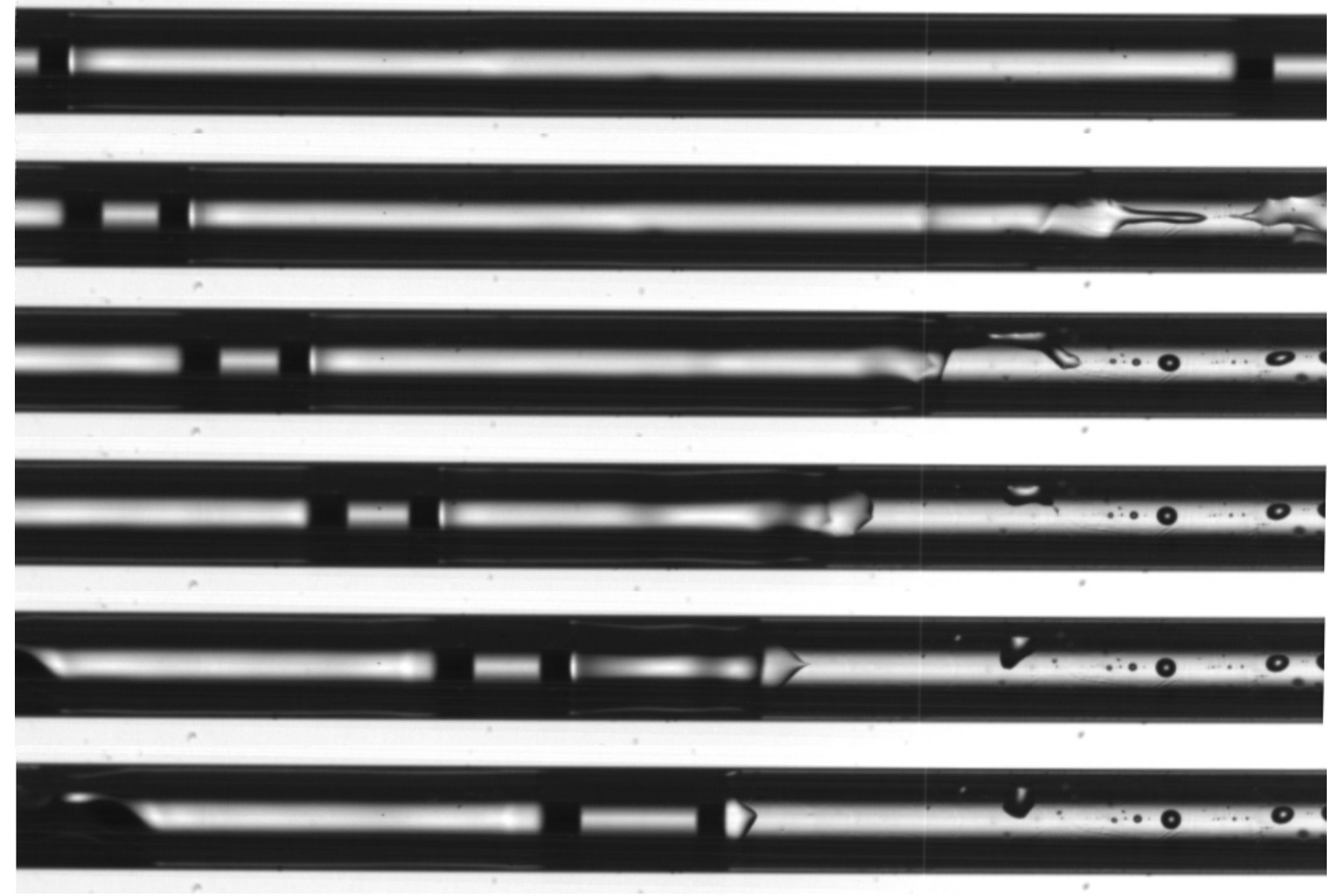

Figure 4.3. Film rupture in a non-wetting Taylor bubble due to Rayleigh instabilities with $C a=0.00668$. The image sequence is with $4 \mathrm{~ms}$ between frames and the channel length shown is $20 \mathrm{~mm}$. 

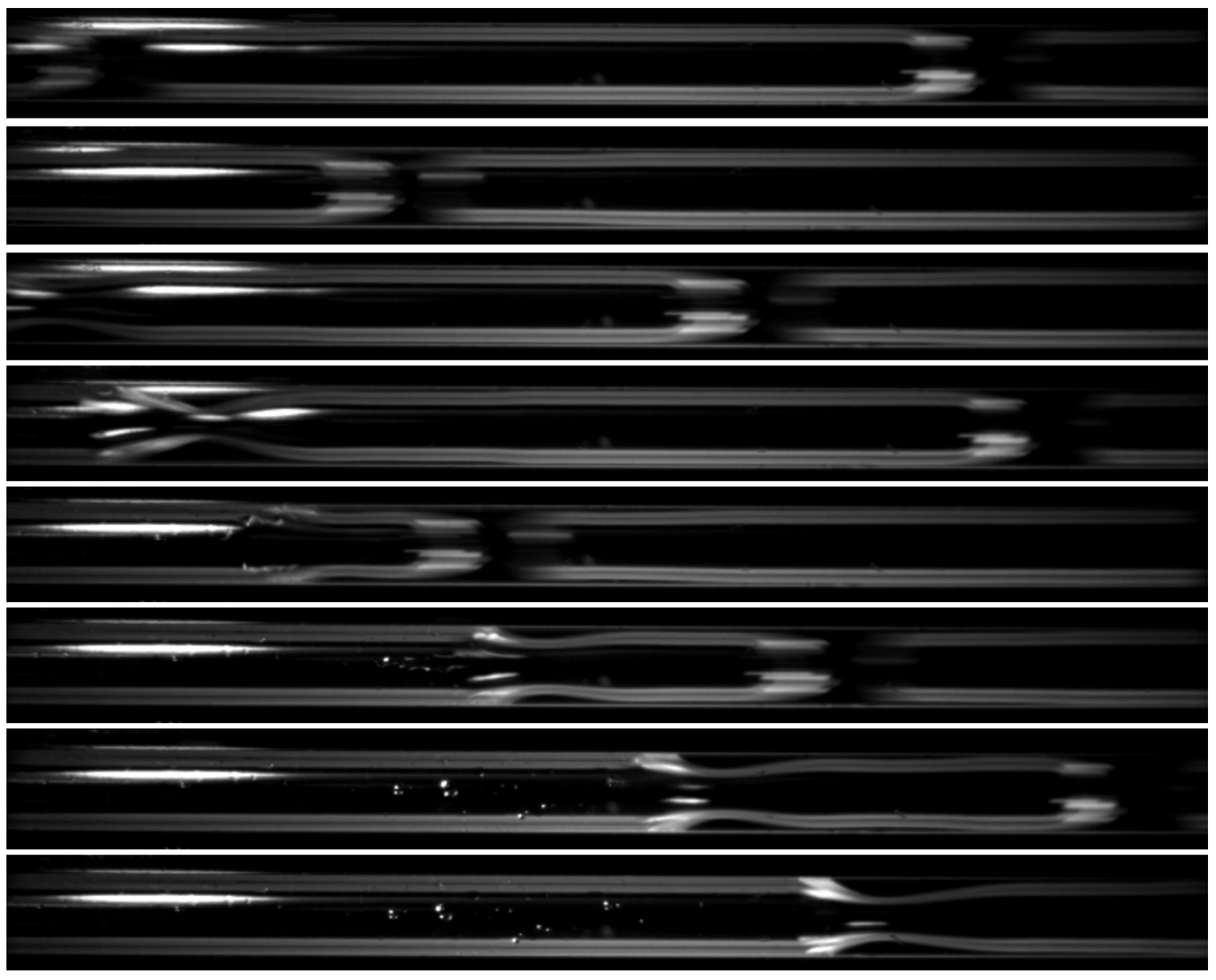

Figure 4.4. Location of shock rapidly changes after pinch off (image 4 to 5 ) with $C a=0.01015$; thick film morphology may change in Taylor bubbles at high speeds. The image sequence is with $5 \mathrm{~ms}$ between frames and the channel length shown is $8.5 \mathrm{~mm}$. 


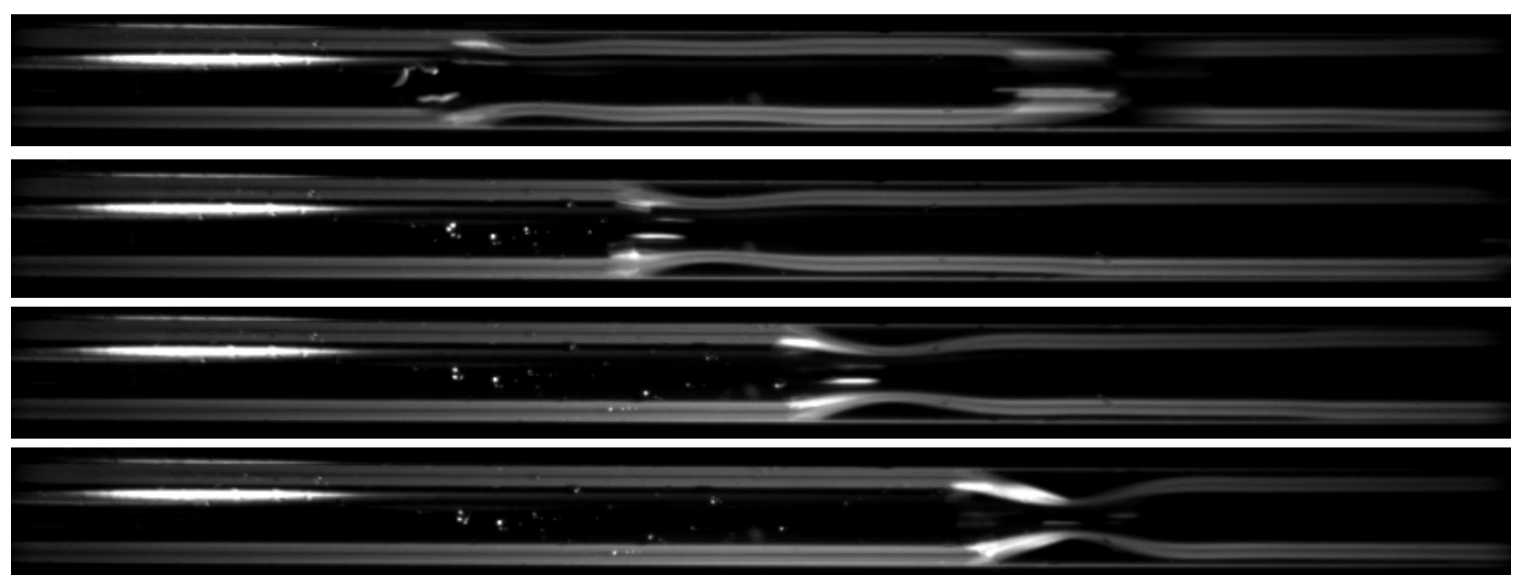

Figure 4.5. Film morphology becomes more dynamic and unstable at $C a=0.0158$. The image sequence is with $5 \mathrm{~ms}$ between frames and the channel length shown is $8.5 \mathrm{~mm}$.

film region. The non-uniformity in film thickness and relative instability of thick film precluded thickness measurement in the thick film region with confocal microscopy. At a $C a$ of 0.0158 , the $W e$ is 5.4 , indicating the inertial forces influence film morphology. Rapid contact line motion also causes the contact line to rupture, depositing drops on the channel wall, and inducing additional dynamics which may affect film morphology.

\subsection{Film Thickness Measurement Experimental Results}

The first step in film thickness measurement is locating the channel wall relative to objective lens location (z-axis). To do this, images are captured at optical section locations ranging from below the dry channel wall to above the dry channel wall. Location of the channel wall gives a reference point on the z-axis coordinate system. Next, the liquid-air interface is located in the z-axis reference frame for both the thick and thin film. The difference between the channel wall location and the film 


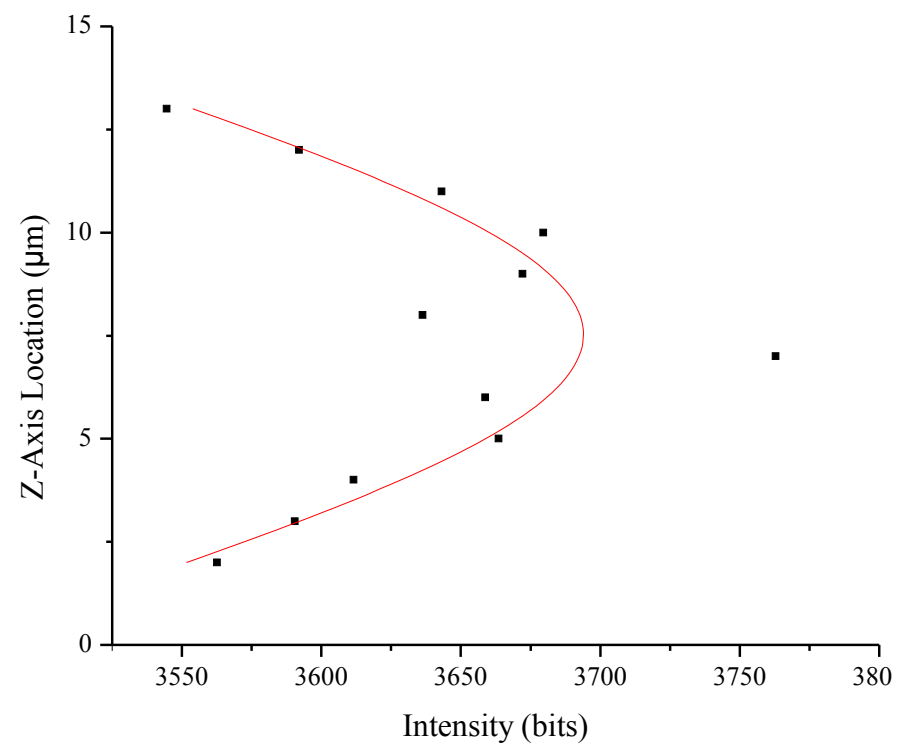

Figure 4.6. Reflection intensity of channel wall-air interface as a function of z-axis location for test runs with $C a=0.00702$

interface location gives the 'apparent film thickness.' The apparent film thickness is then multiplied by the geometric optics correction factor (determined in section 3.5) to determine the actual film thickness.

The channel wall location is determined by the reflection intensity at various zaxis locations. Figure 4.6 illustrates a Lorentz curve fit of the image intensities used to locate the channel wall.

Once the channel wall is located, test runs with dynamic liquid films can begin. The channel wall location is used as a starting point to gather image data at z-axis locations stepping upward through the film. Image data is continuously captured at a stationary z-axis location as water is driven out of the test section. The z-axis location is then changed and image data is continuously captured as water is driven out of the test section. This means each z-axis location requires a separate test run. 
Images of the thin film interface at each z-axis location are isolated by interpolating the macro-view images (detailed in section 3.4). The image intensity as a function of optical slice location follows a similar trend to the discussion developed in section 3.4. The intensity data for the film thickness measurement with $C a=0.00702$ was gathered with the $10 \times$ objective lens. This lens size means the optical slice is approximately $86.6 \mu \mathrm{m}$ thick. The thick optical slice relative to the film thickness produces image intensities which include the reflection from the channel wall as well as the gas-liquid interface, producing a fading image intensity for optical slice locations moving away from the channel wall. For test runs with $C a=0.00419$ and $C a=0.01015$, the $20 \times$ objective lens was used. The much thinner optical slice thickness $(9.84 \mu \mathrm{m})$ of the $20 \times$ objective removes much more light from the channel wall reflection. The intensity data gathered (for all optical slice locations) with the $10 \times$ objective lens in the thin film region is illustrated in figure 4.7 .

The image data in the vicinity (z-axis) of the thin film interface is then isolated and fitted with a Lorentzian curve, shown in figure 4.8. The peak of the Lorentz fit indicates the z-axis location of the thin film interface.

The process is then repeated for images of the thick film interface. The intensity profile as a function of z-axis location for the thick film is illustrated in figure 4.9 .

The peak intensity of the thick film interface is lower, as expected, because less light reflected from the channel wall is captured in the thickness of the optical slice. The intensity data as a function of z-axis location is again isolated and a Lorentz curve is fitted, shown in figure 4.10 .

The apparent film thickness is the difference between the peak intensity of the channel wall and the peak intensity of each film interface. The apparent film thickness is then multiplied by the geometric optics correction factor discussed in section 3.5 . 


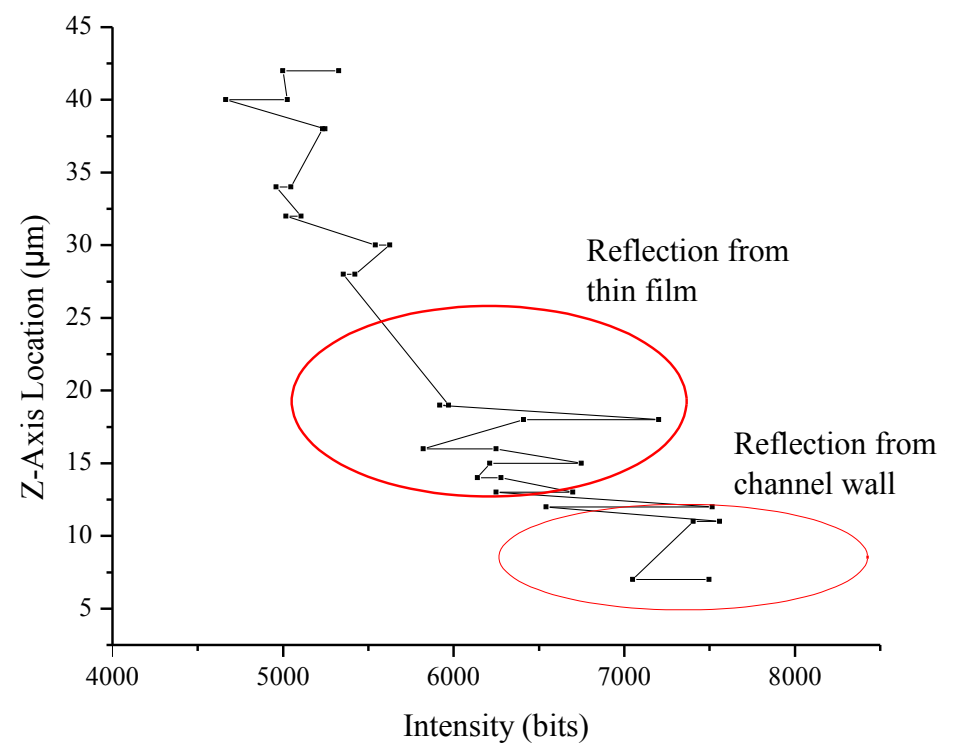

Figure 4.7. Reflection intensity of thin film-air interface as a function of z-axis location, $C a=0.00702$

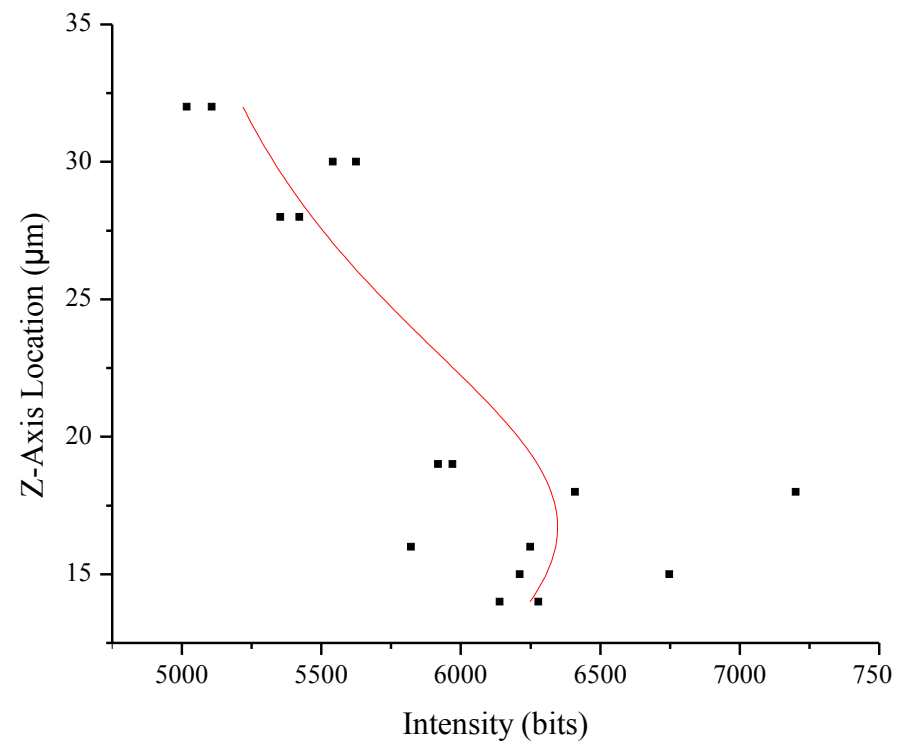

Figure 4.8. Lorentzian fit of reflection intensity in vicinity of thin film-air interface, $C a=0.00702$ 


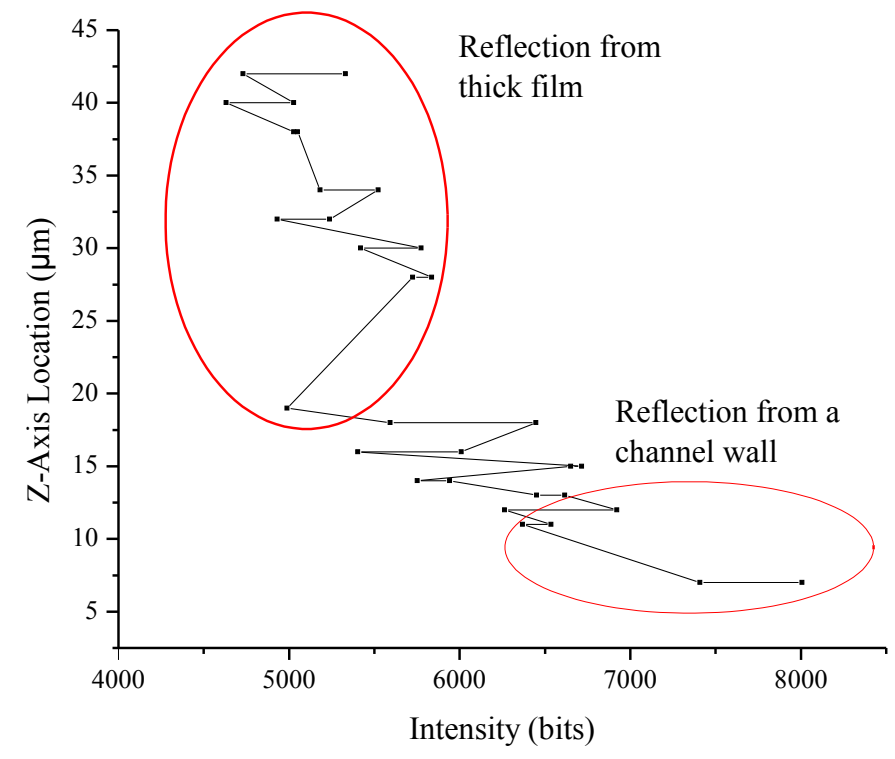

Figure 4.9. Reflection intensity of thick film-air interface as a function of z-axis location, $C a=0.00702$

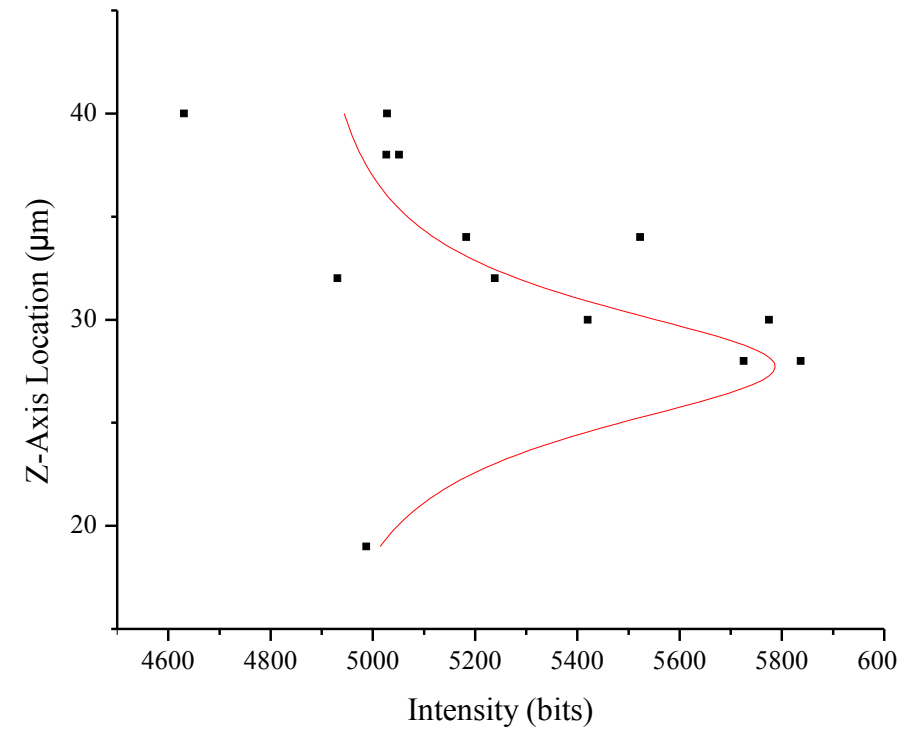

Figure 4.10. Lorentzian fit of reflection intensity in vicinity of thick film-air interface, $C a=0.00702$ 


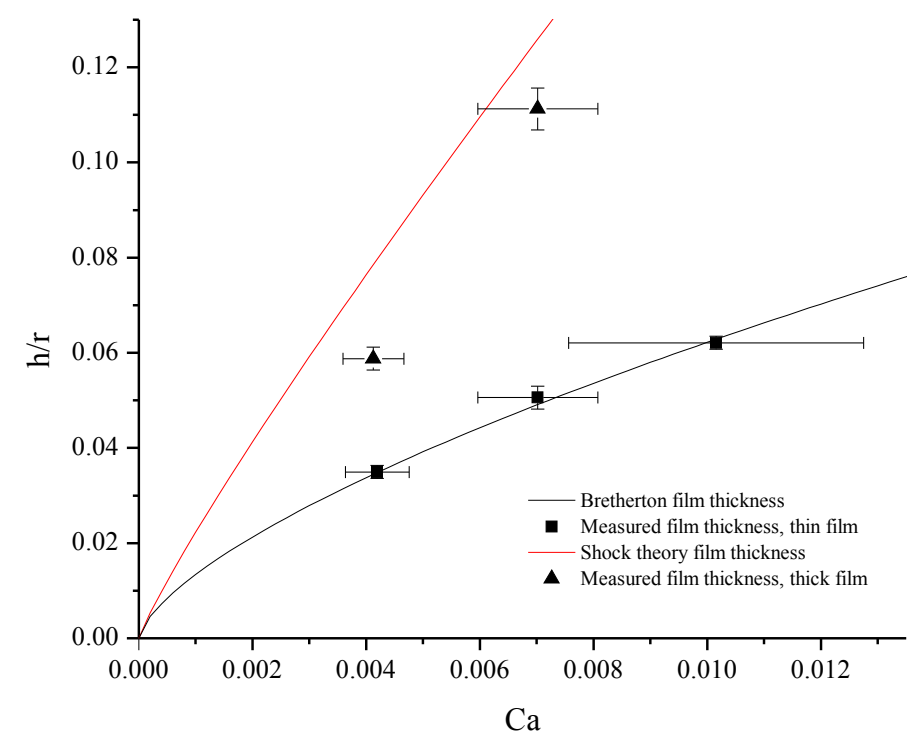

Figure 4.11. Film thickness as a function of Capillary number compared to film thickness model developed by [24]

The actual film thickness is then nondimensionalized by the channel radius $(\mathrm{h} / \mathrm{r})$, and plotted as a function of Capillary number, shown in figure 4.11. The uncertainty discussed in section 3.6 is included with vertical and horizontal error bars.

The thickness of the thin liquid film region appears to be in good agreement with the Bretherton correlation, within the uncertainty in $\mathrm{Ca}$. The assumptions made in the derivation of the Bretherton relation limit its applicability to $\mathrm{Ca}<10^{-3}$ [8], but the uncertainty is less than the repeatability of the experimental $C a$. It should also be noted that the Bretherton correlation assumes $\theta=0$.

The thick film theory developed by [24] appears to over predict the film thickness in the thick film region. Factors which may affect the thick film thickness include static contact angle, dynamic contact angle, and channel diameter. The contact angle must play a role in the formation of the shock because with $\theta=0$, no shock 
is generated in the film. The contact angle may also affect the thickness of the thick film region. The channel diameter may also affect the film thickness in the thick film region. It has been shown that azimuthal (perpendicular to the direction of flow) curvature affects film thickness and morphology on non-wetting substrates [9].

The thick film theory developed by [24] is a semi-empirical relation which uses film thickness data which may not be accurate. The relation could be improved by using a more precise method to measure film thickness, which would make the correlation more precise. Finally, the model contains the implicit assumption that the system is steady-state. The experiment is based on flushing liquid out of a capillary, which requires the liquid to start from rest and accelerate through the length of the channel. This acceleration likely has an effect on film thickness.

The accuracy of the confocal film thickness measurement technique employed here could be greatly improved by increasing the $C a$ repeatability for each test run. An increased rate of confocal imaging, and therefore a more representative sample of interface reflection intensity, could also increase the regularity of reflection intensity profiles and therefore the accuracy of the measurement technique. 


\section{Conclusions}

Surface tension forces are significant at millimeter length-scales, causing profoundly different flow morphologies in microchannels than in macroscale flows. The existence and morphology of thin liquid films is particularly relevant for predicting performance and operational stability of devices containing microscale two phase flows. Film deposition on a perfectly wetting flat substrate is one of the earliest studies of film thickness. The plug flow regime has been studied extensively analytically, computationally, and experimentally. The plug-annular flow regime has been studied primarily experimentally, likely due to the difficulty in modeling film surface reordering in this regime. Annular flows have been studied analytically, computationally, and experimentally.

The effect of static contact angle on film thickness and morphology in microchannels is a topic that is not well understood. [24] observed a novel film morphology in microchannel plug-annular flow. The phenomena is referred to as a 'shock,' because a thin film jumps to a thicker film in a very short distance. A novel film thickness measurement technique is employed to better understand this novel film phenomena. By analyzing the reflection intensity of a film interface as a function of the objective lens location, film thickness can be precisely determined with confocal microscopy.

The thickness of the thin liquid film region appears to be in good agreement with 
the Bretherton correlation, within the uncertainty in $C a$. It should also be noted that the Bretherton correlation assumes $\theta=0$. The thick film theory developed by [24] appears to over predict the film thickness in the thick film region. Factors which may affect the thick film thickness include static contact angle, dynamic contact angle, and channel diameter. The accuracy of the model developed by [24] may be limited by the film thickness measurements used the derivation, as well as, the assumption of steady-state fluid flow, neglecting acceleration effects.

The scanning speed of the confocal microscope limits the speed at which film interfaces may be imaged, and therefore the number of images of a moving interface. Currently, the RSFC is limited to 200 frames per second, which allows for one image of the thick and thin film interfaces to be analyzed for each test run. An increased scanning speed would allow for multiple images of the thick and thin film interfaces to be averaged, and would therefore increase accuracy of the reflection intensity value. The accuracy of the film thickness measurement technique employed here could also be greatly improved by increasing the $C a$ repeatability for each test run. 


\section{REFERENCES}

[1] Sergey Alekeseenko, Andrey Cherdantsev, Okasana Heinz, Sergey Karlamov, and Dmitriy Markovich. Analysis of spatial and temporal spectra of liquid film surface in annular gas-liquid flow. Experiments in Fluids, 54:1590-1-1597-12, 2013.

[2] R. W. Aul. The Motion of Drops and Long Bubbles Through Small Capillaries: Coalescence of Drops and Annular Film Stability. PhD thesis, Cornell University, 1989.

[3] R. W. Aul and W. L. Olbricht. Stability of a thin annular film in pressure-driven, low-reynolds-number flow through a capillary. Journal of Fluid Mechanics, 215: $585-599,1990$.

[4] Pascale Aussillous and David Quéré. Quick depostion of a fluid on the wall of a tube. Physics of Fluids, 12(10):2367-2371, 2000.

[5] A. M. Barajas and R. L. Panton. The effect of contact angle on two-phase flow in capillary tubes. International Journal of Multiphase Flow, 19(2):337-346, 1993.

[6] A. Bazylak. Liquid water visualization in PEM fuel cells: A review. International Jounal of Hydrogen Energy, 34:3845-3857, 2009. 
[7] Lydéric Bocquet and Elisabeth Charlaix. Nanofluidics, from bulk to interfaces. Chemical Society Reviews, 39:1073-1095, 2010.

[8] F. P. Bretherton. The motion of long bubbles in tubes. Journal of Fluid Mechanics, 10:166-188, 1961.

[9] Tak Shing Chan, Thomas Gueudré, and Jacco H. Snoeijer. Maximum speed of dewetting on a fiber. Physics of Fluids, 23:112103, 2011.

[10] B. G. Cox. On driving a viscous fluid out of a tube. Journal of Fluid Mechanics, 14:6-14, 1962.

[11] R. G. Cox. The dynamics of the spreading of lliquid on a solid surface. part 1. viscous flow. Journal fo Fluid Mechanics, 168:169-194, 1986.

[12] Pierre-Gilles de Gennes, Francoise Brochard-Wyart, and David Quéré. Capillarity and Wetting Phenomena. Springer-Verlag New York, Inc., 2004.

[13] E. B. Dussan. On the spreading of liquid on solid surfaces: Static and dynamic contact lines. Annual Review of Fluid Mechanics, 11:371-400, 1979.

[14] Jens Eggers. Existence of receding and advancing contact lines. Physics of Fluids, 17:082106, 2005.

[15] Neil J. Everall. Modeling and measuring the effect of refraction on the depth resolution of confocal raman microscopy. Applied Spectroscopy, 54(6):773-782, 2000.

[16] Mara D. Giavedoni and Fernando A. Saita. The axisymmetric and plane cases of a gas phase steadily displacing a newtonian liquid - a simultaneous solution of the governing equations. Physics of Fluids, 9(8):2420-2429, 1997. 
[17] María D. Giavedoni and Fernando A. Saita. The rear meniscus of a long bubble steadily displacing a newtonian liquid in a capillary tube. Physics of Fluids, 11: 786-794, 1999.

[18] Alan R. Gibbs, Glen MacDonald, and Karl Garsha. Handbook of Biological Confocal Microscopy, Third Edition. Springer Science+Business Media, LLC, 2006.

[19] Yougbae Han and Naoki Shikanozo. Measurement of the liquid film thickness in micro tube slug flow. International Journal fo Heat and Fluid Flow, 30:842-853, 2009.

[20] Yougbae Han and Naoki Shikanozo. The effect of bubble acceleration on the liquid film thickness in microtubes. International Journal of Heat and Fluid Flow, 31:630-639, 2010.

[21] Youngbae Han and Naoki Shikanozo. Measurement of liquid film thickness in micro square channel. International Journal of Multiphase Flow, 35:896-903, 2009.

[22] Tatsuya Hazuku, Norihiro Fukamachi, Tomoji Takamasa, Takashi Hibiki, and Moamoru Ishii. Measurement of liquid film in microchannels using a laser focus displacement meter. Experiments in Fluids, 38:780-788, 2005.

[23] Matthias Heil. Finite reynolds number effects in the Bretherton problem. Physics of Fluids, 13(9):2517-2521, 2001.

[24] Alexandru Herescu. Two-Phase Flow In Microchannels: Morphology And Interface Phenomena. PhD thesis, Michigan Technological University, 2013. 
[25] Alexandru Herescu and Jeffrey S. Allen. The effect of surface wettability on viscous film deposition. In Proceedings of the 7th International ASME Conference on Nanochannels, Microchannels and Minichannels, page ICNMM2009, 2009.

[26] Alexandru Herescu and Jeffrey S. Allen. Implications of contact line dynamics on Taylor bubble flow morphology. In ASME 2010 3rd Joint US-European Fluids Engineering Summer Meeting and 8th International Conference on Nanochannels, Microchannels and Minichannels, Montreal, Canada, 2010.

[27] Joseph E. Hernandez. Development of Confocal Imaging Techniques for Probing Interfacial Dynamics in Microscale Gas-Liquid Two-Phase Flow. PhD thesis, Michigan Technological University, 2014.

[28] Joseph E. Hernandez, David C. Deisenroth, and Jeffrey S. Allen. Thickness measurement of dynamic thin liquid films in microchannels using reflectance swept-field confocal microscopy. Experiments in Fluids, in review, 2013.

[29] Volker Hessel, Holger Lốwe, and Friedhelm Schőnfeld. Micromixers - a review on passive and active mixing principles. Chemical Engineering Science, 60:24792501, 2004.

[30] Charles E. Hickox. Instability due to viscosity and density stratification in axisymmetric pipe flow. Physics of Fluids, 14(2):251-262, 1970.

[31] L. M. Hocking. Meniscus draw-up and draining. Euro. Jnl of Applied Mathematics, 12:195-208, 2001.

[32] Richard L. Hoffman. A study of the advancing interface: I. interface shape 
in liquid-gas systems. Journal of Colloid and Interface Science, 50(2):228-241, 1975.

[33] O. E. Jensen. The thin liquid lining of a weakly curved cylindrical tube. Journal of Fluid Mechanics, 331:373-403, 1997.

[34] Mark Johnson, Roder D. Kamm, Lee Wing Ho, Ascher Shapiro, and T. J. Pedley. The nonlinear growth of surface-tension-driven instabilities of a thin annular film. Journal of Fluid Mechanics, 233:141-156, 1991.

[35] Robert Klimek and Ted Wright. Spotlight8 image analysis software. Technical report, NASA/TM2006-214084, Glenn Research Center, Cleveland, Ohio, 21000 Brookpark Road, Cleveland, Ohio, January 2006.

[36] L. D. Landau and B.V. Levich. Dragging of a liquid by a moving plate. Acta physicochimica U.R.S.S., 17:42.54, 1942.

[37] Ying-Chih Liao, Yen-Ching Li, and Hsien-Hung Wei. Drastic changes in interfacial hydrodynamics due to wall slippage: Slip-intensified film thinning, drop spreading, and capillary instability. Physical Review Letters, PRL 111:136001, 2013.

[38] Mahshid Mohammadi and Kendra V. Sharp. Experimental techniques for bubble dynamics analysis in microchannels: A review. Journal of Fluids Engineering, 135:021202-1 - 021202-10, 2013.

[39] Wali M. Nozat. Measurement of liquid-film thickness by laser interferometry. Applied Optics, 36(30):7864-7869, 1997. 
[40] D. Quéré. On the minimal velocity of forced wetting in partial wetting. $C . R$. Acad. Sci. Ser. II: Mec. Phys. Chim. Sci., 313:313, 1991.

[41] Inc R. J. Chase Company. FEP handbook. 4000 Tara Court, Union City, CA 94587, U.S.A.

[42] John Ratuloski and Hsueh-Chia Chang. Transport of gas bubbles in capillaries. Physics of Fluids A, 1(10), 1989.

[43] C. Redon, F. Brochard-Wyart, and F. Rondelez. Dynamics of dewetting. Physical Review Letters, 66(6):715-719, 1991.

[44] Joseph M. Rodriguez, Onkar Sahni, Richard T. Lahey, and Kenneth E. Jansen. A parallel adaptive mesh method for the numerical simulation of multiphase flows. Computers \&f Fluids, 87:115-131, 2013.

[45] I. Schmidhalter, R. L. Cerro, M. D. Giavedoni, and F. A. Saita. Liquid film drag out in the presence of molecular forces. Physics of Fluids, 25(032105), 2013.

[46] R.S. Scott. Two-Phase Flow in Microchannels. PhD thesis, Louisiana Tech University, 1997.

[47] T. M. Segin, B. S. Tilley, and L. Kondic. On undercompressive shocks and flooding in countercurrent two-layer flows. Journal of Fluid Mechanics, 532: 217-242, 2005.

[48] Jacopo Seiwert, Christophe Clanet, and David Quéré. Coating of a textured solid. Journal of Fluid Mechanics, 669:55-63, 2011.

[49] Sidney A. Self. Focusing of spherical Gaussian beams. Applied Optics, 22(5): 658-661, 1983. 
[50] E. I. Shen and K.S. Udell. A finite element study of low Reynolds number two-phase flow in cylindrical tubes. Journal of Applied Mechanics, 52:253-256, 1985.

[51] J. H. Snoeijer, J. Ziegler, B. Andreotti, M. Fermigier, and J. Eggers. Thick films of viscous fluid coating a plate withdrawn from a liquid reservoir. Physicsal Review Letters, 100:244502, 2008.

[52] Jacco H. Snoeijer. Free-surface flows with large slopes: Beyond lubrication theory. Physics of Fluids, 18:021701, 2006.

[53] Jacco H. Snoeijer and Bruno Andreotti. A microscopic view on contact angle selection. Physics of Fluids, 20:057101, 2008.

[54] Jacco H. Snoeijer and Bruno Andreotti. Moving contact lines: Scales, regimes, and dynamical transitions. Annu. Rev. Fluid Mech., 45:269-292, 2013.

[55] Jacco H. Snoeijer and Jens Eggers. Asymptotic analysis of the dewetting rim. Physical Review E, 82:056314, 2010.

[56] Jacco H. Snoeijer, Giles Delon, Marc Fermigier, and Bruno Andreotti. Avoided critical behavior in dynamically forced wetting. Physical Review Letters, 96: $174504-1-174504-4,2006$.

[57] Kristine Spildo and Jill S. Buckley. Uniform and mixed wetting in square capillaries. Petroleum Science and Engineering, 24:145-154, 1999.

[58] Jeanman Sur, Andrea L. Bertozzi, and Robert P. Behringer. Reverse undercompressive shock structures in driven thin film flow. Physical Review Letters, 90 (12):126105, 2003. 
[59] Tomoji Takamasa and Kenji Kobayashi. Measuring interfacial waves on film flowing down tube inner wall using laser focus displacement meter. International Journal of Multiphase Flow, 26:1493-1507, 2000.

[60] G. I. Taylor. Deposition of a viscous fluid on the wall of a tube. Journal of Fluid Mechanics, 10:161, 1961.

[61] Gary Francis Teletzke. Thin Liquid Films: Molecular Theory and Hydrodynamic Implications. PhD thesis, University of Minnesota, 1983. 
APPENDICES 

A. Documentation is for figure 1.3 . 
Copyright

Clearance

Center

Confirmation Number: 11168657

Order Date: 03/19/2014

Customer Information

Customer: David Deisenroth

Account Number: 3000748323

Organization: David Deisenroth

Email: dcdeisen@mtu.edu

Phone: + 1 (906)2022180

Payment Method: Invoice

\section{This not an invoice}

Order Details

International journal of multiphase flow

Billing Status:

N/A

Permission Status: $\nabla$ Granted

Permission type: Republish or display content

Type of use: reuse in a thesis/dissertation Order License Id: 3352720790250

Number of pages $\quad 10$

Portion

figures/tables /illustrations

Number of

figures/tables

/illustrations

Format

Are you the author of

this Elsevier article?

Will you be

translating?

Title of your

thesis/dissertation

Expected completion

date

Estimated size

(number of pages)

Elsevier VAT number

Permissions price

VAT/Local Sales Tax
1

both print and electronic

No

No

Thickness measurement

of dynamic thin liquid

films generated by

plug-annular flow in

non-wetting

microchannels

May 2014

130

GB 494627212

0.00 USD

0.00 USD / 0.00 GBP

Note: This item was invoiced separately through our RightsLink service. More info 


\section{B. PrairieView Correspondence}




\section{Email Correspondence}

First of all I want to be sure you have the Harmonic timing mode selected, since that is required to run at the faster frame rates. Beyond that, it might be that the SFC controller is having difficulty finding an appropriate galvo waveform with the requested Peak-to-Peak voltage and duty cycle, or that it is simply taking so long to calculate the waveform that the software times out waiting for a response. Regardless, you will not be able to hit 2000 frames per second with the millisecond based Exposure Time field, so I would advise typing in a command manually to bypass these settings.

First, choose an exposure time that works (e.g. $3 \mathrm{~ms}$ ) without throwing the error. Then, go to the Terminal tab on the SFC window and enter the following command:

$\mathrm{Pb}$

This will respond with the current scan setting values. You can use these values in another command where you specify a different galvo frequency. For example, if $\mathrm{Pb}$ returned the following:

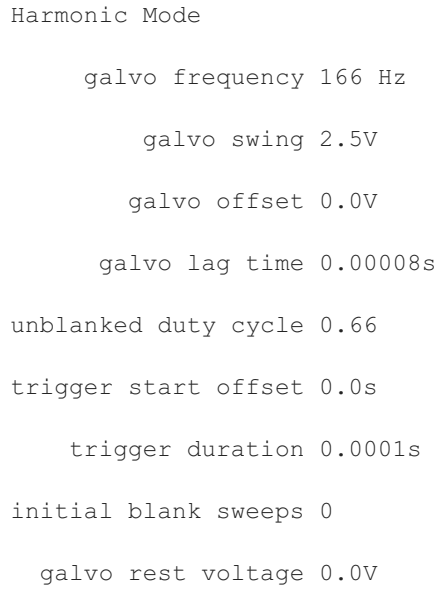

This will configure a similar scan setting, except that it runs the galvo at $1 \mathrm{kHz}$ (giving you 2000 frames per second). You can examine the new settings with the same $\mathrm{Pb}$ command.

The firmware limits you to $1500 \mathrm{~Hz}$ maximum frequency, but I would approach that value slowly to make sure the galvo is capable of doing it at the configured Peak to Peak voltage and duty cycle. If the controller has trouble moving the galvo at the desired frequency, try increasing the duty cycle (this will relax the requirements on the galvo waveform at the expense of having a less uniform illumination time across the field of view). 
I have attached an excerpt from the SFC scan control reference for the Al command so you can see what the command syntax and parameters are (I also included the full reference file in case you do not have a copy). You may need to play with the trigger offset and duration values to trigger your camera at the appropriate time during each frame. I believe Prairie View was written so that the trigger signal had a rising edge synchronized with the signal that turns on the laser (which would be somewhere around 4 to 10 microseconds before the illumination reached full intensity, depending on the response time of your particular AOTF and the speed of the DAC chip in the SFC scan control box). I believe the default trigger offset of 0 would produce a rising edge representing an end-of-frame trigger.

Also, whenever you change your laser or channel settings you may need to run it slowly with Priarie View's native controls first to make sure the laser blanking sequence is configured correctly.

I hope this is helpful, please feel free to contact me with any other questions.

Dan Daugherty

Software Engineer

Prairie Technologies, Inc.

3030 Laura Lane, Suite 140

Middleton, WI 53562-0677

Phone: 608-662-0022 x150

Fax: 608-662-0023

Email: ddaugherty@prairie-technologies.com 


\section{Galvonometer Settings}

The next figure shows typical galvo, blanking, and output trigger waveforms for a harmonic mode (the piezos do not move). In harmonic modes the galvo frequency is directly specified and a trigger output occurs every galvo half period after the start of the blanking interval (or before, depending on the trigger start offset). The larger the duty cycle of the blanking waveform the larger the amount of laser light reaches the sample and the lower the amplitude of the galvo waveform that is needed to achieve the desired galvo swing during the unblanked interval. But increasing the duty cycle also increases the degree to which the scanning area is unequally illuminated due to the varying speed of the galvo.

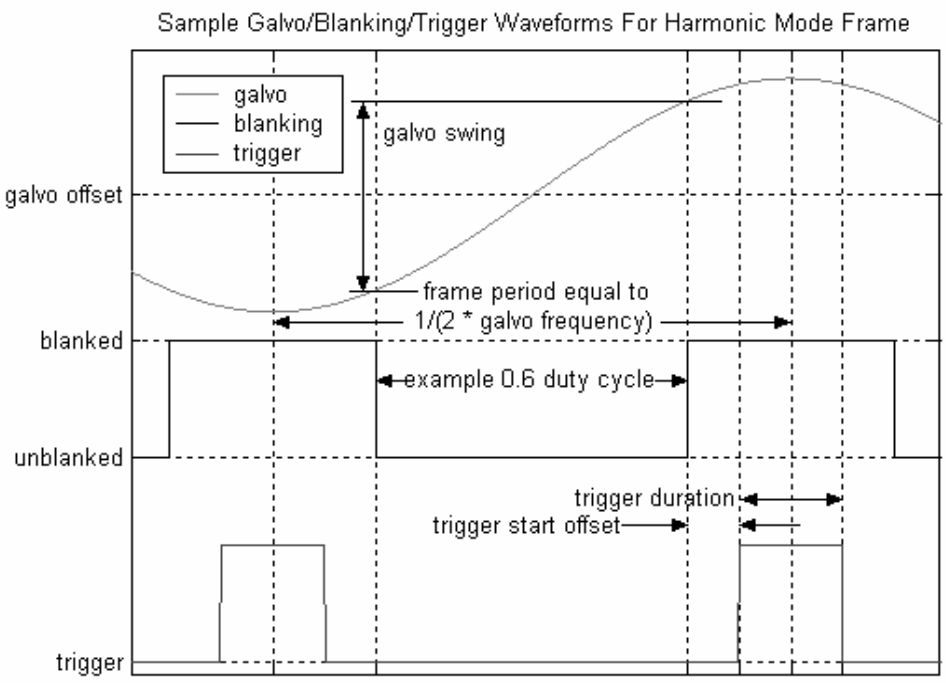

SET HARMONIC MODE SCAN PARAMETERS

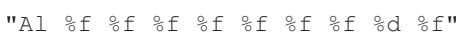

parameters:

$\# 1$ galvo frequency (allowed range is $0.001 \mathrm{~Hz}$ to $1500 \mathrm{~Hz}$ )

\#2 galvo voltage swing during unblanked portion of travel

\#3 galvo voltage offset

\#4 galvo lag time (allowed range is 0 to 0.001 seconds)

\#5 unblanked duty cycle (allowed range is 0.3 to 0.9 )

\#6 trigger start offset (up to +/- $90 \%$ of the frame period)

\#7 trigger duration (up to $90 \%$ of the frame period)

\#8 blanked sweeps (from 0-30, defaults to 0 if omitted)

\#9 galvo rest voltage (defaults to starting voltage if omitted)

example:

"Al $10001.20 .00008 \quad 5 \quad 0.0001 \quad 0 \quad-2.0 "$

returns:

"으" 
A string is returned describing the first error encountered (if any) while processing the parameters.

description:

In this scan mode the piezos are stationary while the galvo control voltage is a sinusoidal waveform. The (slit scanning) frame rate is twice the galvo frequency. The galvo control voltage follows the formula:

$\mathrm{V}(\mathrm{t})=($ galvo offset) $-\mathrm{A} * \cos \{2 * \mathrm{p} i *($ galvo frequency) $*[t+($ galvo lag) $]\}$

'A' is the amplitude of the galvo waveform which depends on the galvo voltage swing and the unblanked duty cycle. As the unblanked duty cycle decreases the galvo amplitude must increase such that the distance the galvo travels during the unblanked interval (specified by parameter \#2) remains the same. The galvo control waveform amplitude ' $A$ ' is found according to the formula:

$A=$ (galvo voltage swing) / $\{2 * \sin [$ (unblanked duty cycle)*pi/2] $\}$

The speed of galvo travel varies during the frame which causes the center of the image (where the galvo travels fastest) to be dimmer than the edges of the image according to the formula:

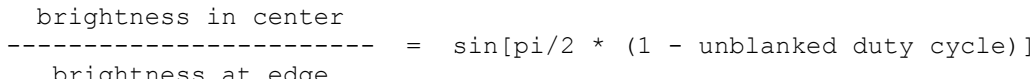

The lower the unblanked duty cycle, the more even the illumination but the less average laser power reaches the sample and the larger the galvo waveform amplitude. Since the range of travel for the galvo control voltage is $+/-5 \mathrm{~V}$, care must be taken not to use so small a duty cycle that the galvo control voltage exceeds this range. Care must also be taken to avoid large galvo control waveform amplitudes at fast frame rates, which can cause the galvo command voltage to exceed the slew rate limit on the galvo input leading to galvo malfunction.

The blanking sequence and blanking lag time are set by the 'Cu' and 'La' commands, respectively (see below). At the start of the blanked portion of each half-cycle of the galvo waveform the TRIG 1 OUT output produces a positive trigger pulse. The start of the pulse may be offset in time from from this point with the trigger start offset parameter. The duration of the pulse is specified by the trigger duration, which must be less than half the the galvo period.

Before scanning begins and after scanning ends, the trigger output is low, the AOTF lines specified with the ' $\mathrm{Cu}$ ' command are blanked, and the galvo voltage is set to the galvo rest voltage (this to park the beam away from the sample when the laser power is high enough that significant power still makes it through the AOTF while blanked). If a galvo rest voltage is not specified, then the galvo will park itself such that it will be at its starting location when the next start scan command is received.

The 'blanked sweeps' parameter specifies how many galvo half periods pass when scanning is started before normal laser unblanking and trigger outputs commence. This parameter has a maximum allowed value of 30 sweeps and a minimum of zero. 

C. Intensity Data 


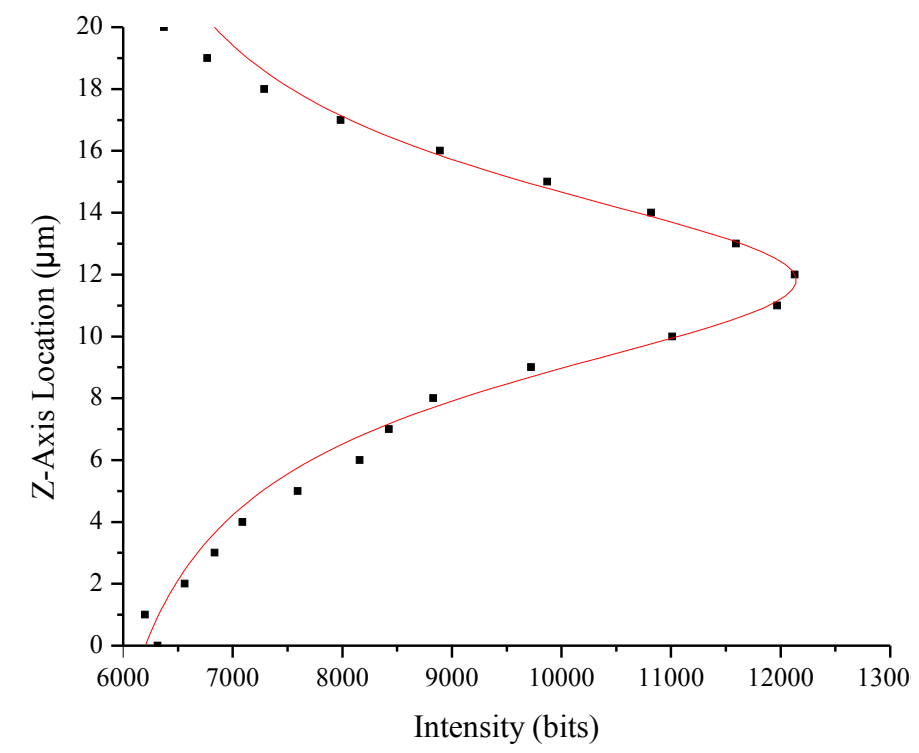

Figure 3.1. Reflection intensity of channel wall-air interface as a function of z-axis location for test runs with $C a=0.00419$

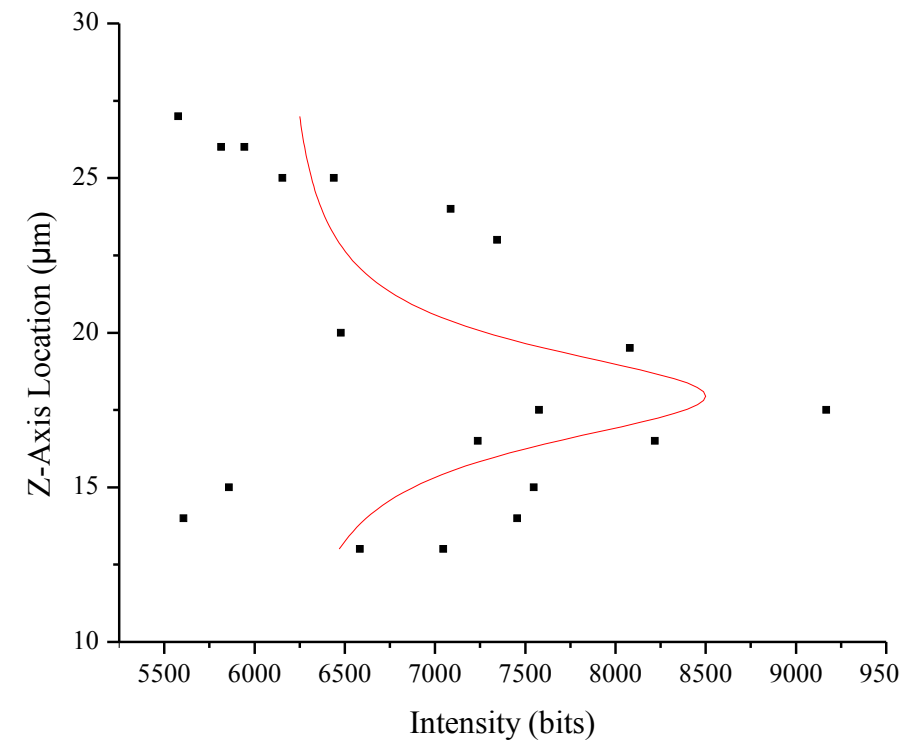

Figure 3.2. Lorentzian fit of reflection intensity in vicinity of thin film-air interface, $C a=0.00419$ 


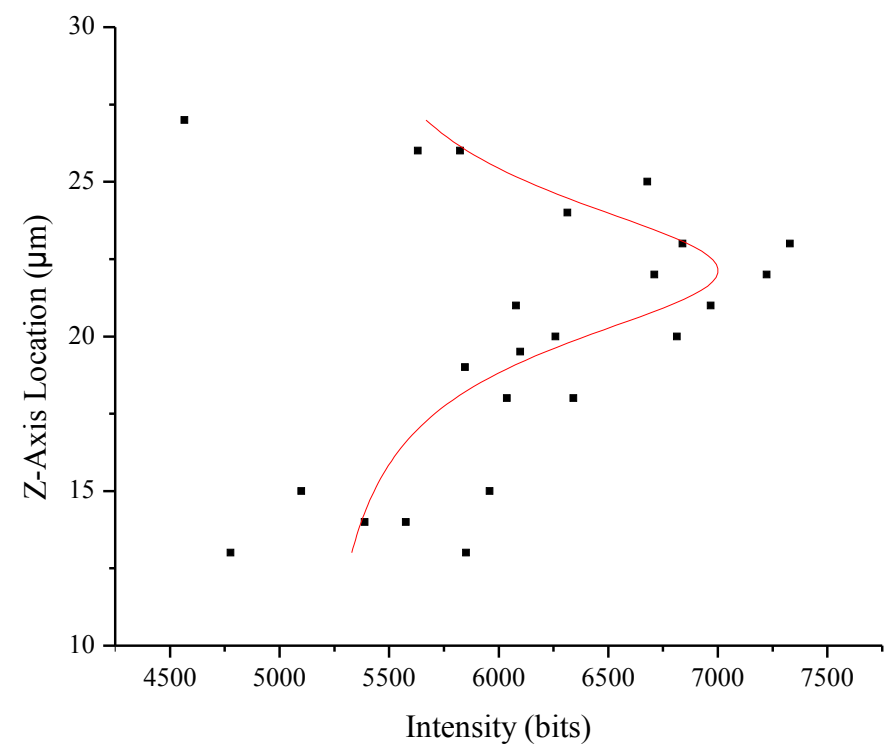

Figure 3.3. Lorentzian fit of reflection intensity in vicinity of thick film-air interface, $C a=0.00413$

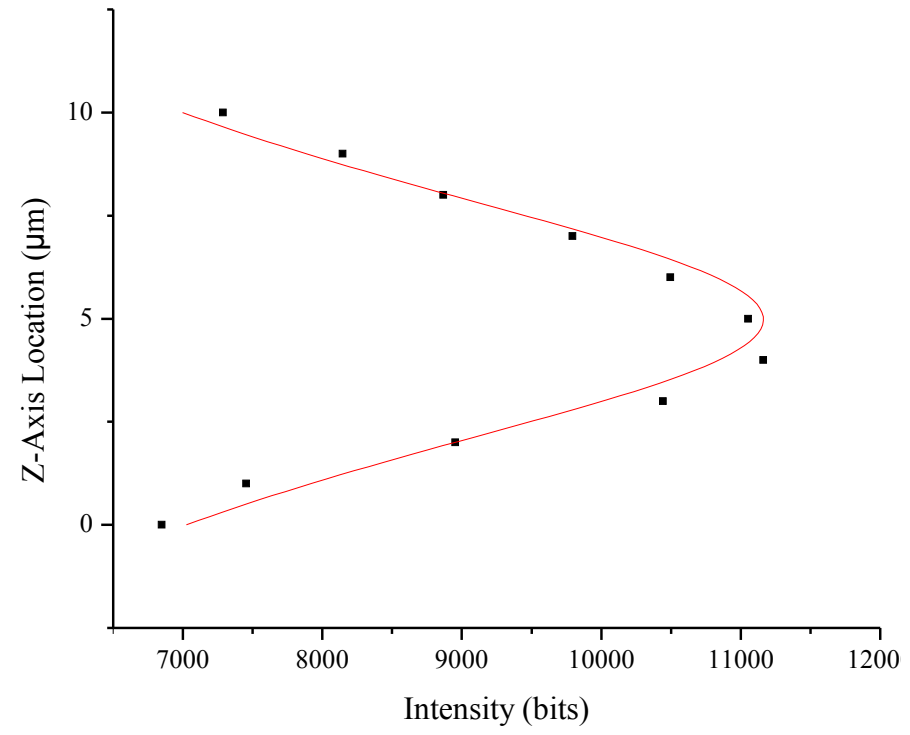

Figure 3.4. Reflection intensity of channel wall-air interface as a function of z-axis location for test runs with $C a=0.01015$ 


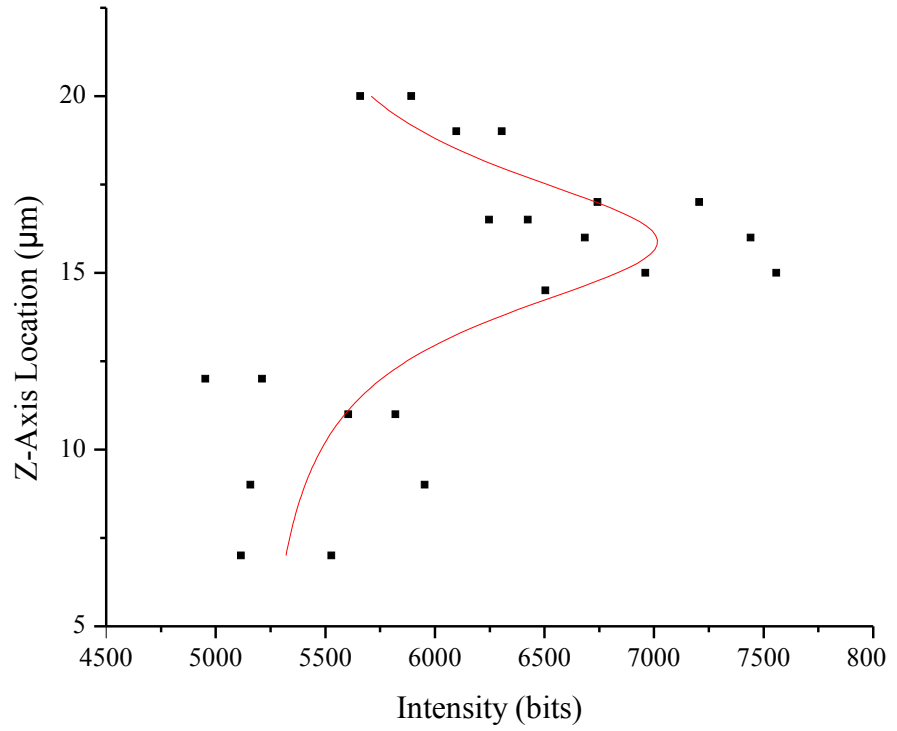

Figure 3.5. Lorentzian fit of reflection intensity in vicinity of thin film-air interface, $C a=0.01015$ 